\title{
The Major Transitions in Evolution
}

\author{
Roberta May Fisher
}

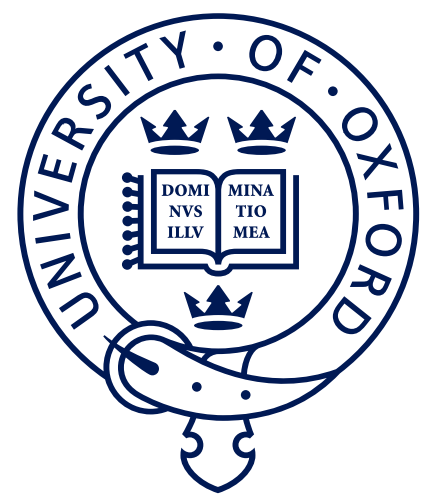

A thesis submitted for the degree of Doctor of Philosophy

Hilary term 2015 


\section{Declaration}

I declare that this thesis was composed by myself and that the work contained herein is my own except where explicitly stated in the text. This work has not been submitted for any degree or professional qualification except as specified.

Roberta Fisher, Hilary term 2015 


\section{Acknowledgements}

Firstly, I want to thank my collaborators and all the people who have given me feedback on my work: Tom Bell, Andy Gardner, Ashleigh Griffin, Kevin Foster, Charlie Cornwallis, Toby Kiers, Melanie Ghoul, Shana Caro, Philip Downing, Micheal Bentley, Nick Davies, Steffi Kapsetaki, Catherine Sheard, Lee Henry and Koos Boomsma.

Thank you to my amazing office mates (Mel, Rene, Kat, Nick, Chucky and JB) and the entire West/Griffin/Foster/MacLean lab group. A particular shout out goes to Melanie Ghoul for putting up with me the longest, and John Bruce for supplying endless photos of puppies.

Izzy, Vicki, Ellie B, Catherine, Dorothea, Danica, Klara and Ellie W (the lunch girls!), thank you for your constant friendship and wonderful distractions. Vicki, I could not have done this without you. You've been an amazing support through this DPhil and your friendship has kept me going through the hardest times.

Philip, there are too few words and too many things to say. You have been such a huge part of my DPhil, both academically and personally, and it definitely wouldn't have been the same without you. Thank you for all of the wonderful tea.

I can't not mention the two people who made it possible for me to come to Oxford back in 2007 as an undergraduate, Angela Brueggemann and Tommaso Pizzari. I will forever be grateful for the life-changing opportunity you gave me. Thank you.

And finally Stu, thank you for inspiring me to pursue evolutionary biology, allowing me to explore my ideas, giving me endless support and encouragement and providing the space for me to say crazy things. I have been incredibly lucky to work with you and learn from you, and I couldn't have asked for a better supervisor.

Thank you to Linacre College and to NERC for funding this work.

I dedicate this thesis to the two people I cherish most in the world, my fellow musketeers, Paula and Kellan Fisher. 


\section{Contributions and Publications}

I have included my contribution to the sections of each chapter in brackets, given as a percent contribution for each element.

The following published paper arose from this thesis, and is presented in Chapter 2. The paper in its published format is included in Appendix 1. Stuart West and I conceived the idea for the paper $(50 \%)$. I collected the data $(100 \%)$, made the figures $(100 \%)$ and performed the main analyses $(80 \%)$. Charlie Cornwallis performed the supplementary statistical analyses. Stuart West, Charlie Cornwallis and I wrote the paper together (33\%).

- Fisher, R. M., Cornwallis, C. K., \& West, S. A. (2013). Group formation, relatedness, and the evolution of multicellularity. Current Biology, 23(12), 11201125.

Chapter 3 is my own work, supervised by Stuart West and Tom Bell. I conceived of the experimental design with Tom Bell and Stuart West (33\%), performed the lab work $(100 \%)$ and statistical analysis $(100 \%)$, and I wrote the manuscript with Stuart West (50\%). Stefania Kapsetaki contributed to the maintenance of algal and protist cultures in the lab.

Chapter 4 is my own work, supervised by Stuart West. I collected all the data (100\%), performed the statistical analyses $(100 \%)$ and wrote the manuscript $(100 \%)$. Charlie Cornwallis and Philip Downing contributed to the statistical analysis and Lee Henry contributed to the dataset. 
I contributed to one other paper, currently in review at $P N A S$, which is included in Appendix 2. My total contribution to this paper is $25 \%$.

- West, S.A., Fisher, R.M., Gardner, A. \& Kiers, E.T. PNAS, The major evolutionary transitions in individuality. 


\section{Abstract}

The history of life has involved several major evolutionary transitions that have each led to the emergence of a new individual. Examples of major transitions in individuality include the evolution of the eukaryotic cell, multicellular organisms and eusocial societies. In each of these events, previously independently replicating units (cells, individuals etc.) cooperate to form a new individual, which can then only replicate as a whole. For this to occur, conflict between individuals needs to be minimised, to allow maximising their inclusive fitness to be roughly equivalent to maximising group fitness. It has been predicted that the way in which social groups form should be key for eliminating conflict between individuals and promoting cooperation. In this thesis, I have focused on two major evolutionary transitions; the evolution of multicellularity and the evolution of symbiosis, and show that the mode of group formation (whether groups are parentoffspring associations or not) is crucial for understanding when and why major transitions occur. Firstly, I show that the major transition to obligate multicellularity has only occurred with clonal group formation (where cells remain together after division). Secondly, I use an experimental system to show that predation pressure may be key in promoting the formation of multicellular groups in algae. Finally, I show that the mode of group formation is also important in between-species transitions. I use the evolution of symbiosis to show that transmission route of symbionts and environmental factors, determine how cooperative symbionts will be towards their hosts. 


\section{Contents}

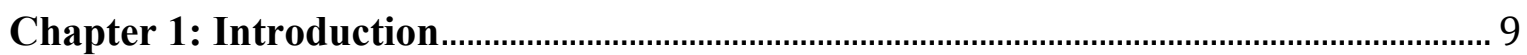

Chapter 2: Group formation, relatedness and the evolution of multicellularity ..........17

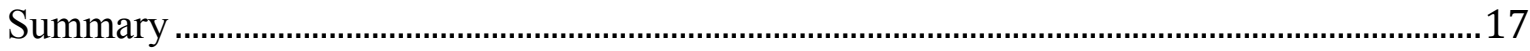

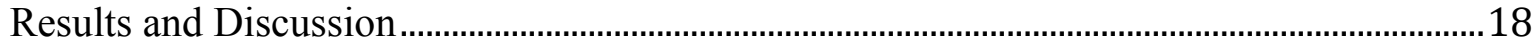

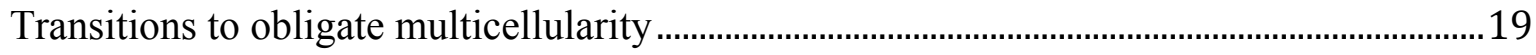

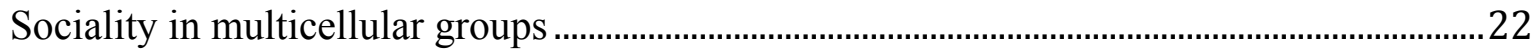

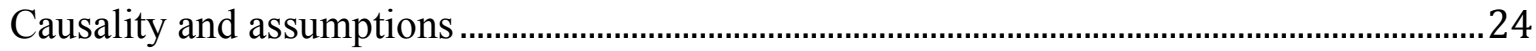

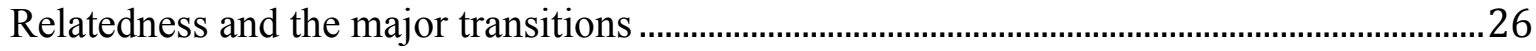

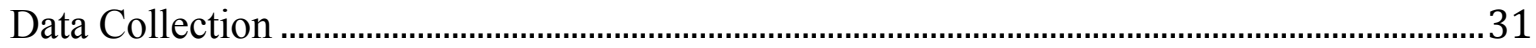

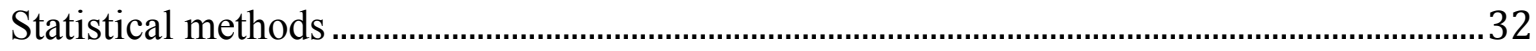

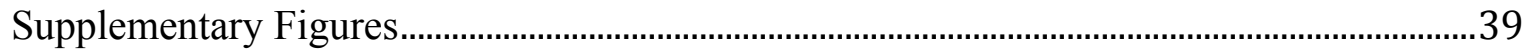

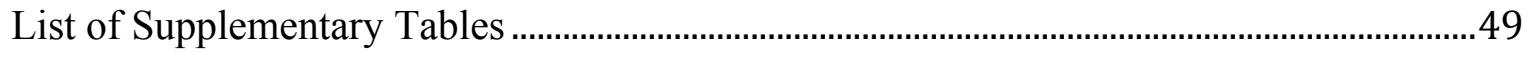

Chapter 3: Multicellular group formation in Chlorella vulgaris .......................................56

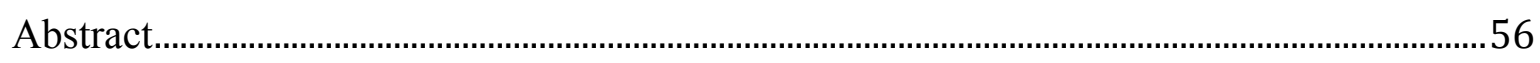

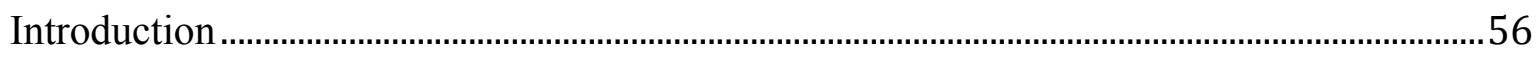

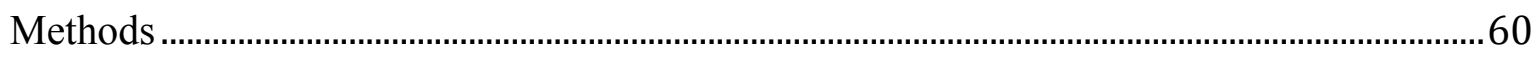

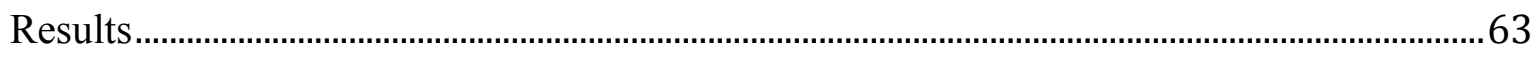

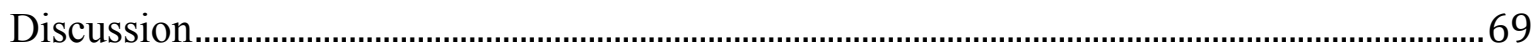

Chapter 4: The evolution of bacterial symbiosis ................................................................ 72

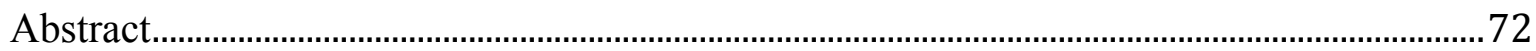

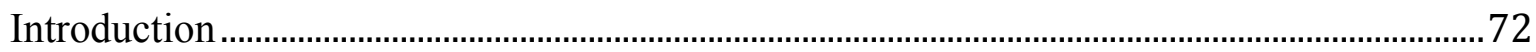

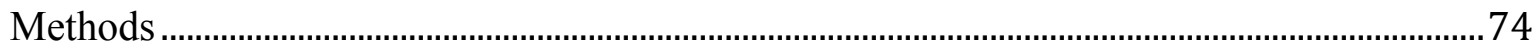

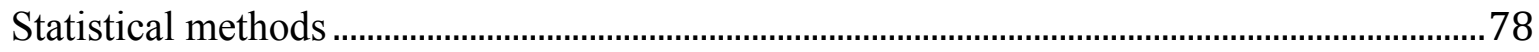

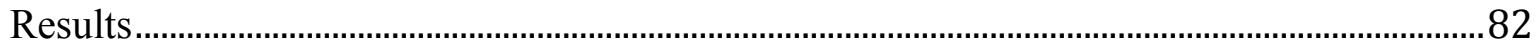

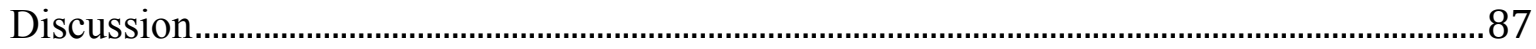

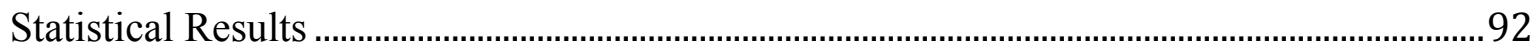


Supplementary Methods

Tree creation

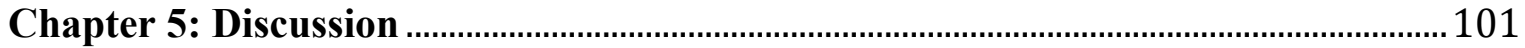

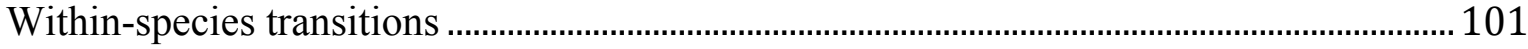

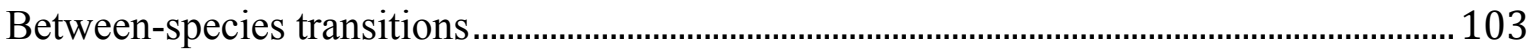

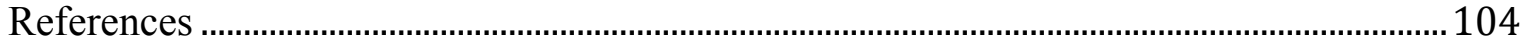

Appendix 1: Group formation, relatedness and the evolution of multicellularity

Appendix 2: The major evolutionary transitions in individuality. 


\section{Chapter 1: Introduction}

Twenty years ago, Maynard Smith and Szathmary (1995) proposed that the history of life has involved several major transitions in evolution; for example genes come together to form genomes, cells come together to form multicellular organisms and individuals form eusocial societies (Table 1). The Major Transitions framework has been the most successful attempt to unify the principles of biological organization at all levels, from gene to cell to society (Bourke 2011; Maynard Smith \& Szathmáry 1995).

Maynard Smith and Szathmary define major transitions as events that involved a change in how information is transmitted between generations, resulting in the emergence of a new individual (Maynard Smith \& Szathmáry 1995). The problem with this definition is that whilst some of the examples that they define as major transitions do involve a change in the way information is transmitted (e.g. the evolution of sex and of the genome), several others do not (e.g. the evolution of eusociality and multicellularity).

Table 1: The major transitions in evolution, as defined by Maynard Smith \& Szathmary (1995).

\begin{tabular}{lll}
\hline 1) Replicating molecules & $\rightarrow$ & Populations of molecules \\
\hline 2) Independent replicators & $\rightarrow$ & Chromosomes \\
\hline 3) RNA & $\rightarrow$ & DNA \\
\hline 4) Prokaryotes & $\rightarrow$ & Eukaryotes \\
\hline 5) Asexual clones & $\rightarrow$ & Sexual clones \\
\hline 6) Protists & $\rightarrow$ & Animals, plants, fungi \\
\hline 7) Solitary individuals & $\rightarrow$ & Colonies \\
\hline 8) Primate societies & $\rightarrow$ & Human societies \\
\hline
\end{tabular}

Major transitions in individuality, a subset of these transitions, are defined as a group of individuals joining to form a new, more complex life form that can only replicate as a whole, and where there is negligible within-group conflict (Queller 2000). In this thesis I 
only focus on major evolutionary transitions in individuality (Fig. 1), as they lead to the evolution of a new individual.

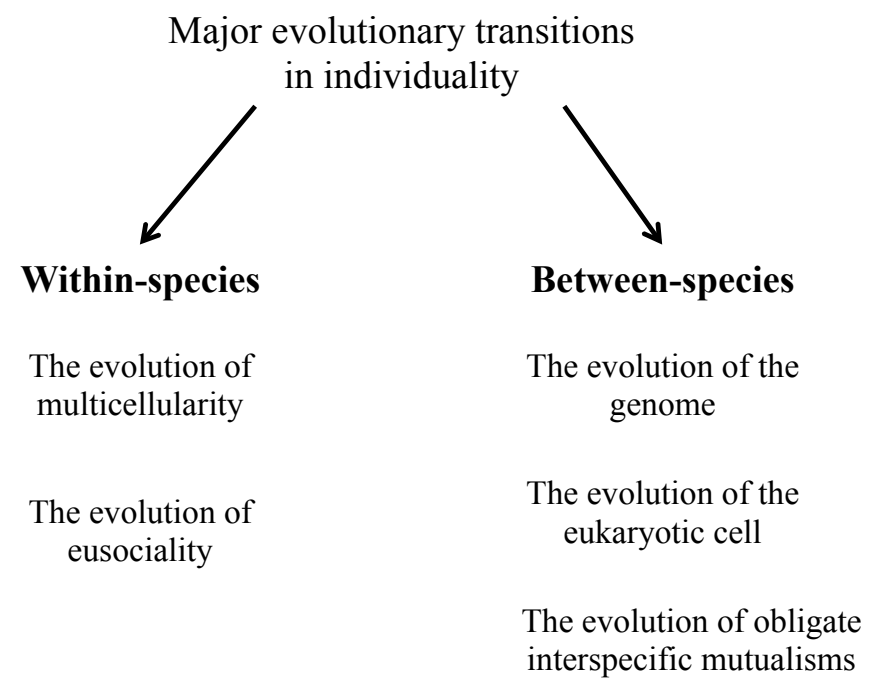

Figure 1: The major evolutionary transitions in individuality. In this figure I use the terms 'within-' and 'between-species' to refer to whether similar or dissimilar units were involved in the transition. I have eliminated the transition from primate to human societies proposed by Maynard Smith \& Szathmary (1995), as this did not involve the evolution of a new individual. I have included the transition to interspecific mutualisms, which is theoretically analogous to the evolution of the eukaryotic cell. The evolution of the genome could be considered a within- or between- species transition, but I have included it as a potential example of a between-species transition for simplicity. Queller (2000) classified major transitions as either fraternal (between relatives, where indirect benefits are important) and egalitarian (between non-relatives, where direct benefits are important). Although this is similar to the within- and between- species classification, it focuses on the mechanism of the transition rather than the type of units involved in the transition (Bourke 2011).

All major evolutionary transitions involve cooperation between individual units (e.g. genes, cells or individuals). Cooperative behaviour is any behaviour that provides a benefit to another individual, and has evolved at least partially because of this benefit (West et al. 2007). The problem of explaining cooperative behaviour from an evolutionary perspective is that individuals are expected to act selfishly to promote their own personal reproduction. The key question, then, is why cooperate? Inclusive fitness theory has gone a long way to solving this problem, and specifically Hamilton's (1964) insight that cooperative behaviour can be favoured if (1) the individual gains direct benefits from 
cooperating and/or (2) the behaviour benefits other individuals that share the same genes, i.e. relatives (Hamilton 1964). Inclusive fitness is defined as the sum of an individual's direct fitness (fitness gained through their own reproduction) and their indirect fitness (fitness gained through their relatives reproduction).

Not all cooperative groups, however, are considered major transitions. There are two criteria that set major transitions apart from other social groups. Firstly, there needs to be so little conflict between interacting units (cells, individuals etc.) that there is a coincidence of fitness interests between the individual and the group. Gardner and Grafen (2009) sum up nicely that while we should typically expect individuals to maximise their inclusive fitness, we should only expect them to behave in a way that maximises group fitness when within-group conflict has been eliminated or suppressed (Gardner \& Grafen 2009). Secondly, there needs to be complete mutual dependence between individuals, so that the social group can be considered obligate. For example, Atta ants are part of an obligate eusocial group; workers cannot reproduce without the queen and vice versa. If both these conditions are met, the social group can be considered a new individual and a major evolutionary transition has occurred. Social evolution theory has led to a relatively good understanding of how these conditions can be met for within-species transitions (Bourke 2011). However, things are still much less clear for between-species transitions.

\section{Within-species major transitions}

For within-species transitions, a potential way to remove conflict over whether to reproduce or help, is if individuals are equally related to the offspring that they could help raise $\left(r_{h}\right)$ and their own offspring $\left(r_{o}\right)$. In this situation, the individual will be selected to do 
whatever maximises the production of offspring, and this then entirely depends on the benefit $(B)$ and the cost $(C)$ of the cooperative behaviour (Fig. 2). This can be achieved through the way in which social groups form (Fig. 3).

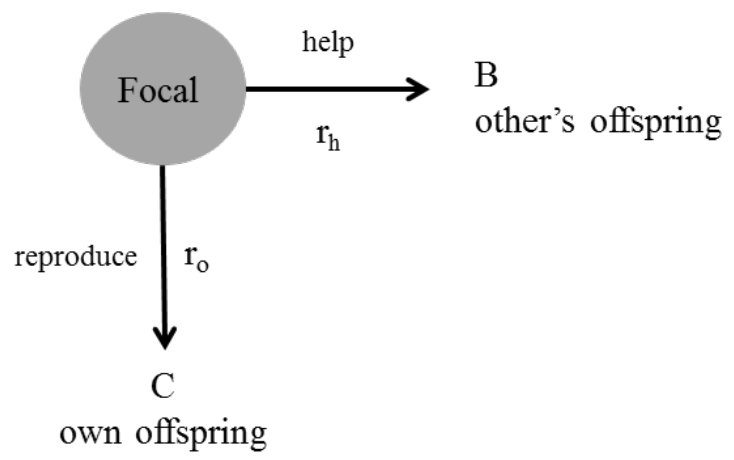

Figure 2: Relatedness and Hamilton's rule. A focal individual is related to individuals it could potentially help $r_{h}$, and is related to its own offspring $r_{o}$. B is the benefit of the behaviour to the recipient and $C$ is the cost to the focal individual. A focal individual will be favoured to help another if $r_{h} B-r_{o} C>0$.

Several comparative studies have shown that within-species major transitions have only occurred when the route of group formation has meant that $r_{h}=r_{o}$, leading to negligible conflict between individuals (Boomsma 2009; Fisher et al. 2013; Queller 2000; Hughes et al. 2008). For multicellular organisms, obligate multicellularity has only evolved where the organism is formed through cells sticking together after division. In this situation, conflict between individual cells is effectively eliminated and so, if ecological conditions allow, a major transition can occur. When cells aggregate together, there is the potential for appreciable conflict between cells, because $r_{h} \neq r_{o}$, and this has never led to a major transition to multicellularity (Chapter 2, Fisher et al. 2013). Furthermore, in eusocial societies, it has been demonstrated using phylogenetic techniques that monogamy and subsociality (where offspring stay behind to help their parents) are ancestral to all obligately eusocial clades, and so the major transition to eusociality (Hughes et al. 2008; Boomsma 2009). 
There are several good examples of within-species major transitions in individuality, such as the evolution of obligate multicellular organisms (animals, plant, fungi) and the evolution of obligate eusocial societies, for example ants and termites (Crespi \& Yanega 1995).

\section{Between-species major transitions}

Although we have a relatively good theoretical understanding of the conditions that lead to negligible conflict in within-species transitions, there has been much less attention paid to between-species transitions. As in within-species transitions, the way in which groups form may be key for reducing conflict and increasing cooperation between individual units (Bourke 2011). In the case of interspecific mutualisms, a strictly vertically transmitted symbiont can only maximise its fitness by increasing the reproductive success of the host (Herre et al. 1999; Frank 1996b, Fig. 3). For example, Buchnera bacteria that infect aphids are strictly vertically transmitted to aphid offspring, such that the fitness interests of the bacteria and the host are completely aligned (Douglas 1996). In contrast, symbionts that are transmitted horizontally between hosts, like Rhizobia bacteria, could be selected to help their hosts less than would be optimum from the host's perspective (Sachs et al. 2010; Kiers \& Denison 2008), leaving the potential for conflict over reproduction and transmission.

Although strict vertical transmission is likely to lead to the alignment of host and symbiont reproductive interests, there is a caveat to this. If symbionts have uniparental inheritance there is potential for conflict between hosts and symbionts over sex allocation. 
For example, certain symbiotic bacteria, e.g. Wolbachia, have become 'reproductive manipulators' by evolving mechanisms to bias the offspring sex ratio of the host to maximise their transmission to future generations (Bright \& Bulgheresi 2010).

The evolution of the eukaryotic cell is also an example of a between-species major transition in individuality (Appendix 1). The endosymbiotic origin of mitochondria and plastids led to the formation of a new individual, the eukaryotic cell, which could then only replicate as a whole (Keeling 2010; Cavalier-Smith 2006). Another potential between-species major transition is the symbiosis between Buchnera bacteria and their aphid hosts, as the aphids rely on Buchnera for essential amino acids, and Buchnera are not found outside of its host (Douglas 1996, Fig. 3). 


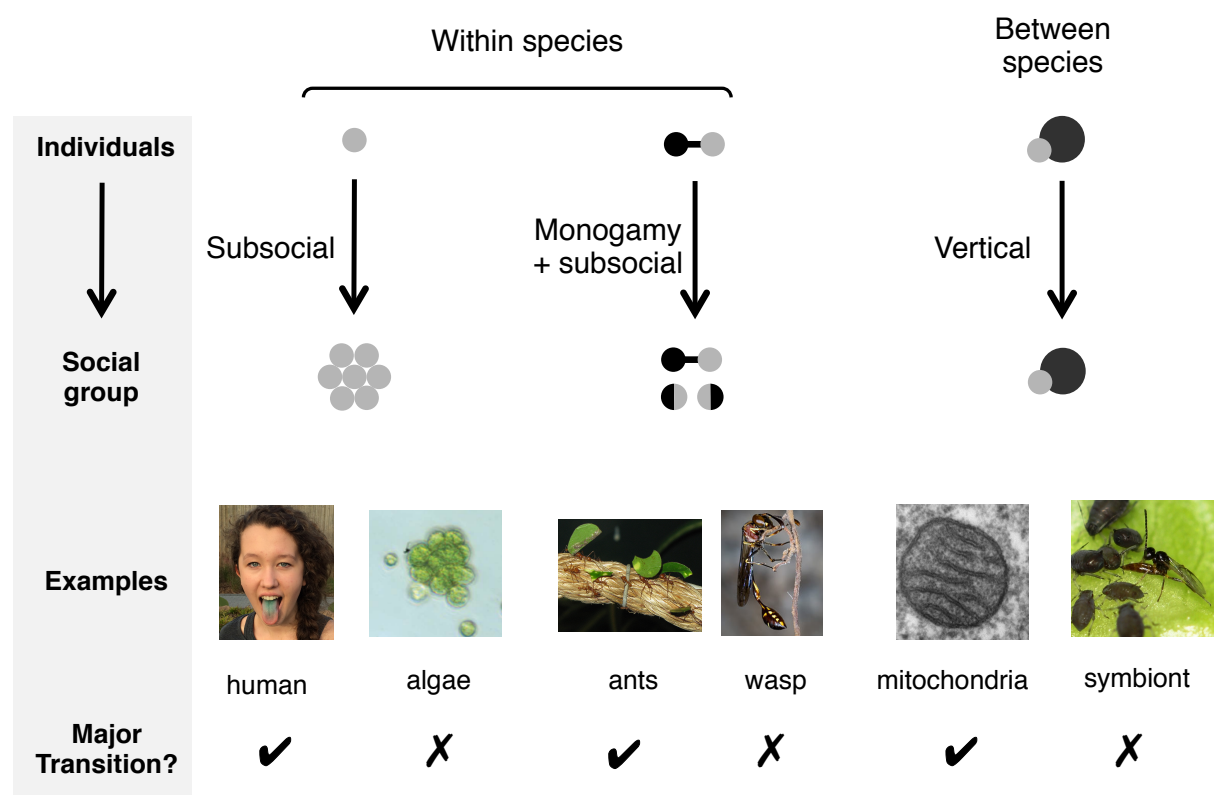

Route of social group formation leads to appreciable conflict

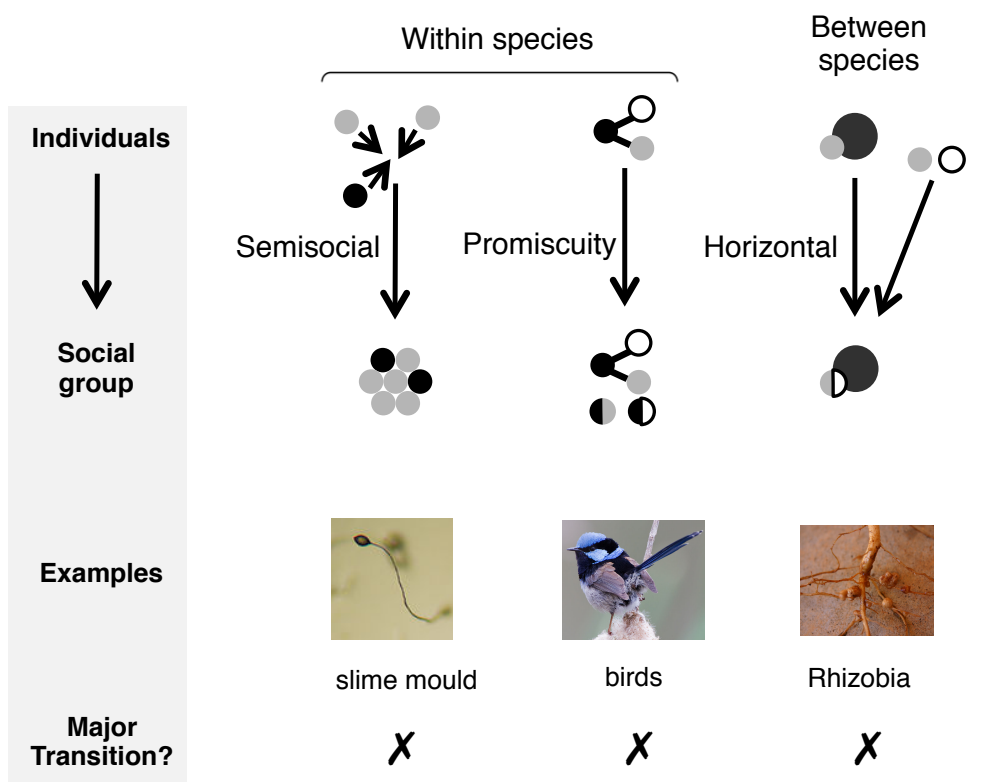

Figure 3: Group formation is a major determinant of when major transitions have taken place. The way in which groups form is a major determinant of when major transitions have taken place because it determines relatedness and the potential for within-group conflict. Within-species transitions have only taken place when offspring stay to help their parents (subsocial) and either asexual reproduction or lifetime monogamy. Between-species transitions seem to involve similarly restrictive group formation, such as vertical transmission leading to clonal symbionts whose interests are aligned with their hosts. Different coloured circles represent either genetically distinct individuals (within-species) or individuals of different species. Larger circles represent hosts with smaller circles representing their symbionts. The images, from left to right, show: obligately 
multicellular human, facultatively multicellular Chlamydomonas, Atta ants, Stenogastrine hover wasp, mitochondrion, Hamiltonella defensa (image shows parasitoid infecting aphid containing Hamiltonella), facultatively multicellular Dictyostelium slime mould, cooperatively breeding superb fairy wren, Rhizobia-legume mutualism.

\section{Thesis and chapter aims}

The main aim of this thesis is to identify common principles that apply across major evolutionary transitions in individuality. Specifically, I focus on mechanisms that reduce or eliminate conflict. I explore this by looking at both within- and between-species transitions and identify key underlying principles that apply to both.

Chapters 2 and 3 deal with the evolution of multicellularity; the transition from single cells to multicellular organisms. In Chapter 2, I examine how the route of social group formation influences the likelihood of a major transition to obligate multicellularity using a phylogenetically controlled comparative analysis. In Chapter 3, I explore the transition to multicellularity using the alga Chlorella vulgaris as a model experimental system, focusing on the role that predation plays in promoting social group formation in this species.

In Chapter 4, I focus on the evolution of bacterial symbiosis. I test the hypothesis that vertical transmission of symbionts should limit host-symbiont conflict and lead to greater cooperation between symbionts and their hosts using a phylogenetically controlled metaanalysis.

In the Appendix 2, myself and co-authors review the Major Transitions framework. 


\section{Chapter 2: Group formation, relatedness and the evolution of multicellularity*}

\section{Summary}

The evolution of multicellular organisms represents one of approximately eight major evolutionary transitions that have occurred on earth (Leigh 1995; Maynard Smith \& Szathmáry 1995; Queller 2000; Bourke 2011). The major challenge raised by this transition is to explain why single cells should join together and become mutually dependent, in a way that leads to a more complex multicellular life form that can only replicate as a whole. It has been argued that a high genetic relatedness $(r)$ between cells played a pivotal role in the evolutionary transition from single-celled to multicellular organisms, because it leads to reduced conflict and an alignment of interests between cells (Heath-Heckman et al. 2013; Maynard Smith \& Szathmáry 1995; Queller 2000; Strassmann \& Queller 2010; Michod \& Roze 2001; Boomsma 2013, 2007, 2009; Grosberg \& Strathmann 1998, 2007; Kuzdzal-Fick et al. 2011; Gardner \& Grafen 2009; Michod 1997; Keller 1999; Frank 1995; Hamilton 1964). We tested this hypothesis with a comparative study, comparing the form of multicellularity in species where groups are clonal $(r=1)$, to species where groups are potentially non-clonal $(r \leq 1)$. We found that species with clonal group formation were more likely to have undergone the major evolutionary transition to obligate multicellularity, had more cell types, a higher likelihood of sterile cells and a trend towards higher numbers of cells in a group. More generally, our results unify the role of group formation and genetic relatedness across multiple

\footnotetext{
* Published as: Fisher, R M., C K. Cornwallis, and S A. West. "Group formation, relatedness, and the evolution of multicellularity." Current Biology 23.12 (2013): 11201125 (appended).
} 
evolutionary transitions, and provide an unmistakable footprint of how natural selection has shaped the evolution of life (Leigh 1995).

\section{Results and Discussion}

The evolution of life on earth, from the simplest replicating molecules to complex animal societies has involved approximately eight major evolutionary transitions in individuality (Maynard Smith \& Szathmáry 1995; Queller 2000; Bourke 2011). In each of these transitions, a group of individuals that could previously replicate independently join together to form a new, more complex life form that can only replicate as a whole. For example, genes come together to form genomes, cells join together to form multicellular organisms, and multicellular organisms join together to form eusocial societies. The major challenge raised by each of these transitions is to explain why individuals should join together and become mutually dependent, in a way that leads to a more complex individual (Maynard Smith \& Szathmáry 1995; Queller 2000; Bourke 2011).

Here, we use a phylogenetically based (Hadfield \& Nakagawa 2010) comparative study to test how the relatedness between interacting cells influenced both the likelihood of the major evolutionary transition to obligate multicellularity, and the level of sociality in multicellular groups. We obtained data on 168 species, with representatives from all multicellular lineages, except the diatoms and Charaphyte algae (Fig. 1). We used life history data on how groups form to infer relatedness. Specifically, when groups form by cells remaining with their parents, then groups are clonal ( $r=1 ; 149$ species). This usually involves the group going through a single celled (unicellular) stage (Grosberg \& Strathmann 1998, 2007). In contrast, if groups form by cells aggregating together, then 
relatedness could be anything from zero to one $(r \leq 1)$, but is likely to be non-clonal $(r<$ 1; 19 species) (Gilbert et al. 2007).

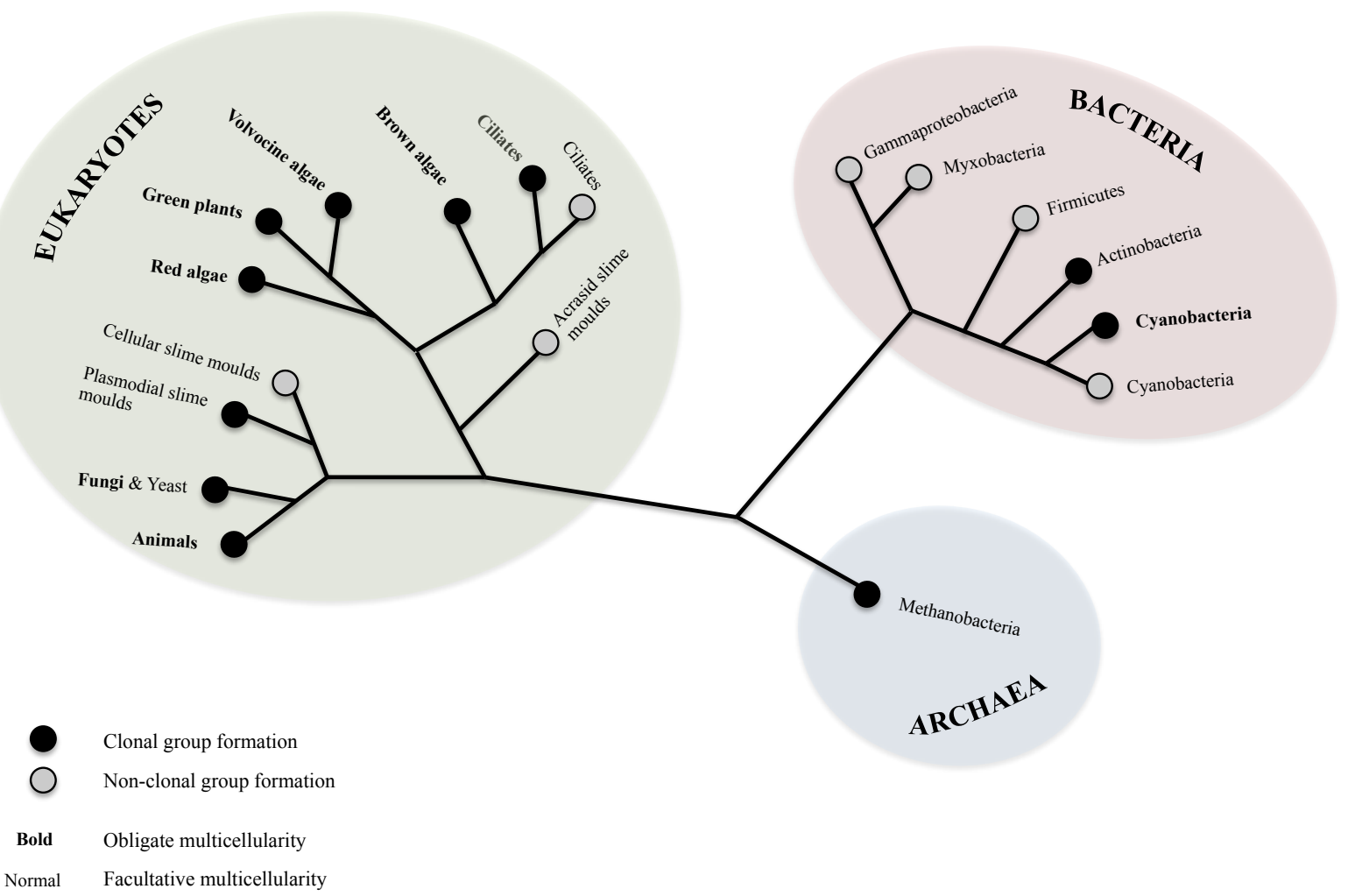

Figure 1: Multicellularity across the tree of life. The figure shows an overview phylogram of the taxa in our data set. We have labeled each taxon according to whether multicellular groups are either clonal (black circles) or non-clonal (grey circles), and whether multicellularity is an obligate (bold font) or a facultative (normal font) part of the life-cycle. The relatively low number of times that multicellularity has evolved, especially in taxa with non-clonal groups, emphasises the importance of collecting data on specifically targeted groups, to increase the statistical power of phylogenetically based analyses.

\section{Transitions to obligate multicellularity}

We first examined whether relatedness influenced the likelihood that groups underwent the transition to obligate multicellular groups. Our distinction here is between obligately multicellular species, which can only complete their life-cycle as a multicellular organism, and facultatively multicellular species, which are able to complete their life-cycle as 
unicells, and only become multicellular under certain environmental conditions. For example, Dictyostelium species can remain in their unicellular state for many generations, without the need to form a multicellular fruiting body, which they only do under certain harsh conditions, and so we classify them as facultatively multicellular. In contrast, in mammals, the unicellular stage is finite and ultimately always has to lead to the multicellular stage, and so we classify them as obligate multicellular. Consequently, we are focusing on whether one of the key requirements for a major evolutionary transition in individuality has occurred, termed contingent irreversibility (Maynard Smith \& Szathmáry 1995; Bourke 2011).

We found that species with clonal groups were significantly more likely to have made the transition to obligate multicellularity (Figs. $2 \& 3$; pMCMC $=0.0002$ ). Overall, $75 \%$ (9/12) of the lineages with clonal group formation had made the transition to obligate multicellularity, whereas the five lineages with potentially non-clonal group formation had only led to facultative multicellularity. In order for a multicellular group to have made a major transition in individuality, requires both obligate multicellularity, and that there is lack of within group conflict such that the group be thought of as an individual (Maynard Smith \& Szathmáry 1995; Queller 2000; Bourke 2011). Clonality leads to no within group conflict (Gardner \& Grafen 2009). Consequently, because obligate multicellularity has only evolved in species with clonal groups, all of these species have made a major transition in individuality. Obligate multicellularity is feasible in species with non-clonal group formation - all it would require is that cells always aggregate to complete a necessary part of their life cycle (i.e. not just under certain conditions). Given that we found no evidence for non-clonal organisms having evolved obligate multicellularity, this 
suggests that the genetic conflict that arises from lower relatedness $(r \leq 1)$ inhibits this major transition in individuality.

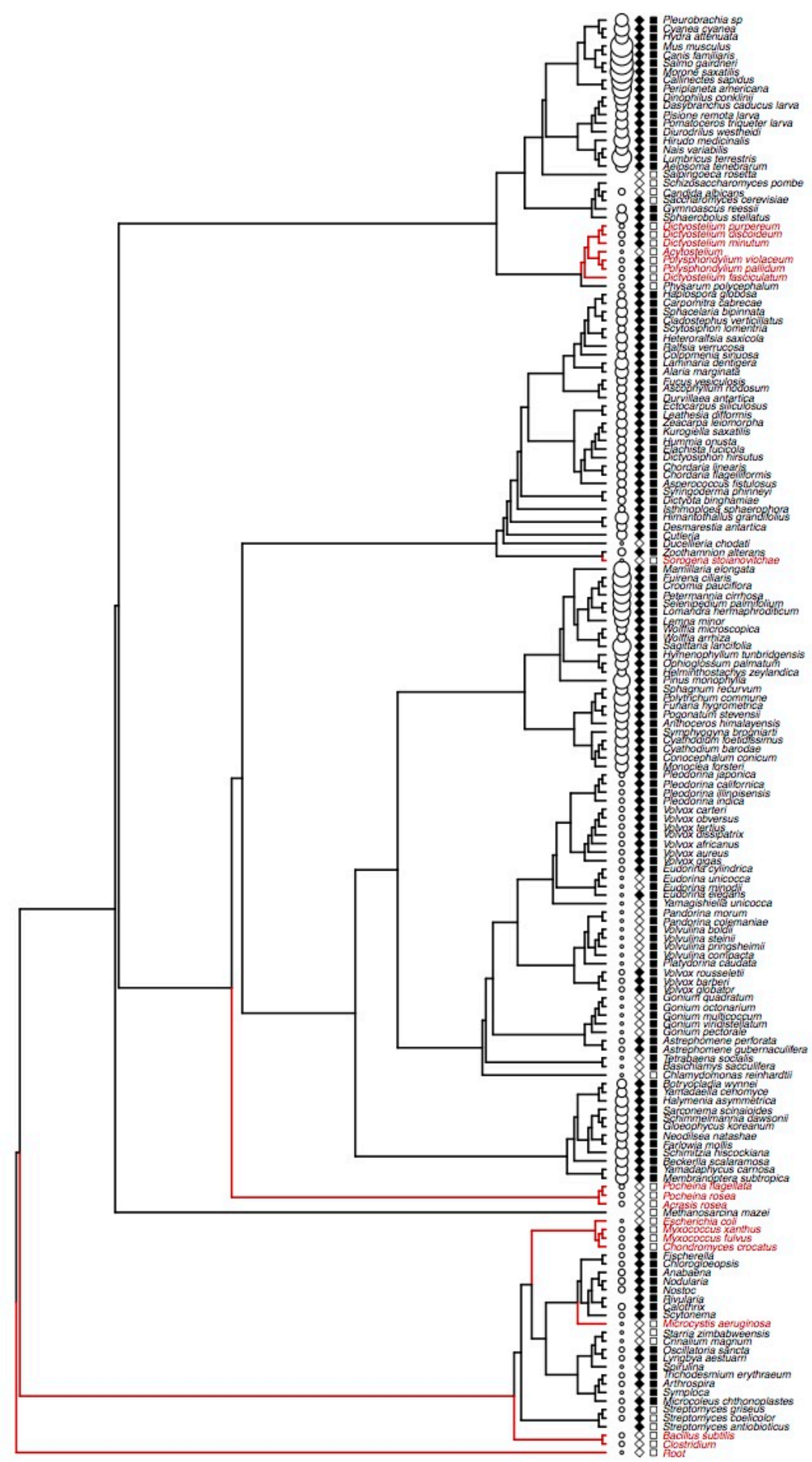

Figure 2. Evolutionary relationships between clonality and multicellular traits. The figure shows the evolutionary transitions between clonal (black tips and edges) and non-clonal (red tips and edges) group formation and its correlation with obligate (black squares) versus facultative 
multicellularity (white squares), presence of sterile soma (black diamonds = sterile soma, white diamonds $=$ no sterile soma) and the number of different cell types (white circles: size $=$ natural logarithm of number of cell types). Ancestral reconstruction of clonal and non-clonal states conducted using Bayesian phylogenetic mixed models in MCMCglmm.

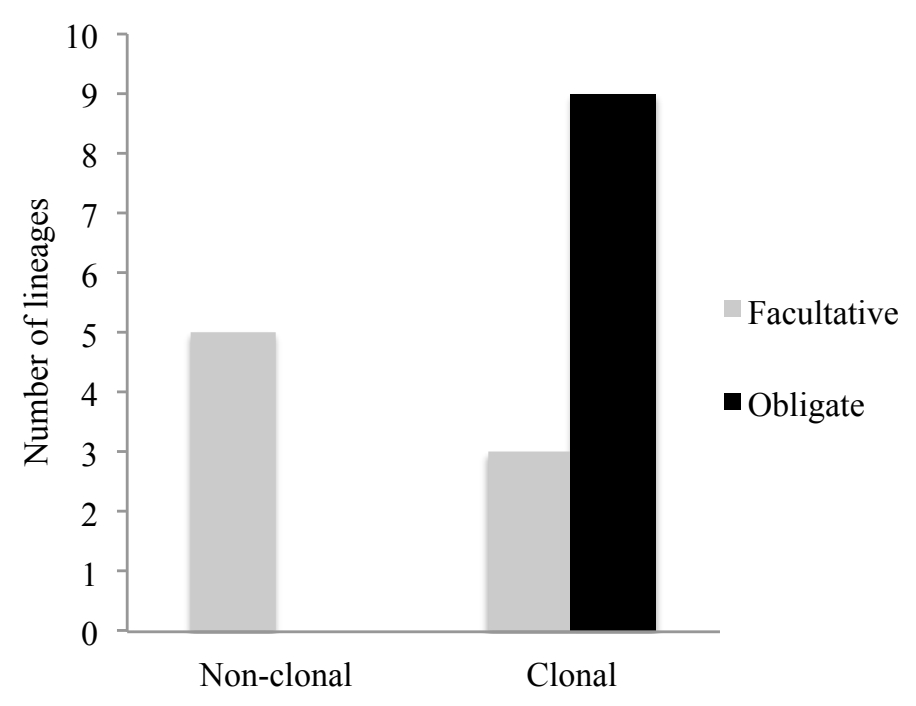

Figure 3. The evolutionary transitions to facultative and obligate multicellularity. The figure shows the number of lineages in which obligate multicellularity has evolved, comparing lineages where groups are clonal (9/12 lineages) or potentially non-clonal (0/5). Obligate multicellularity has only evolved in clonal groups.

\section{Sociality in multicellular groups}

We then examined whether relatedness influenced the level of sociality in multicellular groups. We collected data on four life history variables: the number of different cell types that can occur in each group; whether or not species had sterile cells; the percentage of the total number of cells that were sterile in species that had sterile cells; and the number of cells in the group. The number of different cell types that can occur in a group is analogous to the number of castes in eusocial insect colonies, and hence represents the extent to which different group members specialise in different roles (Bourke 2011). Sterile cells represent a case of extreme altruism, analogous to sterile workers in eusocial insects, forgoing any opportunity to reproduce directly in order to help others (Strassmann 
\& Queller 2010). The number of cells in a group is seen as one of the traits that contribute to and correlate with group complexity (Bourke 2011).

We found that species with clonal groups had significantly higher numbers of cell types, and a significantly higher probability of having sterile cells. Species with clonal groups had, on average, approximately six times as many cell types as species with non-clonal groups (Fig. $2 \& 4 a ;$ pMCMC $=0.0008$ ), and were approximately twice as likely to have sterile cells (Figs. $2 \& 4 \mathrm{~b}$; pMCMC $=0.02$ ). These significant influences of relatedness are particularly impressive given that: (a) ecological costs and benefits also matter, not just relatedness (Hamilton 1964); (b) relatedness may still be extremely high, for example $r=0.98$ (Gilbert et al. 2007) in the non-clonal groups; (c) the limited number of evolutionary transitions to multicellularity. This emphasises that not only does relatedness matter, but that it matters a lot.

We found that species with clonal groups had a higher proportion of sterile cells (in the species with sterile cells), and a greater number of cells, but that these differences were non-significant. In species with sterile cells, those that formed clonal groups had approximately three times the proportion of sterile cells in comparison to species with non-clonal groups (Fig. 4c; pMCMC = 0.41). Species with clonal groups had, on average, approximately 100 times as many cells in their group as species with non-clonal groups (Figs. $2 \& 4 \mathrm{~d}$; pMCMC $=0.27$ ). This lack of significant differences, despite their magnitude, reflects both high variability and a limited statistical power, due to the small number of independent evolutionary origins of multicellularity, especially those with potentially non-clonal group formation (Figs. $1 \& 3$ ). For example, the comparison of the proportion of sterile cells was limited primarily to a comparison of clonal Volvocine algae 
and cyanobacteria with the non-clonal cellular slime moulds (Fig. 3). The high variability may reflect that whilst a higher relatedness can favour more sterile cells, and larger groups, the ecological costs and benefits of these traits could vary across taxa.

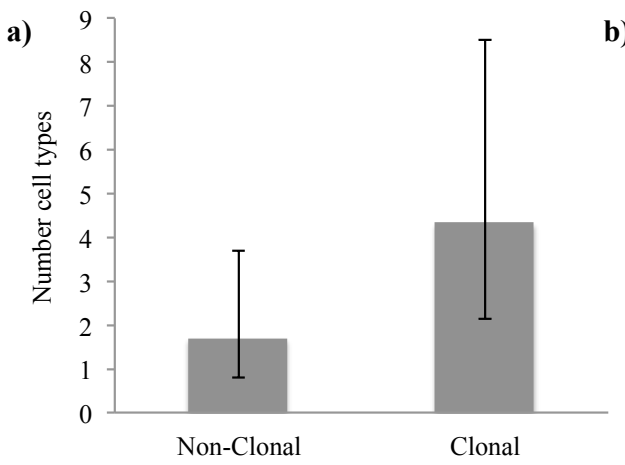

b)
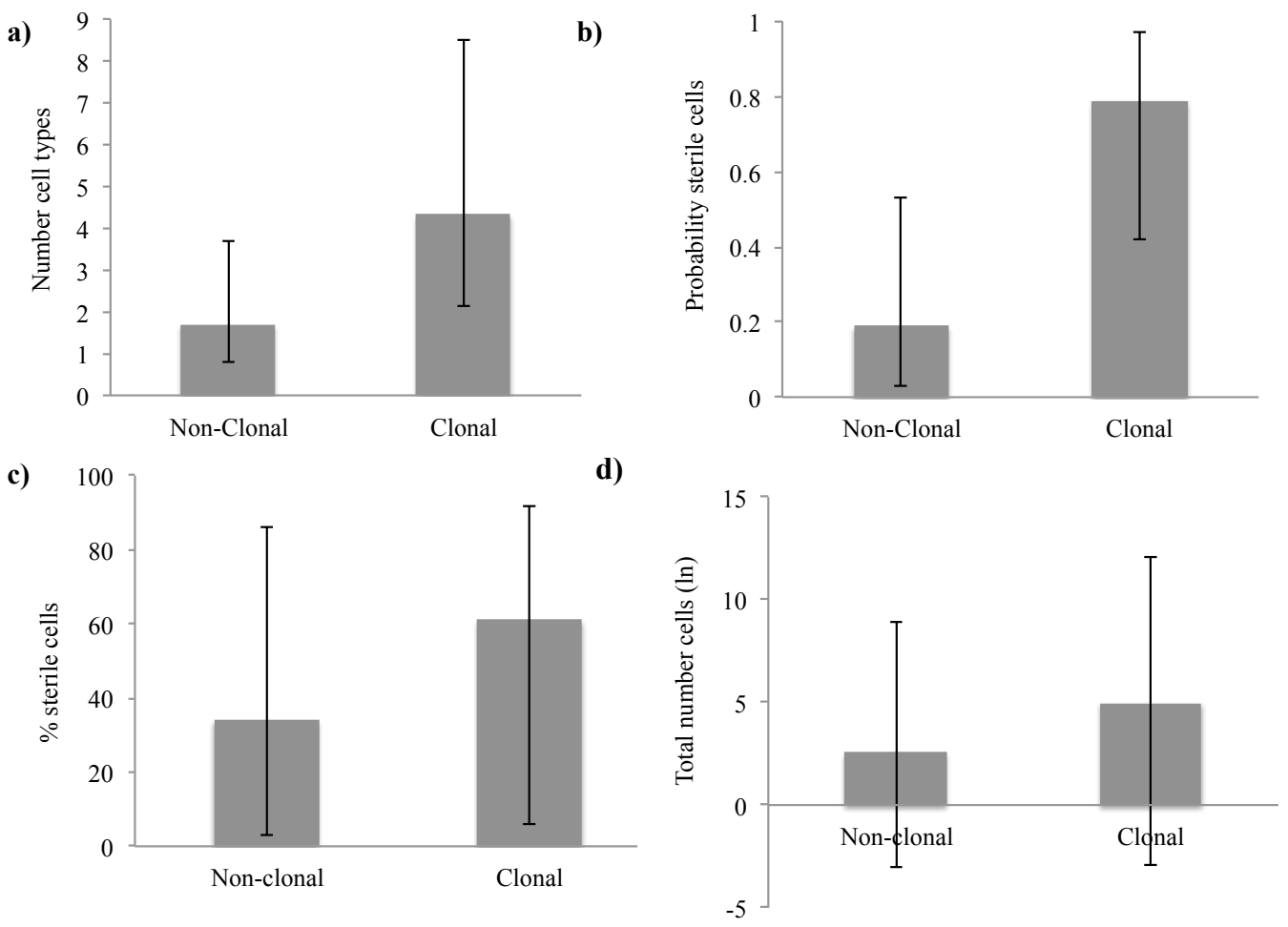

Figure 4. Sociality and the method of group formation. The graphs show, for multicellular groups that are either clonal or potentially non-clonal, the: (a) number of cell types; (b) probability of having steriles cells; (c) percentage of sterile cells in species with a sterile soma; (d) total number of cells. Estimates are posterior modes with $95 \%$ credible intervals from Bayesian phylogenetic mixed models. Number of cell types are backtransformed from a Poisson distribution with $\log$ link function, probability of sterile cells from a binary distribution with logit link function, proportion of sterile cells from a binomial distribution with a logit link function and total number of cells is on a logarithmic scale.

\section{Causality and assumptions}

We have examined separately the influence of relatedness on whether a transition to obligate multicellularity has occurred, and the different traits that determine the level of sociality, such as whether there are sterile cells in the group. Previous work has examined 
multiple traits simultaneously, by comparing simple and complex multicellular species, where complexity is defined by the presence of sterile cells, an early germ-soma split, a high number of cell types and a large number of cells (Bourke 2011; Grosberg \& Strathmann 1998, 2007). However, whilst the simple/complex distinction is likely to be correlated with whether a major transition has occurred, it is not a defining feature and the correlation can break down (Boomsma 2013). For example, Dictyostelium purpureum and Volvox carteri are both simple, having a sterile soma and the same number of cell types, but only $V$. carteri has undergone the major transition to obligate multicellularity. Furthermore, whilst complex multicellularity has only evolved in species with a single cell stage (unitary development) that leads to clonality $(r=1)$ (Grosberg \& Strathmann 1998, 2007), there is no significant correlation between relatedness and whether multicellularity is simple or complex ( $\mathrm{pMCMC}=0.21$ ). A problem here is that complex multicellularity has evolved only five times, in sister groups; animals, fungi, plants, red algae and brown algae (Bourke 2011; Grosberg \& Strathmann 2007). Consequently, complex multicellularity hasn't evolved in enough separate places on the tree of life to provide statistical power for a formal comparative analysis (Hadfield \& Nakagawa 2010; Harvey \& Purvis 1991).

Our results are robust to the assumptions that we make about how relatedness and the various social traits coevolve. Our above analyses assumed that relatedness does not evolve and causally effects each of the social traits. Whilst the causal effect of relatedness on the evolution of social traits is strongly supported (Davies et al. 2012), the variation in relatedness amongst cells may change over evolutionary time. We therefore reanalysed our data, allowing both relatedness and the social traits to evolve across the tree, whilst relaxing our assumption of causality (Felsenstein 2012). These analyses gave qualitatively identical results, with a significant statistical correlation between relatedness and obligate 
versus facultative multicellularity $(p<0.0001)$, number of cell types $(p=0.003)$ and probability of sterile soma $(\mathrm{p}=0.009)$, but not number of cells $(\mathrm{p}=0.08)$, or the proportion of sterile cells in the species that had sterile cells ( $p=0.34$; Table S3).

In almost all of the species with clonal group formation, a unicellular stage is involved, where the group (or organism) arises from a single cell, usually a zygote or spore (Grosberg \& Strathmann 1998, 2007). The exception to this is in species with multicellular propagules, when the propagules are formed by cells remaining with their parents, as in some Cyanobacteria (Schirrmeister et al. 2011). Another consequence of a unicellular stage is that it leads to a shared developmental history that could facilitate the scope for coordinated development among cells, for example into different cell types (Grosberg \& Strathmann 2007). This should not be seen however as a competing explanation to relatedness, as it is a mechanistic (proximate) and not a selective (ultimate) issue (Mayr 1961; Tinbergen 1963). For example, whilst the potential for coordination could place a limit on the number of cell types (the strategy set open to natural selection), we still need an explanation for why multiple cell types are favoured (which strategy is favoured by natural selection). Nonetheless, interactions can be important, as natural selection can only act on the options that are developmentally and mechanistically possible.

\section{Relatedness and the major transitions}

More generally, when combined with data from previous studies, our results show how relatedness provides a single life-history variable that plays a key role in explaining evolutionary transitions that involve members of the same species joining together to cooperate (Queller 2000; Bourke 2011; Boomsma 2013, 2007, 2009; Hatchwell 2009). 
We have shown how a higher relatedness between cells leads to a higher likelihood of obligate multicellular groups, a higher number of cell types and a higher likelihood of sterile cells (Figs. 2-4). A previous experimental study has shown that Dictyostelium discoideum loses cooperative fruiting body formation if kept under conditions of low relatedness (Kuzdzal-Fick et al. 2011). Previous comparative studies have shown how a higher within group relatedness is correlated with cooperative breeding in both birds and mammals (Cornwallis et al. 2010; Lukas \& Clutton-Brock 2012), and the evolution of eusociality in animals (Boomsma 2007, 2009; Hughes et al. 2008).

In all cases, relatedness is determined by how groups are formed. A higher relatedness arises from: (a) offspring staying with their parents (termed subsociality) rather than individuals of the same generation aggregating together (termed parasociality or semisociality); and (b) either asexuality or lower levels of promiscuity. Taken together, these results support a fundamental role of how groups form, via its influence on relatedness, for the evolution of cooperative breeding, eusociality and now multicellularity (Boomsma 2013, 2007, 2009; Cornwallis et al. 2010; Lukas \& Clutton-Brock 2012; Hughes et al. 2008) (Figs. 2-4). Experimental evolution studies suggest that the form of group formation is similarly important for a range of cooperative behaviours in microorganisms (Griffin et al. 2004; MacLean \& Gudelj 2006; Diggle et al. 2007).

Furthermore, our data also support Boomsma's (Boomsma 2013, 2007, 2009) hypothesis that whilst a higher relatedness favours greater levels of cooperation, there is something special about clonality in asexual species or strict-lifetime monogamy in sexual species. At these extreme points, the offspring that can potentially stay and help their parents are equally related to the offspring they can help raise $\left(r_{h e l p}\right)$ and the offspring they could 
produce if they breed independently $\left(r_{\text {off }}\right)$, such that $r_{\text {help }}=r_{\text {off }}$ (Fig. 5). Consequently, as long as there is some ecological benefit to cooperation, this removes any conflict over whether to help or breed (Gardner \& Grafen 2009), and so facilitates the evolution of complete reproductive dependency between group members. The major evolutionary transitions to obligate multicellularity and obligate eusocial societies have only occurred when $r_{\text {help }}=r_{\text {off }}$ holds, due to group formation by offspring staying with parents, combined with either strict lifetime monogamy or asexual reproduction (Boomsma 2013, 2007, 2009; Grosberg \& Strathmann 2007; Hughes et al. 2008) (Fig. 2). The distinction between facultative and obligate multicellularity is of equal importance, and directly analogous to that between facultative and obligate eusociality (Boomsma 2013, 2009; Crespi \& Yanega 1995).

In contrast, when there is not strict-lifetime monogamy in sexual species, or groups form by individuals of the same generation aggregating together, then this leads to $r_{\text {help }}<r_{\text {off, }}$, and only transitions to facultative multicellularity or cooperative breeding have occurred (Boomsma 2013, 2007, 2009; Grosberg \& Strathmann 2007; Cornwallis et al. 2010; Lukas \& Clutton-Brock 2012) (Fig. 2). For example, the multi-clone fruiting bodies of Dictyostelid slime moulds, or the slightly promiscuous cooperative breeding vertebrates. This suggests that $r_{h e l p}=r_{\text {off }}$ can be a necessary precursor for 'fraternal' major transitions that involve cooperation between members of the same species. If $r_{\text {help }}<r_{\text {off }}$, then at some point in their life, potential helpers may do better by breeding independently, and so they are selected to retain the flexibility to do so (Boomsma 2013, 2007, 2009). Major transitions require, by definition, a complete reproductive dependency between group members (Maynard Smith \& Szathmáry 1995; Bourke 2011), and so the retention of this flexibility can stall a potential major transition (Fig. 5). 
a)

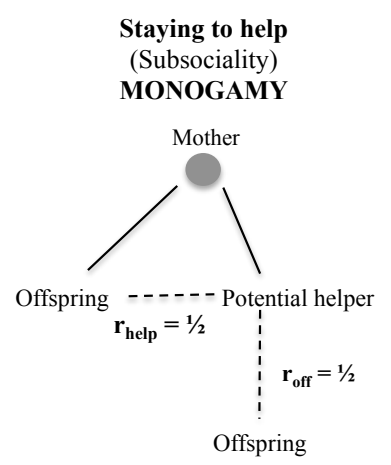

\section{Sexual Animals}
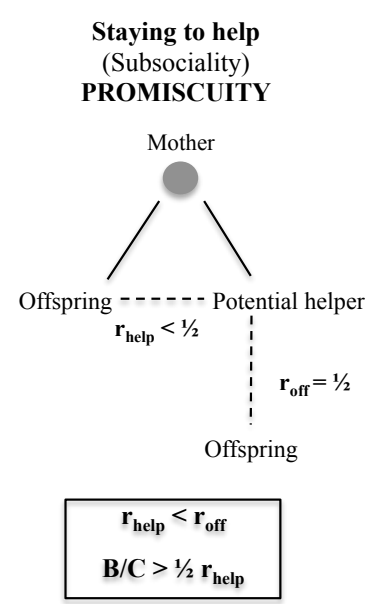
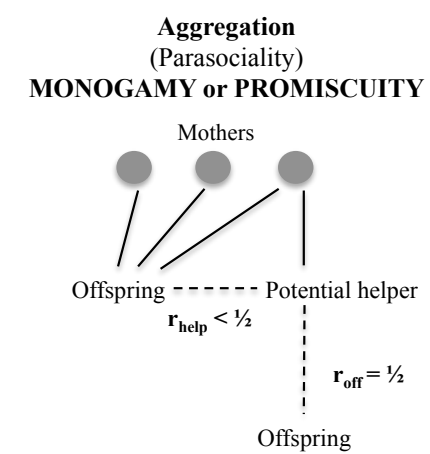

$\mathbf{r}_{\text {help }}=\mathbf{r}_{\text {off }}$
$\mathrm{B} / \mathrm{C}>\mathbf{1}$

$$
\begin{gathered}
\mathbf{r}_{\text {help }}<\mathbf{r}_{\text {off }} \\
\mathbf{B} / \mathbf{C}>1 / 2 \mathbf{r}_{\text {help }} \\
\hline
\end{gathered}
$$

b)

\section{Asexual Animals \& Cells}
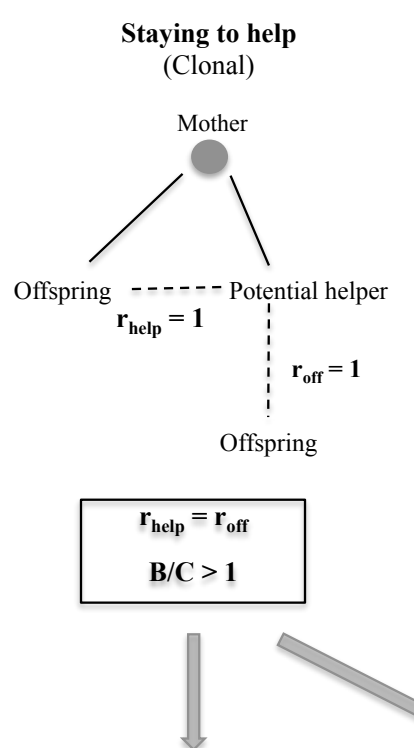

Obligate multicellularity and

Eusociality
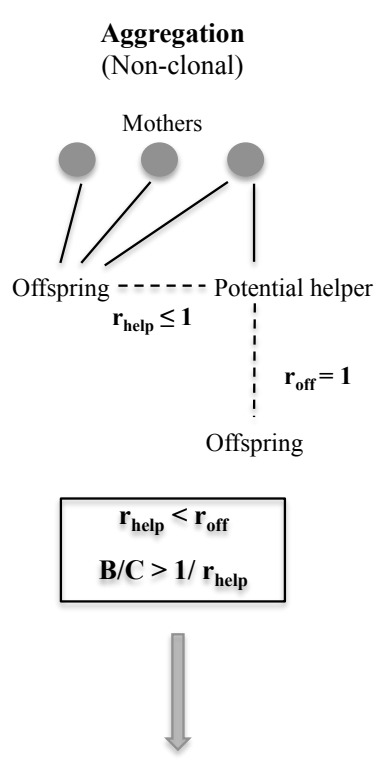

Facultative multicellularity and

Cooperative breeding 
Figure 5. Relatedness, group formation and evolutionary transitions. (a) In sexual animals, with strict lifetime monogamy, when groups form by offspring staying to help their mother (subsociality), then potential helpers are equally related to their own offspring $\left(r_{\text {off }}=1 / 2\right)$ and to their mother's offspring $\left(r_{h e l p}=1 / 2\right)$, who they could help raise. This means that there is no conflict over which to raise $\left(r_{\text {off }}=r_{\text {help }}\right)$, which facilitates the evolution of reproductive dependency between group members, and means that any small efficiency benefit of helping will favour altruistic cooperation (Boomsma 2007; Gardner \& Grafen 2009) $(B / C>1$; where $B$ and $C$ are the benefit to the and cost terms in Hamilton's (Hamilton 1964) rule). In contrast, if groups form by individuals from the same generation aggregating together (parasociality), such as sisters, or if females mate multiply during their lifetime (promiscuity), then potential helpers are more related to their own offspring $\left(r_{\text {off }}=1 / 2\right)$ than to the offspring who they could help raise $\left(r_{\text {help }}<1 / 2\right)$. In this case, it is much harder to evolve complete reproductive dependency between group members, and a larger efficiency benefit of helping is required to favour cooperation. (b) The same general predictions occur when considering group formation by cells, or asexual animals, such as aphids. Specifically: when groups form by offspring remaining with their parents, this leads to potential helpers being equally related to their own offspring and to the offspring that they could help raise $\left(r_{h e l p}=r_{o f f}\right)$, as they are all clonal $(r=1)$; when groups form by individuals aggregating together, this leads to potential helpers being more related to their own offspring than to the offspring who they could help raise $\left(r_{\text {help }}<r_{\text {off }}\right)$.

We thank: Bettina Schirrmeister for comments on cyanobacteria; Koos Boomsma, Andy Gardner, Ashleigh Griffin, Rick Grosberg, Elli Leadbeater, Andy Young and an anonymous referee for comments on the manuscript; the ERC, NERC and Vetenskapsrådet for funding. The dataset for this study can be found online. 


\section{Supplementary Information}

\section{Data Collection}

We searched the literature for information on the evolution and development of multicellularity in as many different taxa as possible. We searched for papers by: (1) searching for combinations of the following key words in the literature with Papers 2 (covering Scopus, Web of Knowledge, JSTOR, PubMed and Google Scholar): multicellular, multicellularity, colony formation, evolution, development, aggregation, subsocial, semisocial, clonal; (2) searching reviews of multicellularity; (3) doing forward and back searches on papers. Bettina Schirrmeister provided information on the life cycles of cyanobacteria.

We found data for 17 out of the 25 independent evolutionary transitions to multicellularity. Several groups (e.g. plants) include both complex and simple multicellular species. When analysing the evolutionary transitions (Fig. 4) we are interested in the highest level of complexity obtained, and so we classified each group according to the more complex species in the group. Whilst a single cell (unitary) stage leads to clonality (Grosberg \& Strathmann 1998, 2007), clonality can also occur in species with multicellular propagules, when the propagules are formed by cells remaining with their parents, as in some Cyanobacteria (Schirrmeister et al. 2011). This matters because, from an evolutionary theory perspective, the key distinction is whether groups are clonal $(r=1)$ or not $(r<1)$, and not just whether there is a single cell (unitary) stage (although they will be highly correlated (Grosberg \& Strathmann 1998, 2007)). 


\section{Statistical methods}

We examined whether the evolution of multicellularity was influenced by relatedness by testing if there were differences between clonal and non-clonal taxa for six different social traits: (1) obligate versus facultative multicellularity (binary distribution); (2) total number of cells (Gaussian after log transformation); (4) the number of cell types after controlling for total number of cells (Poisson distribution); (5) presence of sterile cells (binary distribution); (4) $\%$ of cells that are sterile in taxa with sterile soma (binomial distribution); (6) complex versus simple multicellularity (binary distribution). Our analyses involved four steps:

\section{Differences between clonal and non-clonal taxa in multicellular traits}

We analyzed if clonal and non-clonal taxa differed in traits 1 to 6 using Bayesian generalised linear models (BGLM) with Markov chain Monte Carlo (MCMC) estimation in MCMCglmm, R version 2.15.1 (Hadfield \& Nakagawa 2010). The results are presented in Table S1. In all models relatedness (clonal versus non-clonal) was entered as a fixed effect. For the analysis of cell types, the total number of cells (log and then Z-transformed) was included as a covariate. For some taxa information on the total number of cells was not available. To avoid excluding these data points from the analysis of cell types missing data was imputed as the mean value ( $=0$ after $\mathrm{Z}$ transformation) (Nakagawa $\&$ Freckleton 2010).

The parameter estimates we report are the posterior mode and $95 \%$ credible intervals (lower CI - upper CI), which were back-transformed to the scale of the response variable and marginalized over the residual variance. In all models the global intercept was removed to gain absolute parameter estimates for each level of the fixed effects. Estimates 
of the differences between the levels of fixed effects were calculated from a posterior distribution created by subtracting the estimates for each level obtained during each MCMC iteration (labelled difference in Tables S1-S2). Parameter estimates were considered statistically significant when $95 \%$ credible intervals did not include 0 and pMCMC values calculated in MCMCglmm (number of simulated cases that are $>0$ or $<0$ corrected for finite number of MCMC samples) were less than 0.05 .

1.2 Model specifications: We ran each analysis for 6000000 iterations with a burn-in of 1000000 and thinning interval of 1000 . These iteration settings were chosen as they minimized autocorrelation between posterior samples during test runs where we varied the number of iterations from 1 to 6 million, the burn-in from 0.5 to 5 million and the thinning interval from 100 to 1000 . For binary and binomial traits we used models with logit link functions and specified a fixed effect prior of $\mathrm{N}(0, \sigma 2$ units, $+\pi 2 / 3)$, which is relatively uniform on the logit scale. For binary response variables we fixed the residual variance to 1. For Poisson response variables we used log link functions and for Poisson and Gaussian traits we specified a prior of $\mathrm{V}=1, \mathrm{nu}=0.002$ for the residual variance.

1.3 Model checking: We checked the convergence of models by visually inspecting trace plots of MCMC chains and using Gelman-Rubin tests in the R package 'coda' (Gelman \& Rubin 1992; Plummer et al. 2006). For the Gelman-Rubin test we ran each analysis 3 times and used the Gelman-Rubin statistic (potential scale reduction factor; PSR) to compare within- and between-chain variance. When models have converged the PSR $<1.1$ and in all our analyses PSR was $<1.05$. Furthermore, for models using logit link functions we checked that the absolute value of the latent variable did not exceed 20 (Nakagawa \& Freckleton 2010). 


\section{Phylogenetic analyses of differences between clonal and non-clonal taxa in multicellular traits}

We obtained the data on multicellular traits from a very diverse range of taxa. We therefore repeated the analyses outlined in section 1, but included information on the nonindependence of data caused by the phylogenetic relationships between taxa (Nakagawa \& Freckleton 2010). We used Bayesian phylogenetic mixed models (BPMM) with MCMC estimation implemented in MCMCglmm. A phylogenetic tree of the taxa in our dataset (created from published sources - see 2.1) was entered as a random effect, which specifies a covariance matrix describing the relationships between taxa (Nakagawa \& Freckleton 2010). The output from these models is reported in exactly the same way as section 1 apart from parameter estimates were marginalized over the sum of the random effects (phylogenetic and residual variance) as opposed to only residual variance. The results are presented in Table S2.

2.1 Tree creation: We used phylogenies from the following sources to resolve polytomies in our dataset and create taxonomic structuring for the analyses: (1) between the three domains showing bacteria as the root, (Williams et al. 2012); (2) between the major groups of bacteria (Wu et al. 2009); (3) between major groups of eukaryotes (Burki \& Pawlowski 2006; Paps et al. 2012); (4) relationships within the fungi (James et al. 2006); (5) relationships within the red algae (Freshwater \& Rueness 1994); (6) relationships within the brown algae (Silberfeld et al. 2010); (7) relationships within the Volvocine algae (Hallmann 2011). We used these phylogenies because they were recent, included taxa in our dataset and were highly cited. 
We converted the information on taxonomy and topology into a phylogenetic tree using the R package 'ape' (Paradis et al. 2004). In some parts of the tree there were polytomies and we resolved these using the multi2d function in ape. In the areas of the tree with polytomies there was no variation in relatedness (all taxa involved in polytomies were either clonal or non-clonal) and there was no variation in obligate versus facultative reproduction, the presence of sterile soma or complex versus simple classifications of multicellularity. Therefore resolving these polytomies randomly had little influence on our results. We set all branch lengths equal to 1 (see section 4 for tests of robustness of our results to different branch length settings).

2.2 Model specifications: Model settings were the same as in section 1.2 apart from the prior specification for the extra random effect of phylogeny. We ran models using two different priors for random effects. First, we used an inverse gamma prior that is commonly used for random effects $(\mathrm{V}=1, \mathrm{nu}=0.002)$. Second, we ran models with parameter expanded priors (half-Cauchy priors following: $\mathrm{V}=1, \mathrm{nu}=1$, alpha.mu $=0$, alpha. $\left.\mathrm{V}=25^{\wedge} 2\right)$ as sometimes variance components were close to 0 (Gelman \& Rubin 1992).The inverse gamma prior led to better convergence as measured by autocorrelation between posterior samples and therefore we only present results from these models.

2.3 Model checking: We checked models in exactly the same way as in section 1.3. We also examined the convergence of estimates of random effects by visually inspecting trace plots of the MCMC chains and by examining autocorrelation between posterior samples.

3. Robustness of results to assumptions of causality and allowing relatedness to evolve across the phylogenetic tree 
The analyses presented in sections $1 \& 2$ assume that relatedness does not evolve and causally effects each of the social traits (response variables). Theoretically, the causal effect of relatedness on the evolution of social traits is strongly supported, but variation in relatedness amongst cells may change over evolutionary time. We therefore modeled the phylogenetic correlation between relatedness and each of the six social traits allowing relatedness and the social traits to evolve across the tree, whilst also relaxing the assumption of causality. The results are presented in Table S3.

We analyzed phylogenetic correlations using the threshold model described by Felsenstein (Felsenstein 2012). The threshold model allows the correlated evolution of discrete characters, such as clonal versus non-clonal and obligate versus facultative reproduction, to be modeled by assuming there is some unobserved quantitative character (liability) that underlies discrete characters: at a certain threshold in liability the phenotype switches from one state to another. This is well suited to modeling evolutionary changes in discrete characters because it allows the underlying probability of displaying a character to evolve rather than assuming changes between states at each node occur with equal probability across tree, as with continuous-time Markov models. For example, the probability of evolving obligate multicellular reproduction is more likely if taxa are within clades dominated obligate reproduction in comparison to the average across the tree (Felsenstein 2012; Hadfield 2010). Furthermore, it also allows the phylogenetic correlation between discrete and continuous traits, such as clonal versus non-clonal and total number of cells, to be estimated. We used a Bayesian implementation of the threshold model, threshBayes, in the R package 'phytools' (Revell 2011) to analyze our data. Prior to analysis the number of cells and the number of cell types were log and then Z-transformed and the proportion of sterile cells was square root arcsine transformed. 
3.1 Model specifications: We ran each analysis for 6000000 iterations with a burn-in of 1000000 and thinning interval of 1000 . We used the default prior settings that specify exponential priors for the evolutionary variances associated with each trait, normal priors for the ancestral state of each trait and a uniform prior for the correlation between the two traits.

3.2 Model checking: We checked models with the same diagnostics as section 2.3.

\section{Robustness of results to assumptions of tree branch lengths}

Phylogenetic analyses using trees with equal branch lengths can over estimate the strength of association between variables (Purvis et al. 1994). We therefore examined the robustness of our results to assumptions of branch lengths in two ways.

4.1 Repeating analyses using different tree transformations: We repeated the analyses outlined in sections $2 \& 3$ twice, once where the branches of the phylogenetic tree had been transformed to simulate an early burst of diversification (Fig. S1a. Referred to as 'early burst' in tables) and once to simulate a late burst of diversification (Fig. S1c. Referred to as 'late burst' in tables). We created the early and late burst trees using Pagel's $\delta$ transformations (Early burst $\delta=0.2$, Late burst $\delta=1.5$ ) implemented through the deltaTree function in the R package 'Geiger' (Harmon et al. 2008; Pagel 1994; Pagel 1999). The results of the MCMCglmm analyses are presented in Table S4 and those of threshBayes are given in Table S5. 
4.2 Removing branch lengths: ancestral reconstruction using maximum parsimony: We used maximum parsimony, which does not require a tree with branch lengths, to reconstruct ancestral states of relatedness using the MPR function in the R package 'ape'. After reconstructing ancestral states we identified independent evolutionary transitions between clonal and non-clonal multicellularity. We tested whether clonal and non-clonal taxa differed in their social traits using Wilcoxon paired rank sum tests of the mean phenotype of the clonal taxa and the mean phenotype of the non-clonal taxa involved in each independent comparison. The results of these analyses are presented in Table S6. 


\section{Supplementary Figures}

Figure S1: Ancestral reconstruction of the relationships between clonal (black tip labels and edges) and non-clonal (red tip labels and edges) taxa. Where data were missing for the tips (e.g for the total number of cells and cell types) no symbols were plotted. Grey dots and lines represent ambiguous reconstructions where nodes could not be assigned to one state or the other (posterior probability between 0.1 and 0.9 : state 0 was classified as $<0.1$ posterior probability and state 1 as $>0.9$ posterior probability). pMCMC values are taken from Table S2 and represent differences between clonal and nonclonal taxa in each of the social traits.

(a) Obligate vs. Facultative reproduction

$$
(\mathrm{pMCMC}=0.0002)
$$

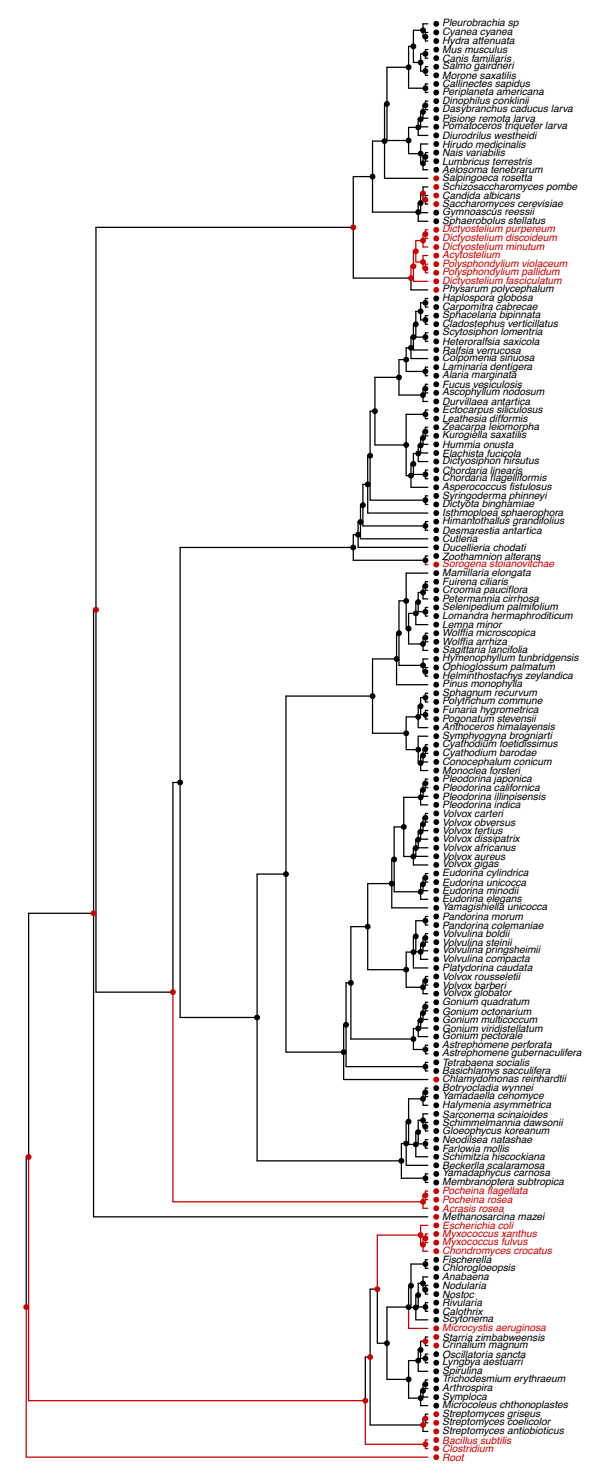

Figure S1a: Ancestral reconstruction of the relationships between clonal (black tip labels and edges) and non-clonal (red tip labels and edges) taxa and obligate versus facultative multicellularity (obligate $=$ black dots at tips and nodes, facultative $=$ red dots at tips and nodes) 
(b) Number of cells

$(\mathrm{pMCMC}=0.27)$

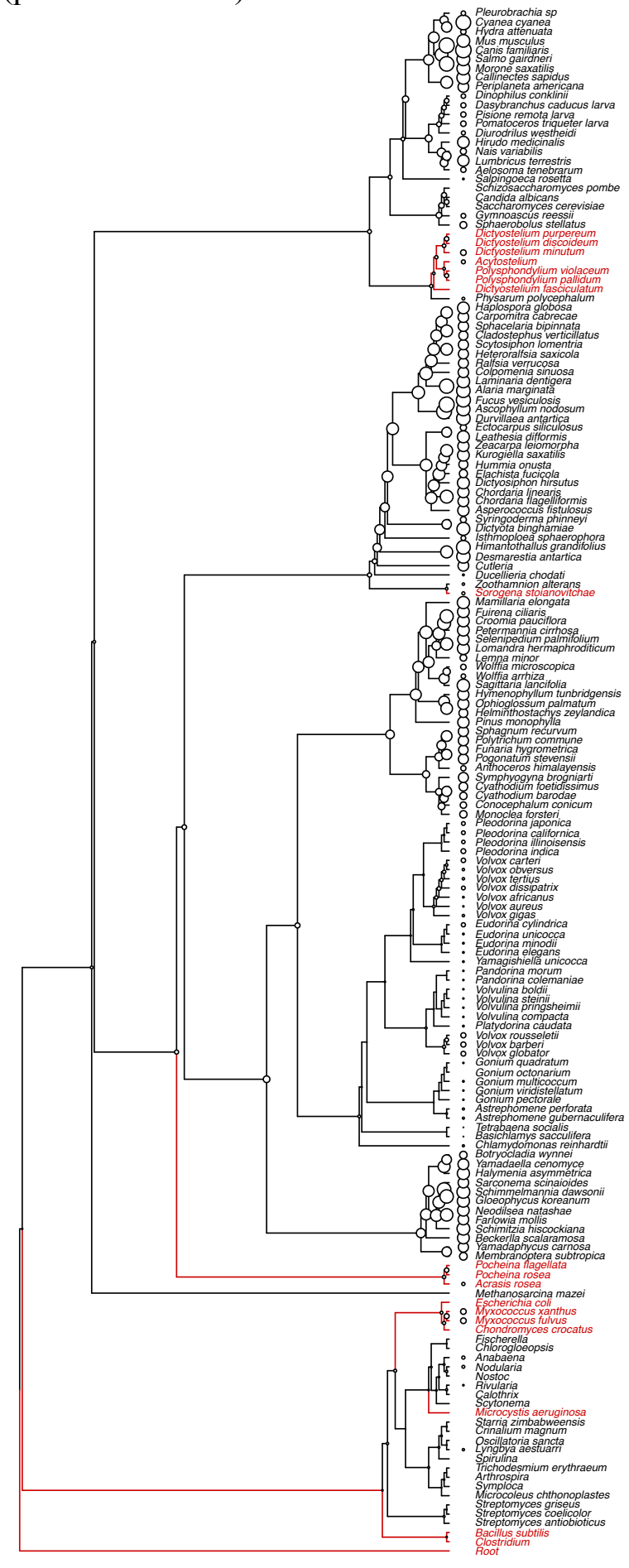

Figure S1b: Ancestral reconstruction of the relationships between clonal (black tip labels and edges) and non-clonal (red tip labels and edges) taxa and total number of cells (size of dots at tips and nodes $=\log$ number of cells), 
(c) Number of cell types

$(\mathrm{pMCMC}=0.0008)$

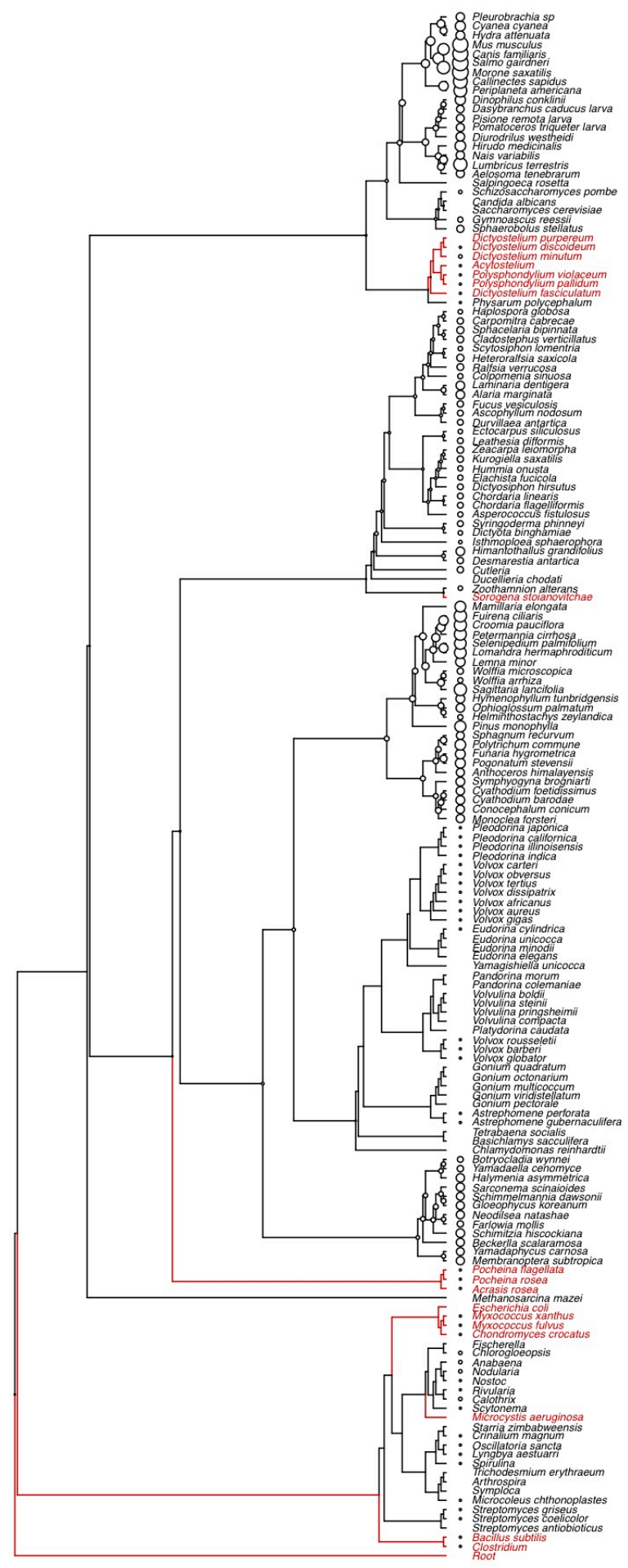

Figure S1c: Ancestral reconstruction of the relationships between clonal (black tip labels and edges) and non-clonal (red tip labels and edges) taxa and number of cell types (size of dots at tips and nodes $=\log$ number of cell types). 
(d) Probability of sterile soma

(pMCMC $=0.02$ )

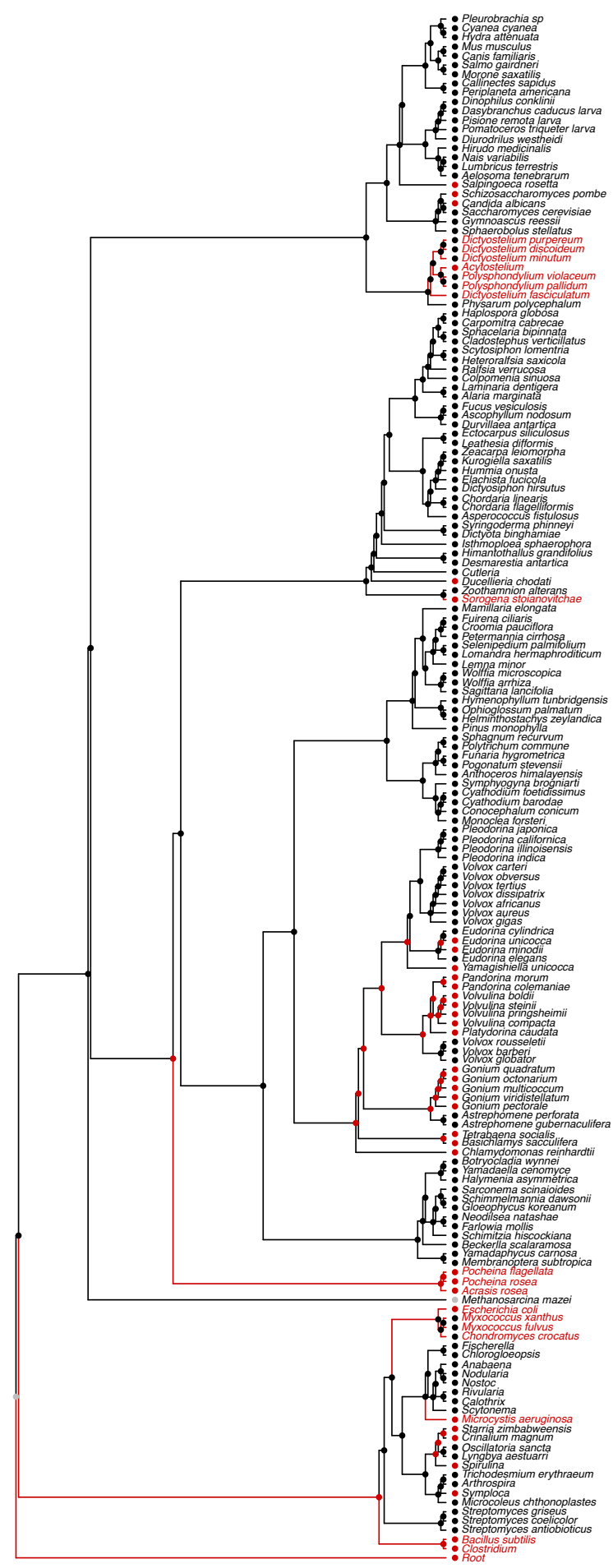

Figure S1d: Ancestral reconstruction of the relationships between clonal (black tip labels and edges) and non-clonal (red tip labels and edges) taxa and probability of sterile soma $($ sterile $=$ black dots at tips and nodes, non-sterile $=$ red dots at tips and nodes). 
(e) \% cells that are sterile

$(\mathrm{pMCMC}=0.26)$

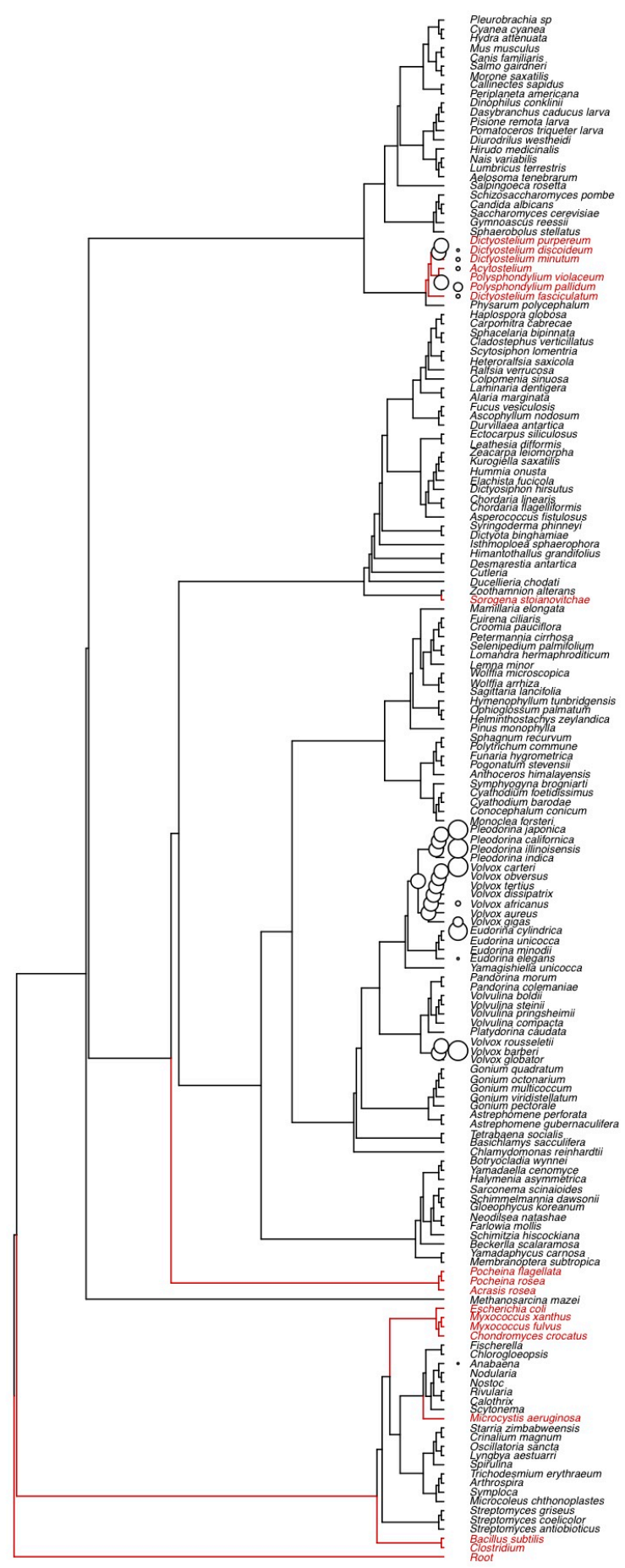

Figure S1e: Ancestral reconstruction of the relationships between clonal (black tip labels and edges) and non-clonal (red tip labels and edges) taxa and \% cells that are sterile (size of dots at tips and nodes $=\%$ ). 
(f) Complex versus simple multicellularity $(\mathrm{pMCMC}=0.21)$

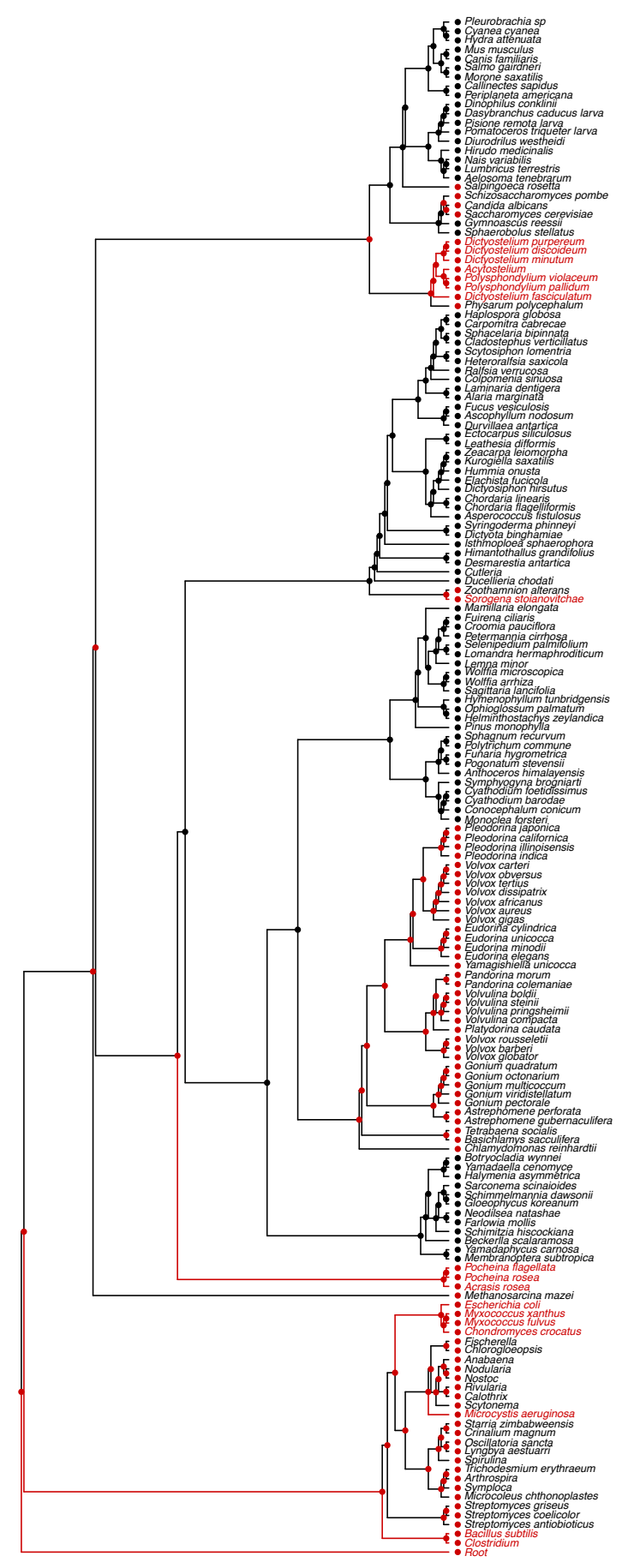

Figure S1f: Ancestral reconstruction of the relationships between clonal (black tip labels and edges) and non-clonal (red tip labels and edges) taxa and complex versus simple multicellularity $($ complex $=$ black dots at tips and nodes, simple $=$ red dots at tips and nodes). 
Figure S2: The (a) early burst ( $\delta=0.2)$, (b) equal length and (c) late $(\delta=1.5)$ burst trees used for analyses examining the robustness of results to assumptions about branch lengths (related to Figure 2 in main text).

(a)

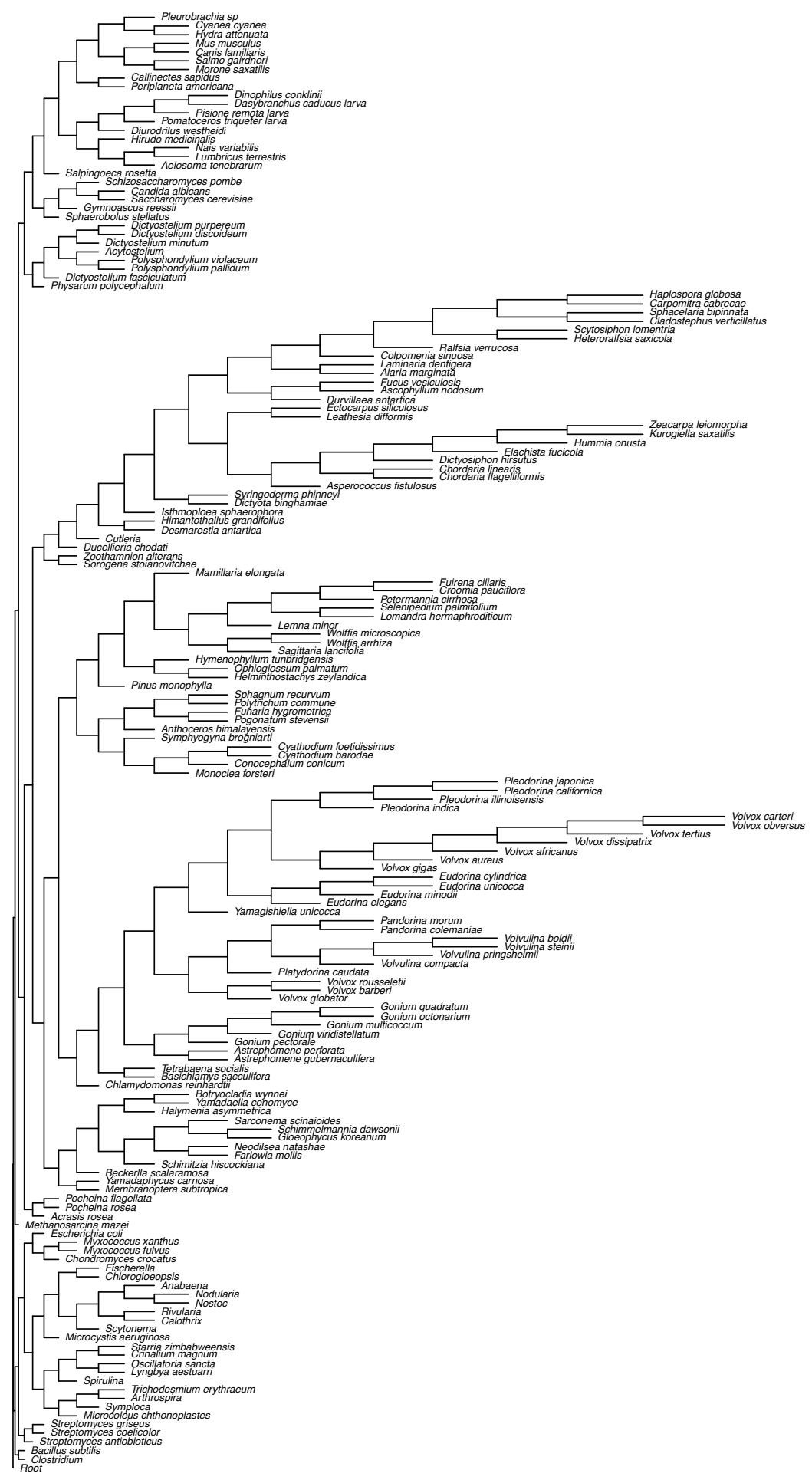


(b)

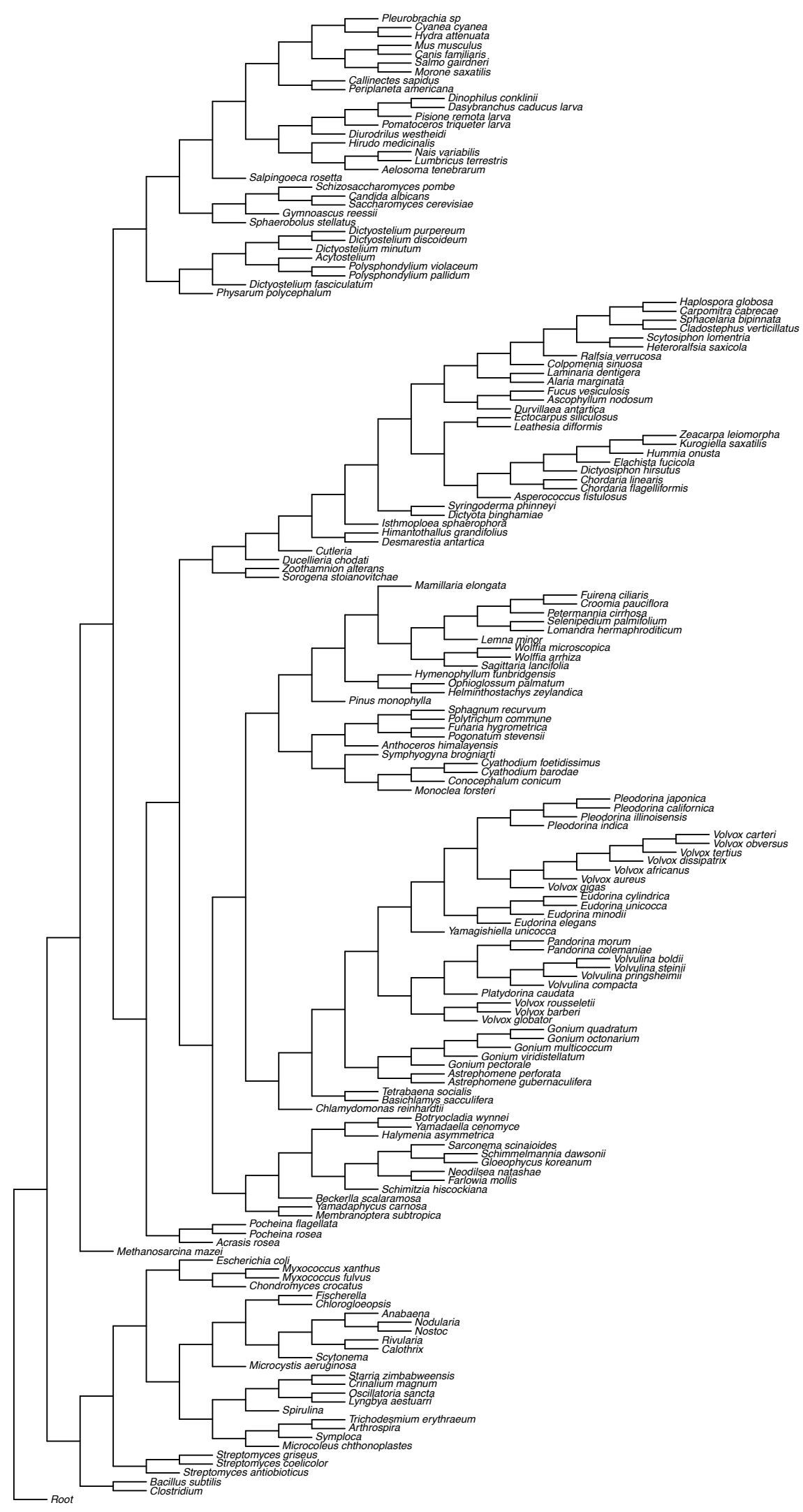


(c)

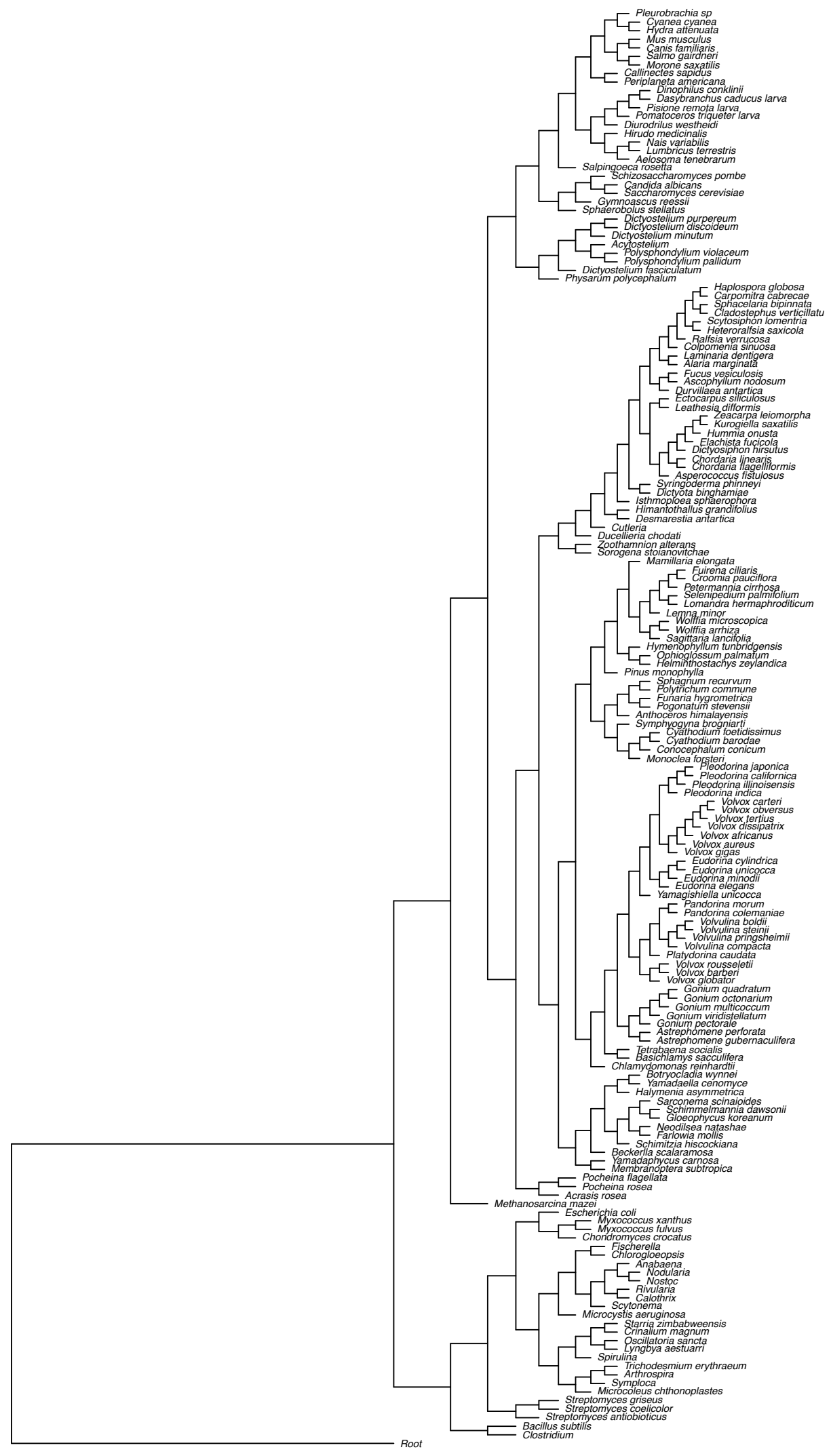


a)

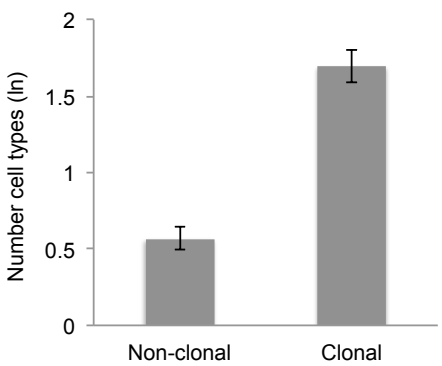

c)

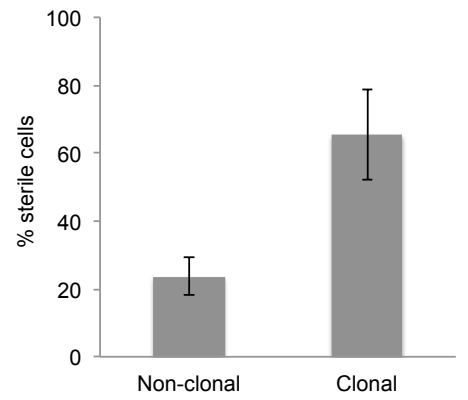

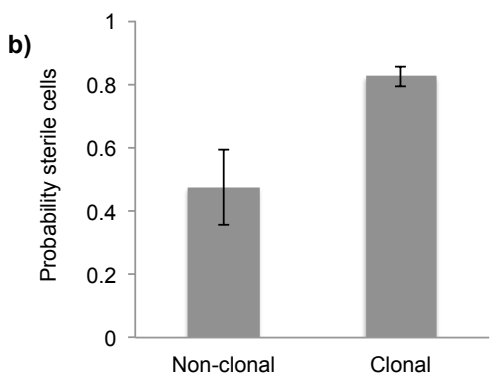

d)

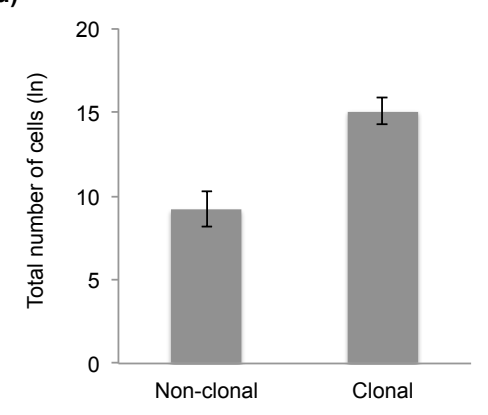

e)
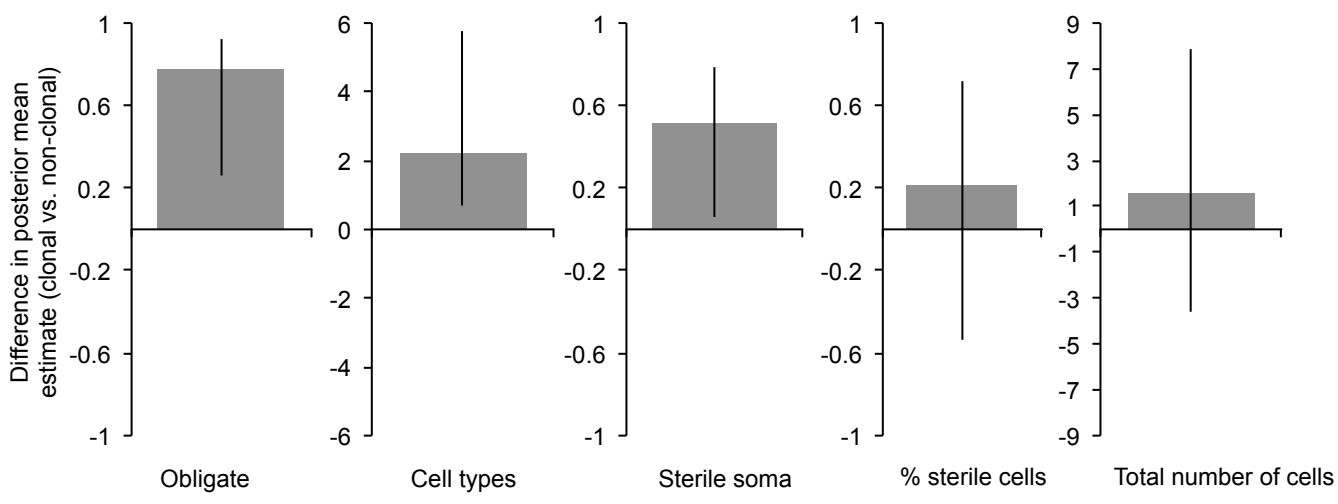

Figure S3. Relatedness and sociality in multicellular groups. The graphs show, for multicellular groups that are either clonal or potentially non-clonal, the: (a) number of cell types; (b) probability of having sterile cells; (c) percentage of sterile cells in species with a sterile cells; (d) total number of cells in the group. The graphs show the raw data, with means and standard errors calculated assuming species as independent data points. (e) shows the difference between clonal and non-clonal (posterior mean estimate from $\mathrm{BPMM} \pm 95 \%$ credible interval) species for five measures of sociality: the probability of being obligate, number of cell types, probability of having a sterile soma, $\%$ sterile cells in species with a sterile soma and total number of cells (related to Figure 3 in the main text). 


\section{List of Supplementary Tables}

Table S1: Analyses of the effect of relatedness (clonal versus non-clonal) on social traits in multicellular groups not accounting for phylogenetic relationships between species conducted using MCMCglmm.

Table S2: Analyses of the effect of relatedness (clonal versus non-clonal) on social traits in multicellular groups taking into account phylogenetic relationships between species conducted using MCMCglmm.

Table S3: Estimates of the phylogenetic correlation between relatedness (clonal versus non-clonal) and different social traits in multicellular group analysed using threshBayes.

Table S4: The influence of assumptions about branch lengths on the estimated effect of relatedness (clonal versus non-clonal) on social traits in multicellular groups analysed using MCMCglmm.

Table S5: The influence of assumptions about branch lengths on the estimated phylogenetic correlation between relatedness (clonal versus non-clonal) and social traits in multicellular groups analysed using threshBayes.

Table S6: Wilcoxon paired rank sum tests of differences in social traits across independent evolutionary transitions between clonal and non-clonal states identified using maximum parsimony ancestral reconstruction. 
Table S1: Analyses of the effect of relatedness (clonal versus non-clonal) on social traits in multicellular groups not accounting for phylogenetic relationships between species conducted using MCMCglmm.

\begin{tabular}{|c|c|c|c|c|c|c|}
\hline Trait & Species & Effect & Posterior mode & $\begin{array}{c}\text { Lower } \\
\text { CI }\end{array}$ & $\begin{array}{c}\text { Upper } \\
\text { CI }\end{array}$ & $P$ diff \\
\hline \multirow{3}{*}{$\begin{array}{l}\text { 1. Obligate versus } \\
\text { facultative }\end{array}$} & 168 & Clonal & 0.91 & 0.87 & 0.95 & \\
\hline & & Non-clonal & 0.03 & 0.0006 & 0.13 & \\
\hline & & Difference & 0.89 & 0.77 & 0.94 & $<0.0001$ \\
\hline \multirow[t]{3}{*}{ 2. Number of cells } & 133 & Clonal & 15.16 & 13.67 & 16.73 & \\
\hline & & Non-clonal & 8.36 & 1.33 & 14.39 & \\
\hline & & Difference & 6.73 & 0.55 & 13.99 & 0.02 \\
\hline \multirow[t]{4}{*}{ 3. Number of cell types } & 162 & Clonal & 7.23 & 6.36 & 8.83 & \\
\hline & & Non-clonal & 2.64 & 1.42 & 4.12 & \\
\hline & & Difference & 4.91 & 2.95 & 6.65 & $<0.0001$ \\
\hline & & $\begin{array}{l}\text { Number of } \\
\text { cells }\end{array}$ & 3.50 & 2.88 & 4.24 & $<0.0001$ \\
\hline \multirow[t]{3}{*}{$\begin{array}{l}\text { 4. Probability of sterile } \\
\text { soma }\end{array}$} & 167 & Clonal & 0.83 & 0.76 & 0.88 & \\
\hline & & Non-clonal & 0.45 & 0.26 & 0.69 & \\
\hline & & Difference & 0.30 & 0.14 & 0.58 & 0.001 \\
\hline \multirow[t]{3}{*}{ 5. $\%$ cells that are sterile } & 48 & Clonal & 0.74 & 0.49 & 0.86 & \\
\hline & & Non-clonal & 0.35 & 0.17 & 0.65 & \\
\hline & & Difference & 0.35 & -0.02 & 0.61 & 0.05 \\
\hline \multirow[t]{3}{*}{$\begin{array}{l}\text { 6. Complex versus } \\
\text { simple }\end{array}$} & 168 & Clonal & 0.58 & 0.50 & 0.66 & \\
\hline & & Non-clonal & 0.03 & 0.00 & 0.14 & \\
\hline & & Difference & 0.55 & 0.41 & 0.65 & $<0.0001$ \\
\hline
\end{tabular}

Table S2: Analyses of the effect of relatedness (clonal versus non-clonal) on social traits in multicellular groups taking into account phylogenetic relationships between species conducted using MCMCglmm.

\begin{tabular}{|c|c|c|c|c|c|}
\hline \multirow[t]{2}{*}{ Trait } & \multirow[t]{2}{*}{ Effect } & \multirow[t]{2}{*}{ Posterior mode } & \multirow{2}{*}{$\begin{array}{c}\text { Lower } \\
\text { CI }\end{array}$} & \multirow{2}{*}{$\begin{array}{c}\text { Upper } \\
\text { CI }\end{array}$} & \multirow[t]{2}{*}{$P$ diff } \\
\hline & & & & & \\
\hline \multirow{3}{*}{$\begin{array}{l}\text { 1. Obligate versus } \\
\text { facultative }\end{array}$} & Clonal & 0.80 & 0.43 & 0.97 & \\
\hline & Non-clonal & 0.02 & 0.002 & 0.35 & \\
\hline & Difference & 0.78 & 0.26 & 0.92 & 0.0002 \\
\hline
\end{tabular}




\begin{tabular}{|c|c|c|c|c|c|}
\hline & Phylogeny $^{1}$ & 0.36 & 0.08 & 0.87 & \\
\hline \multirow[t]{4}{*}{ 2. Number of cells } & Clonal & 4.98 & -2.94 & 12.04 & \\
\hline & Non-clonal & 2.55 & -3.05 & 8.96 & \\
\hline & Difference & 1.55 & -3.60 & 7.84 & 0.27 \\
\hline & Phylogeny $^{2}$ & 0.99 & 0.34 & 0.99 & \\
\hline \multirow[t]{5}{*}{ 3. Number of cell types } & Clonal & 4.33 & 2.12 & 8.53 & \\
\hline & Non-clonal & 1.71 & 0.80 & 3.69 & \\
\hline & Difference & 2.20 & 0.67 & 5.79 & 0.0008 \\
\hline & Number of cells & 1.98 & 1.68 & 2.23 & $<\mathbf{0 . 0 0 0 1}$ \\
\hline & Phylogeny $^{2}$ & 0.97 & 0.65 & 0.99 & \\
\hline \multirow[t]{4}{*}{$\begin{array}{l}\text { 4. Probability of sterile } \\
\text { soma }\end{array}$} & Clonal & 0.79 & 0.42 & 0.97 & \\
\hline & Non-clonal & 0.19 & 0.03 & 0.53 & \\
\hline & Difference & 0.51 & 0.06 & 0.79 & 0.02 \\
\hline & Phylogeny $^{1}$ & 0.66 & 0.29 & 0.95 & \\
\hline \multirow[t]{4}{*}{ 5. $\%$ cells that are sterile } & Clonal & 0.61 & 0.06 & 0.92 & \\
\hline & Non-clonal & 0.34 & 0.03 & 0.86 & \\
\hline & Difference & 0.21 & -0.54 & 0.72 & 0.39 \\
\hline & Phylogeny $^{2}$ & 0.99 & 0.06 & 0.99 & \\
\hline \multirow[t]{4}{*}{ 6. Complex versus simple ${ }^{3}$} & Clonal & 0.50 & 0.20 & 0.74 & \\
\hline & Non-clonal & 0.53 & 0.38 & 0.92 & \\
\hline & Difference & -0.10 & -0.57 & 0.17 & 0.21 \\
\hline & Phylogeny $^{1}$ & 0.97 & 0.56 & 0.99 & \\
\hline
\end{tabular}

\footnotetext{
${ }^{1}$ Intraclass correlation coefficient of phylogeny.

${ }^{2}$ Proportion of residual variation explained by phylogeny.

${ }^{3}$ Should be interpreted with caution, logit link function overloaded as latent variable exceeded 20.
} 
Table S3: Estimates of the phylogenetic correlation between relatedness (clonal versus non-clonal) and different social traits in multicellular group analysed using threshBayes.

\begin{tabular}{lcccc}
\hline Trait & $\begin{array}{c}\text { Phylogenetic correlation } \\
\text { with relatedness (posterior }\end{array}$ & Lower & Upper CI & P \\
& mode) & & & \\
& & & & \\
& 0.73 & 0.39 & 0.97 & 0.08 \\
\hline 1. Obligate versus facultative & 0.36 & -0.11 & 0.68 & $\mathbf{0 . 0 0 3}$ \\
2. Number of cells & 0.59 & 0.18 & 0.74 & $\mathbf{0 . 0 0 9}$ \\
$\begin{array}{l}\text { 3. Number of cell types } \\
\text { 4. Probability of sterile soma }\end{array}$ & 0.58 & 0.11 & 0.73 & 0.34 \\
5. \% cells that are sterile & 0.31 & -0.59 & 0.81 & 0.09 \\
6. Complex versus simple & 0.26 & -0.09 & 0.60 &
\end{tabular}


Table S4: The influence of assumptions about branch lengths on the estimated effect of relatedness (clonal versus non-clonal) on social traits in multicellular groups analysed using MCMCglmm.

\begin{tabular}{|c|c|c|c|c|c|c|c|c|c|}
\hline Trait & $\begin{array}{l}\text { Branch } \\
\text { lengths }\end{array}$ & DIC & $\begin{array}{l}\text { Posterior mode of } \\
\text { difference between } \\
\text { clonal and non- } \\
\text { clonal }\end{array}$ & $\begin{array}{c}\text { Lower } \\
\text { CI }\end{array}$ & $\begin{array}{c}\text { Upper } \\
\text { CI }\end{array}$ & $P$ diff & $\begin{array}{c}\text { Phylogenetic } \\
\text { posterior } \\
\text { mode }\end{array}$ & $\begin{array}{c}\text { Lower } \\
\text { CI }\end{array}$ & $\begin{array}{c}\text { Upper } \\
\text { CI }\end{array}$ \\
\hline \multirow[t]{3}{*}{$\begin{array}{l}\text { 1. Obligate } \\
\text { versus } \\
\text { facultative }\end{array}$} & $\begin{array}{l}\text { Equal } \\
\text { length }\end{array}$ & 46.7 & 0.78 & 0.26 & 0.92 & 0.0002 & 0.36 & 0.08 & 0.87 \\
\hline & $\begin{array}{l}\text { Early } \\
\text { burst }\end{array}$ & 53.8 & 0.54 & 0.20 & 0.81 & 0.001 & 0.77 & 0.23 & 0.96 \\
\hline & $\begin{array}{l}\text { Late } \\
\text { burst }\end{array}$ & 42.9 & 0.64 & 0.22 & 0.88 & 0.001 & 0.68 & 0.32 & 0.96 \\
\hline \multirow[t]{3}{*}{$\begin{array}{l}\text { 2. Number } \\
\text { of cells }\end{array}$} & $\begin{array}{l}\text { Equal } \\
\text { length }\end{array}$ & 196.8 & 1.55 & -3.60 & 7.84 & 0.27 & 0.99 & 0.34 & 0.99 \\
\hline & $\begin{array}{l}\text { Early } \\
\text { burst }\end{array}$ & 810.6 & 4.78 & -0.31 & 8.78 & 0.06 & 0.47 & 0.23 & 0.69 \\
\hline & $\begin{array}{l}\text { Late } \\
\text { burst }\end{array}$ & 81.5 & 2.25 & -6.82 & 7.75 & 0.47 & 0.99 & 0.80 & 0.99 \\
\hline \multirow{3}{*}{$\begin{array}{l}\text { 3. Number } \\
\text { of cell } \\
\text { types }\end{array}$} & $\begin{array}{l}\text { Equal } \\
\text { length }\end{array}$ & 730.4 & 2.20 & 0.67 & 5.79 & 0.0008 & 0.97 & 0.65 & 0.99 \\
\hline & $\begin{array}{l}\text { Early } \\
\text { burst }\end{array}$ & 743.8 & 2.99 & 1.48 & 4.97 & $<0.0001$ & 0.99 & 0.84 & 0.99 \\
\hline & $\begin{array}{l}\text { Late } \\
\text { burst }\end{array}$ & 728.1 & 1.66 & 0.07 & 16.51 & 0.003 & 0.99 & 0.89 & 0.99 \\
\hline \multirow{3}{*}{$\begin{array}{l}4 . \\
\text { Probability } \\
\text { of sterile } \\
\text { soma }\end{array}$} & $\begin{array}{l}\text { Equal } \\
\text { length }\end{array}$ & 69.2 & 0.51 & 0.06 & 0.79 & 0.02 & 0.66 & 0.29 & 0.95 \\
\hline & $\begin{array}{l}\text { Early } \\
\text { burst }\end{array}$ & 61.8 & 0.27 & 0.02 & 0.58 & 0.02 & 0.98 & 0.59 & 0.99 \\
\hline & $\begin{array}{l}\text { Late } \\
\text { burst }\end{array}$ & 63.3 & 0.23 & -0.04 & 0.54 & 0.05 & 0.98 & 0.68 & 0.99 \\
\hline \multirow[t]{3}{*}{$\begin{array}{l}5 . \% \text { cells } \\
\text { that are } \\
\text { sterile }\end{array}$} & $\begin{array}{l}\text { Equal } \\
\text { length }\end{array}$ & 1002.9 & 0.21 & -0.54 & 0.72 & 0.39 & 0.99 & 0.06 & 0.99 \\
\hline & $\begin{array}{l}\text { Early } \\
\text { burst }\end{array}$ & 1002.9 & 0.09 & -0.35 & 0.63 & 0.33 & 0.99 & 0.52 & 0.99 \\
\hline & $\begin{array}{l}\text { Late } \\
\text { burst }\end{array}$ & 1003.0 & 0.25 & -0.40 & 0.58 & 0.32 & 0.99 & 0.001 & 0.99 \\
\hline \multirow[t]{3}{*}{$\begin{array}{c}\text { 6. Complex } \\
\text { versus } \\
\text { simple } \\
1\end{array}$} & $\begin{array}{l}\text { Equal } \\
\text { length }\end{array}$ & 15.7 & -0.10 & -0.57 & 0.17 & 0.21 & 0.97 & 0.56 & 0.99 \\
\hline & $\begin{array}{l}\text { Early } \\
\text { burst }\end{array}$ & 23.4 & -0.05 & -0.35 & 0.09 & 0.15 & 0.99 & 0.76 & 0.99 \\
\hline & $\begin{array}{l}\text { Late } \\
\text { burst }\end{array}$ & 11.05 & -0.03 & -0.31 & 0.14 & 0.28 & 0.99 & 0.89 & 0.99 \\
\hline
\end{tabular}

\footnotetext{
${ }^{1}$ Should be interpreted with caution, logit link function overloaded as latent variable exceeded 20.
} 
Table S5: The influence of assumptions about branch lengths on the estimated phylogenetic correlation between relatedness (clonal versus non-clonal) and social traits in multicellular groups analysed using threshBayes.

\begin{tabular}{|c|c|c|c|c|c|c|}
\hline Trait & $\begin{array}{l}\text { Branch } \\
\text { lengths }\end{array}$ & $\log L$ & $\begin{array}{c}\text { Phylogenetic } \\
\text { correlation with } \\
\text { relatedness (posterior } \\
\text { mode) }\end{array}$ & $\begin{array}{c}\text { Lower } \\
\text { CI }\end{array}$ & $\begin{array}{c}\text { Upper } \\
\text { CI }\end{array}$ & $P$ diff \\
\hline \multirow[t]{3}{*}{$\begin{array}{l}\text { 1. Obligate versus } \\
\text { facultative }\end{array}$} & Equal length & -606.7 & 0.73 & 0.39 & 0.97 & $<0.0001$ \\
\hline & Early burst & -449.9 & 0.80 & 0.49 & 0.94 & $<0.0001$ \\
\hline & Late burst & -356.5 & 0.70 & 0.32 & 0.89 & 0.002 \\
\hline \multirow[t]{3}{*}{ 2. Number of cells } & Equal length & -674.4 & 0.36 & -0.11 & 0.68 & 0.08 \\
\hline & Early burst & -645.5 & 0.51 & 0.07 & 0.70 & 0.01 \\
\hline & Late burst & -558.8 & 0.23 & -0.25 & 0.69 & 0.18 \\
\hline \multirow[t]{3}{*}{ 3. Number of cell types } & Equal length & -381.9 & 0.59 & 0.18 & 0.74 & 0.003 \\
\hline & Early burst & -356.6 & 0.53 & 0.25 & 0.75 & 0.0004 \\
\hline & Late burst & -252.8 & 0.57 & 0.17 & 0.77 & 0.005 \\
\hline \multirow[t]{3}{*}{$\begin{array}{l}\text { 4. Probability of sterile } \\
\text { soma }\end{array}$} & Equal length & -617.7 & 0.58 & 0.11 & 0.73 & 0.009 \\
\hline & Early burst & -489.3 & 0.50 & 0.17 & 0.74 & 0.005 \\
\hline & Late burst & -328.1 & 0.44 & 0.09 & 0.77 & 0.016 \\
\hline \multirow[t]{3}{*}{$\begin{array}{l}5 . \% \text { cells that are } \\
\text { sterile }\end{array}$} & Equal length & -35.2 & 0.31 & -0.59 & 0.81 & 0.34 \\
\hline & Early burst & -29.4 & 0.50 & -0.46 & 0.82 & 0.28 \\
\hline & Late burst & -26.6 & 0.39 & -0.62 & 0.87 & 0.36 \\
\hline \multirow[t]{3}{*}{$\begin{array}{l}\text { 6. Complex versus } \\
\text { simple }\end{array}$} & Equal length & -625.2 & 0.26 & -0.09 & 0.60 & 0.09 \\
\hline & Early burst & -485.2 & 0.40 & 0.10 & 0.69 & 0.01 \\
\hline & Late burst & -334.0 & 0.45 & -0.009 & 0.78 & 0.05 \\
\hline
\end{tabular}


Table S6: Wilcoxon paired rank sum tests of differences in social traits across independent evolutionary transitions between clonal (black) and non-clonal states (red) identified using maximum parsimony ancestral reconstruction.

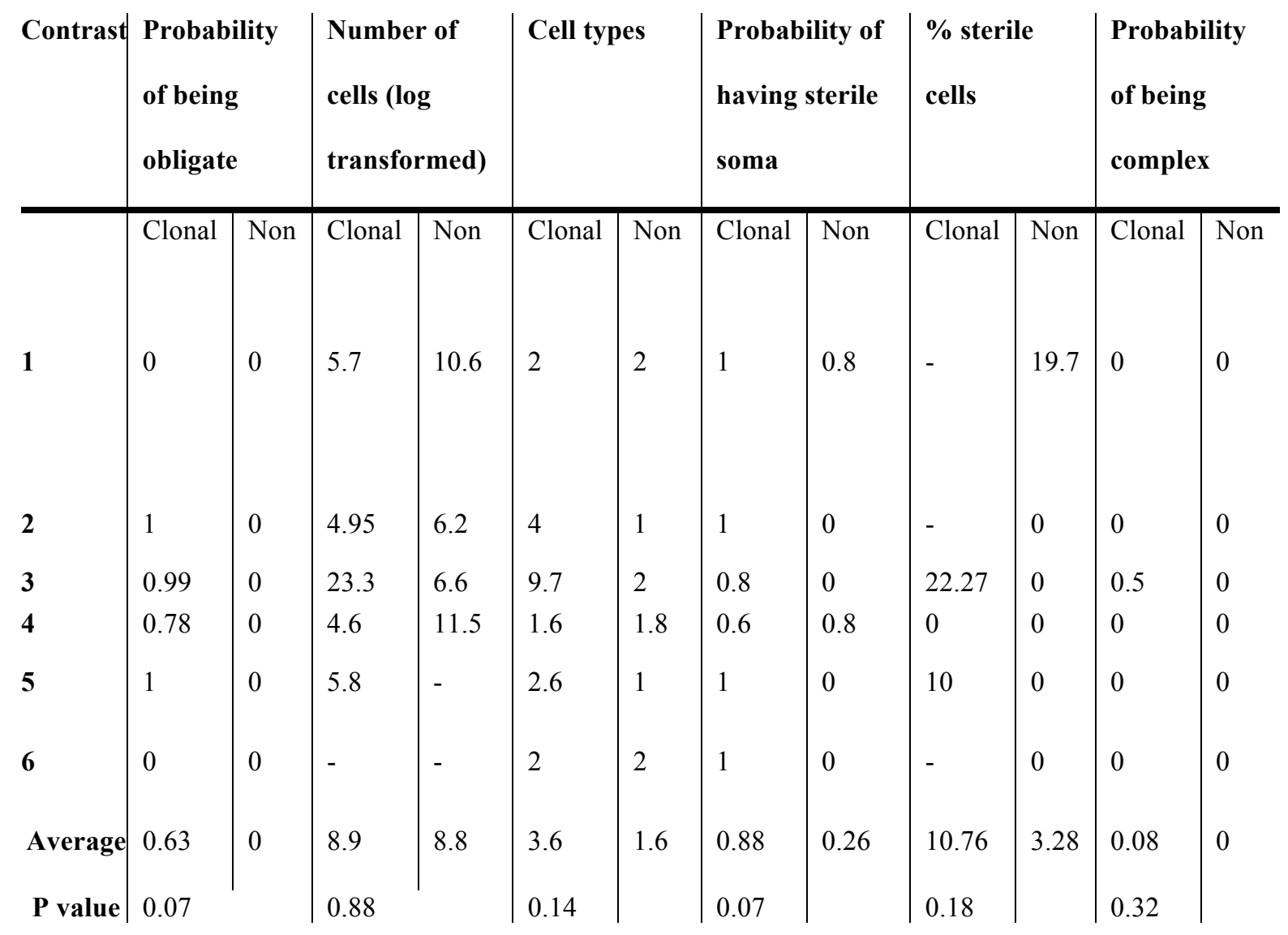




\title{
Chapter 3: Multicellular group formation in Chlorella vulgaris
}

\begin{abstract}
To understand the evolution of multicellular organisms, we need to examine why individual cells would cooperate and form a multicellular group. It has been suggested that predation pressure may be crucial in promoting multicellular group formation, where individual cells benefit by lowering their chance of being eaten, and there is support for this in unicellular algae and bacteria. We use a new experimental system using the green alga Chlorella vulgaris and the protist Tetrahymena thermophila to examine whether predation pressure can initiate the formation of colonies. We find that (1) predators and predator exoproducts promote colony formation, (2) higher predator densities cause more colonies to form and (3) colony formation in this system is facultative. Our results suggest that predators could be a key force promoting the formation of multicellular groups, and thus may be important in understanding the first stages of the evolution of multicellularity.
\end{abstract}

\section{Introduction}

The evolution of multicellularity is one of the major evolutionary transitions and has led to the evolution of the dominant organisms on earth; animals, plants and fungi. Multicellularity has evolved over 25 times across the tree of life (Grosberg \& Strathmann 2007) with species ranging from simple organisms like photosynthetic cyanobacteria, to the most complex metazoans with hundreds of different cell types. During the evolution of a multicellular organism, individual cells cooperate to form a group comprised of many cells. Why they would do this is not clear, because the formation of groups could have fitness costs, such as increased competition for resources (Lurling \& Van Donk 2000). 
One theory is that cells may group together to defend themselves against predation (Grosberg \& Strathmann 2007; Stanley 1973). The benefit of being part of a group could therefore outweigh any costs such as increased resource competition. The formation of colonies in the presence of predators has been experimentally shown in several species of algae (Table 1), and an analogous type of predator defence can be seen in the formation of social groups in animals (Bourke 2014; Shultz et al. 2011; Korb et al. 2012; Pike et al. 2007).

Recent experimental studies have shown that colony formation can be favoured by factors other than predation (Ratcliff et al. 2012; Ratcliff et al. 2013; Hammerschmidt et al. 2014). Ratcliff et al. (2013) showed that both Chlamydomonas reinhardtii and Saccharomyces cerevisiae formed obligate multicellular clusters under intense settling selection (Ratcliff et al. 2013; Ratcliff et al. 2012), and the maintenance of a Pseudomonas fluorescens multicellular phenotype has also been shown in laboratory conditions (Hammerschmidt et al. 2014). These studies highlight that many factors could have been important for providing the initial benefit of multicellularity, but in this study we focus on predation.

The previous work on multicellular group formation in algae in the presence of predators needs expanding in a number of ways. Firstly, many studies have focused on the number of colonies formed in the presence of predators, rather than the proportion of cells in colonies (Table 1). The number of colonies is confounded with population growth, and so does not necessarily reflect whether cells are more likely to form groups in the presence of predators. Secondly, several studies have been anecdotal, lacking formal statistical 
analyses (Boraas et al. 1998), or have not included appropriate controls (for example, treatments to elucidate the specific cause of colony formation) (Yang et al. 2006; Yang \& Li 2007; Boraas et al. 1998). Furthermore, many previous studies have not tested explicity whether colonies persisted over time (i.e. were obligate) or whether populations reverted back to being unicellular (i.e. were facultative) (Lurling et al. 2000; Lurling et al. 1997; Mayeli et al. 2005; Long et al. 2007). This distinction is important, because the formation of a heritable colony-forming phenotype is a key step towards the evolution of an obligate multicellular organism.

In this study, we use a potential predator-prey system, the green alga Chlorella vulgaris and the protist Tetrahymena thermophila, to test whether the presence of a predator promotes colony formation. If colony formation is a defensive response to predation, we would expect to see an increase in the proportion of cells in colonies when predators are present, a stronger response when the density of predators is high and a response to cues of predator presence, as well as actual predators. We test these predictions by examining: (1) whether T. thermophila can induce colony formation in C. vulgaris; (2) if $T$. thermophila exoproducts were sufficient for this response; (3) if T. thermophila density influenced the strength of the colony induction; and (4) whether colony formation was obligate or facultative. 
Table 1: Examples of colony induction experiments in species of green algae and bacteria. indicates that colony formation in the presence of predators was observed. $\boldsymbol{X}$ indicates that no colony formation was observed. - indicates that the test was not performed.

\begin{tabular}{|c|c|c|c|c|c|c|}
\hline \multirow[b]{2}{*}{$\begin{array}{l}\text { Algae/bacteria } \\
\text { species }\end{array}$} & \multirow[b]{2}{*}{ Predator species } & \multirow[b]{2}{*}{$\begin{array}{l}\text { Measure(s) of } \\
\text { colony induction }\end{array}$} & \multicolumn{3}{|c|}{ Colony induction? } & \multirow[b]{2}{*}{ Reference } \\
\hline & & & $\begin{array}{l}\text { Live } \\
\text { predators }\end{array}$ & $\begin{array}{l}\text { Predator } \\
\text { exoproducts }\end{array}$ & $\begin{array}{l}\text { Predator }+ \\
\text { algae } \\
\text { exoproducts }\end{array}$ & \\
\hline Cyanobium sp. & Ochromonas sp. & Colony abundance & $\checkmark$ & - & $\overline{-}$ & $\begin{array}{l}\text { (Jezberová } \\
\& \\
\text { Komárková } \\
\text { 2007) } \\
\end{array}$ \\
\hline \multirow{3}{*}{$\begin{array}{l}\text { Microcystis } \\
\text { aeruginosa }\end{array}$} & Ochromonas sp. & Colony abundance & - & - & $\checkmark$ & $\begin{array}{l}\text { (Yang et } \\
\text { al. 2009) }\end{array}$ \\
\hline & Daphnia magna & $\begin{array}{l}\text { Number of cells } \\
\text { per colony, mean } \\
\text { particle volume }\end{array}$ & - & - & $\checkmark$ & $\begin{array}{l}\text { (Ha et al. } \\
2004)\end{array}$ \\
\hline & Moina macrocopa & $\begin{array}{l}\text { Number of cells } \\
\text { per colony, mean } \\
\text { particle volume }\end{array}$ & - & - & $\checkmark$ & $\begin{array}{l}\text { (Ha et al. } \\
2004)\end{array}$ \\
\hline Phaeocystis globosa & $\begin{array}{l}\text { Gyrodinium } \\
\text { dominans }\end{array}$ & Colonies $\mathrm{ml}^{-1}$ & $\checkmark$ & - & - & $\begin{array}{l}\text { (Jakobsen } \\
\& \quad \text { Tang } \\
\text { 2002) } \\
\end{array}$ \\
\hline Phaeocystis & Euplotes & $\begin{array}{l}\% \text { of total cells in } \\
\text { colonies }\end{array}$ & $\checkmark$ & - & - & $\begin{array}{l}\text { (Long et al. } \\
2007 \text { ) }\end{array}$ \\
\hline \multirow{3}{*}{ Scenedesmus acutus } & Daphnia pulex & Cells per colony & - & $\checkmark$ & - & $\begin{array}{l}\text { (Lurling \& } \\
\text { Van Donk } \\
\text { 1996) }\end{array}$ \\
\hline & Daphnia galeata & $\begin{array}{l}\text { Proportion of cells } \\
\text { in colonies of } \\
\text { different sizes, } \\
\text { number of cells per } \\
\text { colony }\end{array}$ & $\checkmark$ & $\checkmark$ & - & $\begin{array}{l}\text { (Lurling et } \\
\text { al. 1997) }\end{array}$ \\
\hline & Daphnia magna & $\begin{array}{l}\text { Proportion of cells } \\
\text { in colonies of } \\
\text { different sizes, } \\
\text { mean particle } \\
\text { volume }\end{array}$ & - & $\checkmark$ & - & $\begin{array}{l}\text { (Lampert et } \\
\text { al. 1994) }\end{array}$ \\
\hline $\begin{array}{l}\text { Scenedesmus } \\
\text { subspicatus }\end{array}$ & Daphnia magna & $\begin{array}{l}\text { Cells per colony, } \\
\text { mean particle } \\
\text { volume }\end{array}$ & $\checkmark$ & - & $x$ & $\begin{array}{l}\text { (Lurling \& } \\
\text { Van Donk } \\
\text { 1997) }\end{array}$ \\
\hline $\begin{array}{l}\text { Scenedesmus } \\
\text { obliquus }\end{array}$ & Daphnia magna & $\begin{array}{l}\text { Cells per colony, } \\
\text { mean particle } \\
\text { volume }\end{array}$ & $\checkmark$ & - & $\checkmark$ & $\begin{array}{l}\text { (Lurling \& } \\
\text { Van Donk } \\
\text { 1997) }\end{array}$ \\
\hline $\begin{array}{l}\text { Scenedesmus } \\
\text { dimorphus }\end{array}$ & Moina macrocopa & $\begin{array}{l}\text { Number of cells } \\
\text { per colony, mean } \\
\text { particle volume }\end{array}$ & - & - & $\checkmark$ & $\begin{array}{l}\text { (Ha et al. } \\
2004)\end{array}$ \\
\hline Chlorella vulgaris & $\begin{array}{l}\text { Ochromonas } \\
\text { vallescia }\end{array}$ & - & $\checkmark$ & - & - & $\begin{array}{l}\text { (Boraas et } \\
\text { al. 1998) }\end{array}$ \\
\hline $\begin{array}{l}\text { Chlamydomonas } \\
\text { reinhardtii }\end{array}$ & Brachionus & $\begin{array}{l}\text { \% single cells, } \\
\text { mean clump size }\end{array}$ & $\checkmark$ & - & - & $\begin{array}{l}\text { (Becks et } \\
\text { al. 2010) }\end{array}$ \\
\hline Flectobacillus sp. & Ochromonas sp. & $\begin{array}{l}\text { cell volume, cell } \\
\text { length/width, cell } \\
\text { number, \% inedible } \\
\text { morphotypes }\end{array}$ & $\checkmark$ & $\checkmark$ & - & $\begin{array}{l}\text { (Corno \& } \\
\text { Jurgens } \\
2006)\end{array}$ \\
\hline $\begin{array}{l}\text { Comamonas } \\
\text { acidovorans }\end{array}$ & Ochromonas sp. & $\begin{array}{l}\text { \% single cells, } \\
\text { mean cell volume }\end{array}$ & $\checkmark$ & - & - & $\begin{array}{l}\text { (Hahn et } \\
\text { al. 1999) }\end{array}$ \\
\hline
\end{tabular}




\section{Methods}

In all experiments, we used the green algae species Chlorella vulgaris and the ciliate protist Tetrahymena thermophila. We purchased both as axenic cultures from the Culture Collection of Algae and Protozoa (CCAP); strain numbers 211/11B and 1630/1M respectively. We chose $C$. vulgaris for this study because it has been previously been shown to form colonies (Boraas et al. 1998) and we wanted to expand on this observation with statistical analysis and further tests of the factors causing colony formation. We chose $T$. thermophila because it is easy to grow in axenic culture and is common in freshwater environments where $C$. vulgaris can be found. We grew stock cultures of $C$. vulgaris at room temperature in $300 \mathrm{ml}$ of Bolds Basal Media (Bolds) and kept them on a shaker to prevent sedimentation. We grew T. thermophila cultures at room temperature in $10 \mathrm{ml}$ of Proteose Peptone Yeast Media (PPY) and subcultured stocks every week by transferring $100 \mu 1$ to $10 \mathrm{ml}$ of fresh PPY media.

To get independent readings each day for five days, we set up three replicates of each treatment (described in detail for each experiment below) and repeated these five times (three replicates of each treatment each day for five days). We then randomised these treatments in 24-well plates $(1 \mathrm{ml}$ volume per well). We therefore only measured each microcosm once, so that all measurements on consecutive days would be independent. We kept the plates on a microplate shaker in a $20^{\circ} \mathrm{C}$ incubator with a $16 / 8$-light/dark cycle.

We produced supernatants of: (1) predator only culture; (2) algae only culture; and (3) algae and predator culture. We produced the supernatant of predator culture by filtering $T$. thermophila in PPY media (400000 cells $/ \mathrm{ml}$ ) through a $0.22 \mu \mathrm{m}$ syringe filter, to remove 
all T. thermophila cells, leaving just a supernatant of exoproducts and PPY media. We produced the algae supernatant using the same method, but with C. vulgaris in Bolds media. We produced the algae and predator supernatant by adding T. thermophila in PPY to $C$. vulgaris culture and then leaving this mixture for one hour. We then filtered this through a $0.22 \mu \mathrm{m}$ syringe filter to remove all cells, leaving only exoproducts and media. These different supernatants are referred to throughout as: 'predator supernatant' (supernatant of $T$. thermophila culture), 'algae supernatant' (supernatant of C. vulgaris culture), and 'algae and predator supernatant' (supernatant of $T$. thermophila and $C$. vulgaris culture).

In order to quantify the effect of predation on the algal populations, we captured images of the experimental cultures using a VisiCam on an inverted light microscope and processed the images using ImageJ software. In all experiments, we took one photograph of each relevant (e.g. day one, two, three etc.) microcosm at 20x magnification, focusing on a random area of the well. We then used ImageJ software to manually count unicells and colonies in each photograph, using the CellCounter plugin. We defined three or more cells with cell walls touching to be colonies. Where possible, we estimated the size of each colony, and calculated mean colony size (although for some colonies this was not possible due to poor image quality). If cells appeared to be paired, we counted them as two unicells.

\section{Experiment 1: Do predators induce colony formation?}

We tested whether the timing and number of times that $T$. thermophila was added to algal populations had any influence on colony formation. The experiment had three treatments:

(1) the addition of $T$. thermophila to $C$. vulgaris on day one only (p1), (2) the addition of 
T. thermophila to C.vulgaris every day for five days (p1-5) and, (3) the addition of $T$. thermophila to C. vulgaris on day three (p3). As a control we used C. vulgaris in PPY media. We replicated each treatment 15 times, giving a total of $4 \times 15=60$ microcosms, which we randomised in 24-well plates.

\section{Experiment 2: Do predator exoproducts induce colony formation?}

We tested whether colony formation occurred in the presence of $T$. thermophila exoproducts. The experiment had four treatments: (1) the addition of $50 \mu \mathrm{l}$ T. thermophila (roughly 10000 individuals) in PPY media to $950 \mu \mathrm{l}$. vulgaris, (2) addition of $50 \mu \mathrm{l}$. thermophila supernatant to $950 \mu \mathrm{l}$. vulgaris, (3) addition of $T$. thermophila and $C$. vulgaris supernatant to $950 \mu \mathrm{l}$ C. vulgaris and (4) addition of $50 \mu \mathrm{l} C$. vulgaris supernatant to $950 \mu \mathrm{l}$ C. vulgaris. We added the live predator treatment, as we wanted to control for potential differences with Experiment 1. We used two controls; (1) $1 \mathrm{ml} \mathrm{C}$. vulgaris in Bolds media and (2) $950 \mu \mathrm{l} \mathrm{C}$. vulgaris with $50 \mu \mathrm{PPY}$ media (to control for the increased growth in high nutrient PPY media). We replicated each treatment 15 times, giving a total of $5 \times 15=75$ microcosms, which we randomised in 24 -well plates.

\section{Experiment 3: Does predator density influence colony formation?}

We tested whether T. thermophila density affected the strength of colony formation in $C$. vulgaris. The experiment had three treatments: (1) low density of T. thermophila (11000 cells/ml), (2) medium density of $T$. thermophila (31000 cells/ml) and (3) high density of T. thermophila (1000000 cells $/ \mathrm{ml})$, all of which were added to C. vulgaris culture. As a control we used $C$. vulgaris in PPY media. We replicated each treatment 15 times, giving a total of $6 \times 15=90$ microcosms, which we randomised in 24 -well plates. 


\section{Experiment 4: Is colony formation facultative?}

This experiment was an extension of Experiment 1 . We tested whether colony formation in C. vulgaris is obligate or facultative. We kept Experiment 1 plates in the incubator for an extra 15 days without any additional T. thermophila, and we transferred to fresh media on day 10. On day 20, we took photographs of the microcosms and quantified colony formation using the same protocol described above.

\section{Statistical methods}

We used R version 2.14.1 for statistical analysis. We used generalized linear models and specified error structures as quasipoisson (for number of colonies and total number of cells) and quasibinomial (for proportion of cells in colonies) to account for overdispersion in the data, and Gaussian (for colony size). In all analyses, the treatment (e.g. density of predators) was used as the explanatory variable, and the response variable was either the number of colonies, the proportion of cells in colonies, mean colony size or total number of algal cells. To test for differences between each treatment, we used Tukey honest significant differences (Tukey HSD). We only tested for differences between means on relevant days, for example the day after predators had been added or on the final day of the experiment, as we were interested in the immediate effect of predators or predator exoproducts on colony formation and not the overall population dynamics of algal cultures.

\section{Results}

Experiment 1: Do predators induce colony formation? 

a) unicellular C. vulgaris

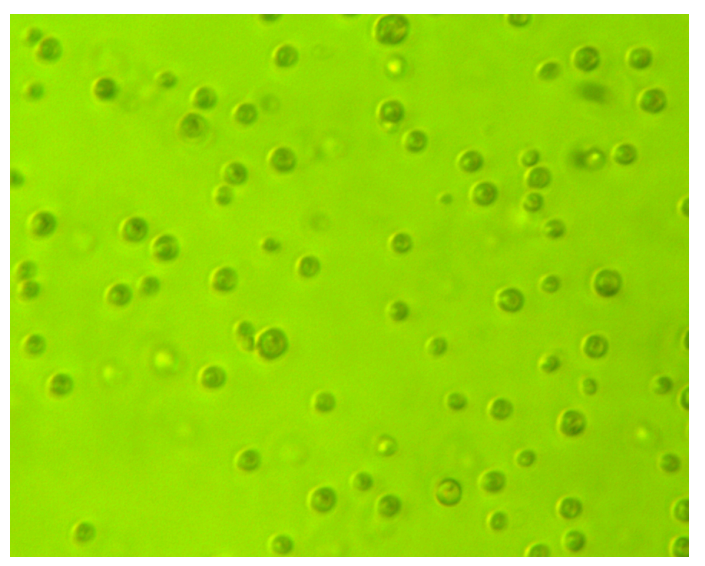

b) C. vulgaris + T. thermophila

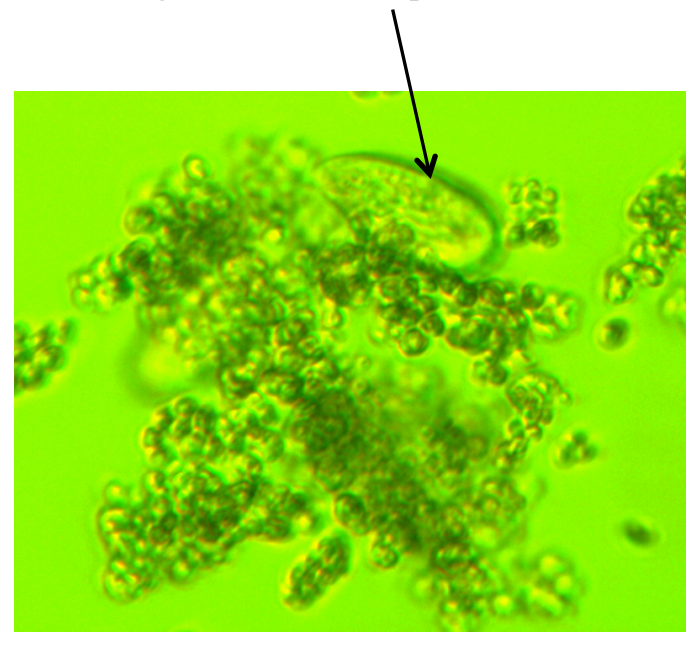

Figure 1: Examples of $\boldsymbol{C}$. vulgaris and T. thermophila cultures. Image a) shows $C$. vulgaris unicells when predators are absent. Image b) shows the formation of colonies when predators are added (a particularly large colony is shown for illustrative purposes). An individual T. thermophila protist can be seen in image b), indicated with an arrow. Both images were taken using VisiCam software at 40x magnification.

a)

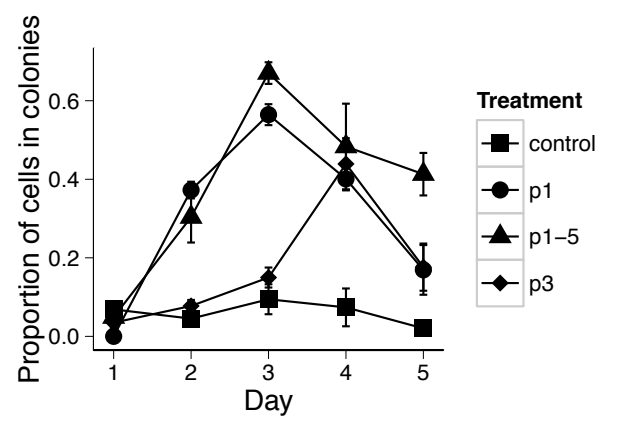

c)

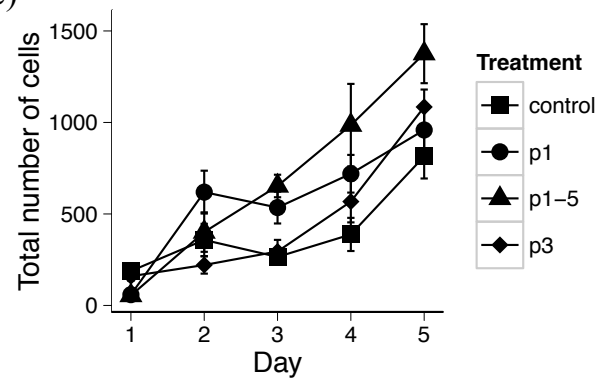

b)

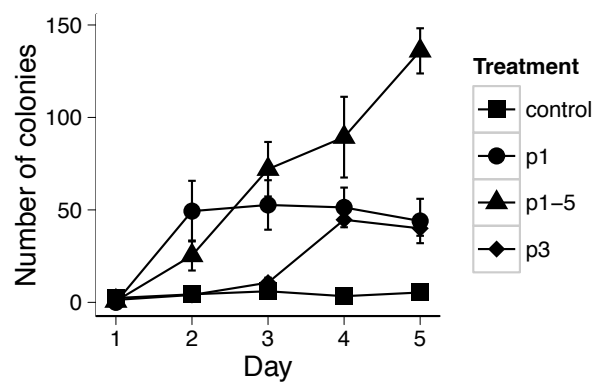

Figure 2: Predators induce colony formation. Plots showing a) the proportion of cells in colonies, b) the number of colonies, c) total number of algal cells for all treatments. Treatments were $\mathrm{p} 1$ : predators added on day one, $\mathrm{p} 1-5$ : predators added every day, $\mathrm{p} 3$ : predators added on day 
three, control: no predators added. $\mathrm{N}=3$ for each treatment on each day. Means and standard errors are shown.

By day three, the treatments where predators had been added on day one ( $\mathrm{p} 1$ and p1-5) had significantly higher proportion of cells in colonies than treatments where predators had not been added (control and $\mathrm{p} 3),(\mathrm{p} 1: \mathrm{p}=0.001 ; \mathrm{p} 1-5: \mathrm{p}=0.001 ; \mathrm{p} 3: \mathrm{p}=0.21 ;$ overall difference between all treatments: $F=57.33, \mathrm{df}=3, \mathrm{p}=0.001$ ) (Fig. $1 \& 2 \mathrm{a}$ ). On day five, only the treatment when predators had been continually added (p1-5) had a significantly higher proportion of cells in colonies compared to the control $(\mathrm{p} 1: \mathrm{p}=0.09 ; \mathrm{p} 1-5: \mathrm{p}=$ $0.01 ; \mathrm{p} 3: \mathrm{p}=0.08$; overall difference between all treatments: $\mathrm{F}=10.78, \mathrm{df}=3, \mathrm{p}=0.003$ ) and compared to when predators had only been added on day one or three $(\mathrm{F}=14.11, \mathrm{df}=$ $1, \mathrm{p}=0.007)$

We found a similar pattern for the number of colonies (Fig. 2b). By day three, treatments where predators had been added on day one (p1 and p1-5) had a significantly more colonies than treatments where predators had not been added (control and p3), $(\mathrm{p} 1: \mathrm{p}=$ $0.007 ; \mathrm{p} 1-5: \mathrm{p}=0.003 ; \mathrm{p} 3: \mathrm{p}=0.44 ;$ overall difference between all treatments: $\mathrm{F}=16.81$, $\mathrm{df}=3, \mathrm{p}=0.001)$. On day five, all treatments where predators had at some point been added had significantly more colonies compared to the control where predators had not been added $(p 1: p=0.009 ; p 1-5: p=0.001 ; p 3: p=0.01 ;$ overall difference between all treatments: $\mathrm{F}=31.05, \mathrm{df}=3, \mathrm{p}=0.001)$. When we added predators continually $(\mathrm{p} 1-5)$, this led to significantly more colonies than when we just added predators on day one or three (p1 and p3) and all other treatments (TukeyHSD, p = 0.001). In contrast, we found that neither mean colony size $(\mathrm{glm}, \mathrm{df}=3, \mathrm{~F}=2.51, \mathrm{p}=0.07)$ or the total number of algal cells significantly varied depending on whether predators were added or not (glm, $\mathrm{df}=3$, $\mathrm{F}=0.45, \mathrm{p}=0.72)($ Fig. $2 \mathrm{c})$ 


\section{Experiment 2: do predator exoproducts induce colony formation?}

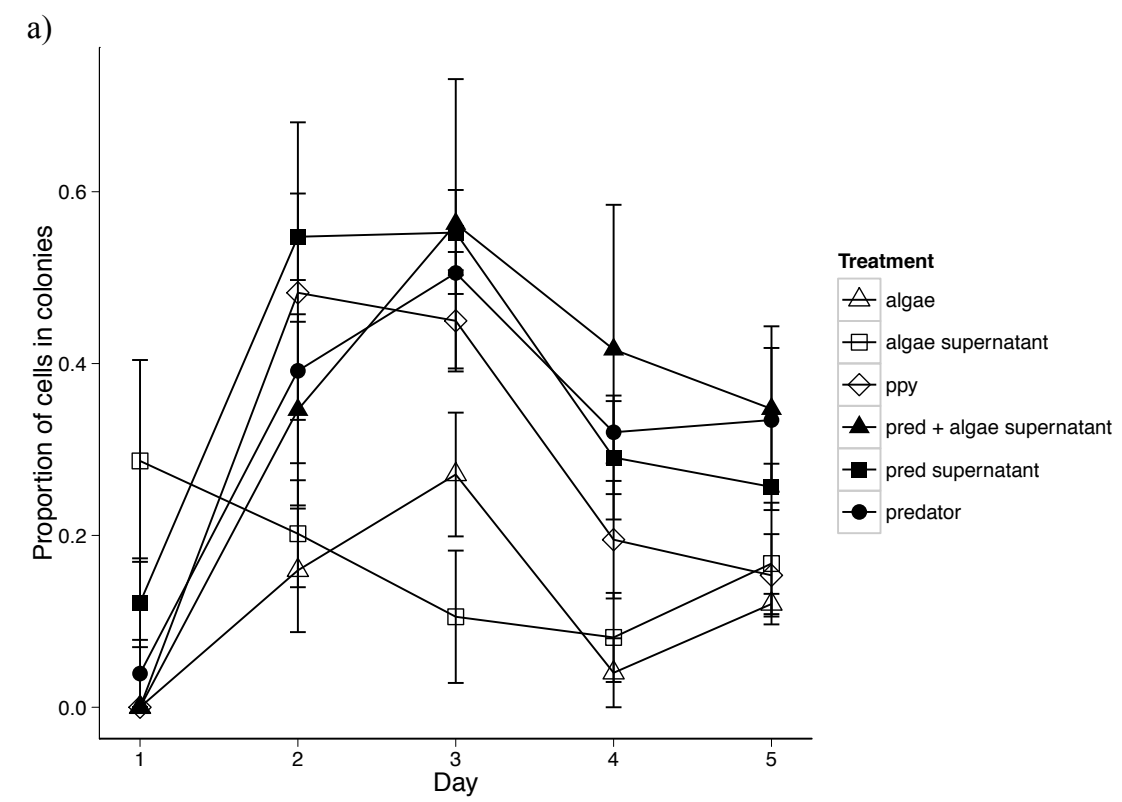

b)

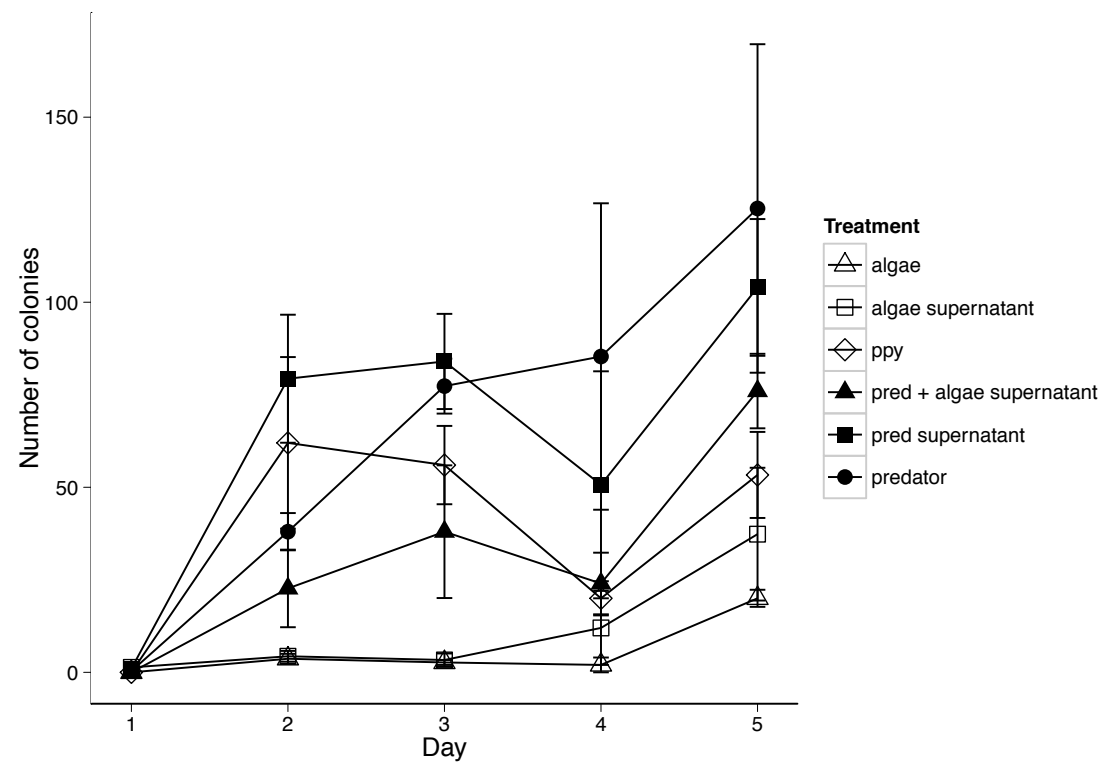

Figure 3: Predator exoproducts induce colony formation. a) the proportion of cells in colonies and b) the number of colonies. 'Predator' related treatments (predators, predator supernatant and predator + algae supernatant) are shown with filled shapes, and 'control' treatments (algae, algae supernatant and algae + PPY media) are shown with open shapes. $\mathrm{N}=3$ for each treatment on each day. Means and standard error bars are shown.

We found no difference in the proportion of cells in colonies between any treatments on day one (glm, TukeyHSD, $\mathrm{p}=>0.05$ between all treatments). By day three we found that 'predator related' treatments (pred + algae supernatant, pred supernatant and live 
predators) did not have a significantly higher proportion of cells in colonies compared to control treatments (algae, ppy and algae supernatant) $(\mathrm{glm}, \mathrm{F}=4.42$, $\mathrm{df}=1, \mathrm{p}=0.05)$ (Fig. 3a). However, on day five, we found that 'predator related' treatments had significantly higher proportion of cells in colonies than control treatments ( $g 1 m, F=10.16$, $\mathrm{df}=1, \mathrm{p}=0.006)$. The same results where obtained when we compared the "predator related' treatments to just the ppy control on day five (day 5: $\mathrm{F}=5.61, \mathrm{df}=1, \mathrm{p}=0.04$ ), but not day three (day $3: \mathrm{F}=1.42, \mathrm{df}=1, \mathrm{p}=0.26$ ). Considering the number of colonies, we found that 'predator related' treatments had significantly more colonies compared to the control treatments on both days three ( $\mathrm{glm}, \mathrm{F}=8.89, \mathrm{df}=1, \mathrm{p}=0.009)$ and five $(\mathrm{glm}$, $\mathrm{F}=15.23, \mathrm{df}=1, \mathrm{p}=0.001)($ Fig. $3 b)$

We found no significant difference in mean colony size between supernatant treatments and the control $(\mathrm{glm}, \mathrm{F}=0.53, \mathrm{~N}=12, \mathrm{p}=0.75)$. We ran this analysis on a subset of the full dataset with day one eliminated, because we did not have data on mean colony size for that day.

\section{Experiment 3: does predator density influence colony formation?}

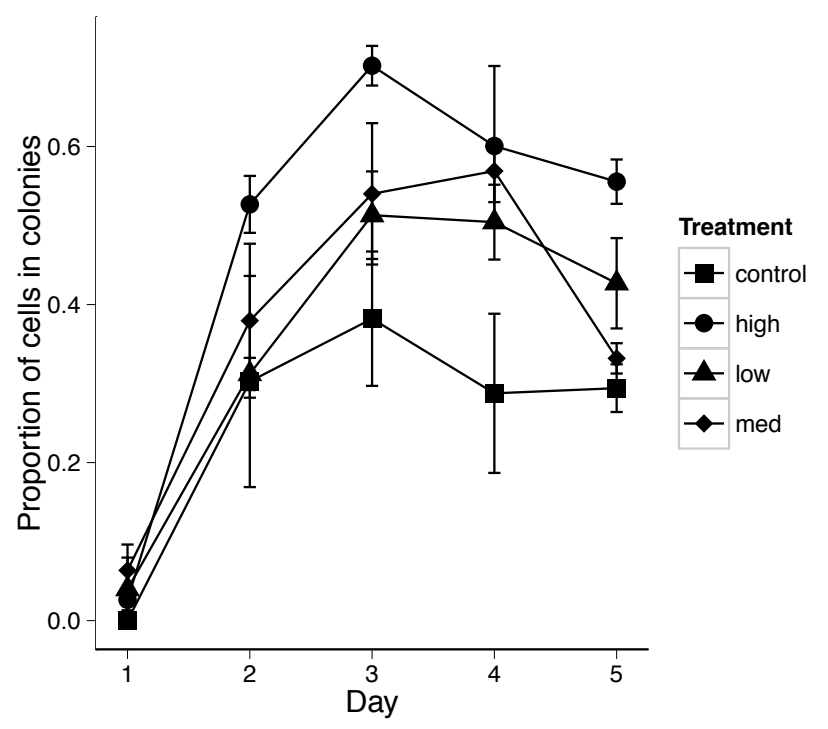


Figure 4: Predator density influences the proportion of cells in colonies. The three treatments were: low density of $T$. thermophila $(11000 \mathrm{cells} / \mathrm{ml})$, medium density of $T$. thermophila $(31000$ cells $/ \mathrm{ml})$ and high density of $T$. thermophila $(1000000 \mathrm{cells} / \mathrm{ml})$ and the control was $C$. vulgaris in PPY media. $\mathrm{N}=3$ for each treatment on each day. Means and standard errors are shown.

There was a significantly higher proportion of cells in colonies when predator density was higher, examining days three $(\mathrm{glm}, \mathrm{F}=9.88$, $\mathrm{df}=1, \mathrm{p}=0.01)$, four $(\mathrm{glm}, \mathrm{F}=10.76$, $\mathrm{df}=$ $1, p=0.008)$ and five ( $g \operatorname{lm}, F=10.19, d f=1, p=0.01)($ Fig. 4). In contrast, there was no significant difference in mean colony size between control and treatments ( $\mathrm{glm}, \mathrm{F}=0.25$, $\mathrm{df}=3, \mathrm{p}=0.86)$

\section{Experiment 4: is colony formation facultative?}
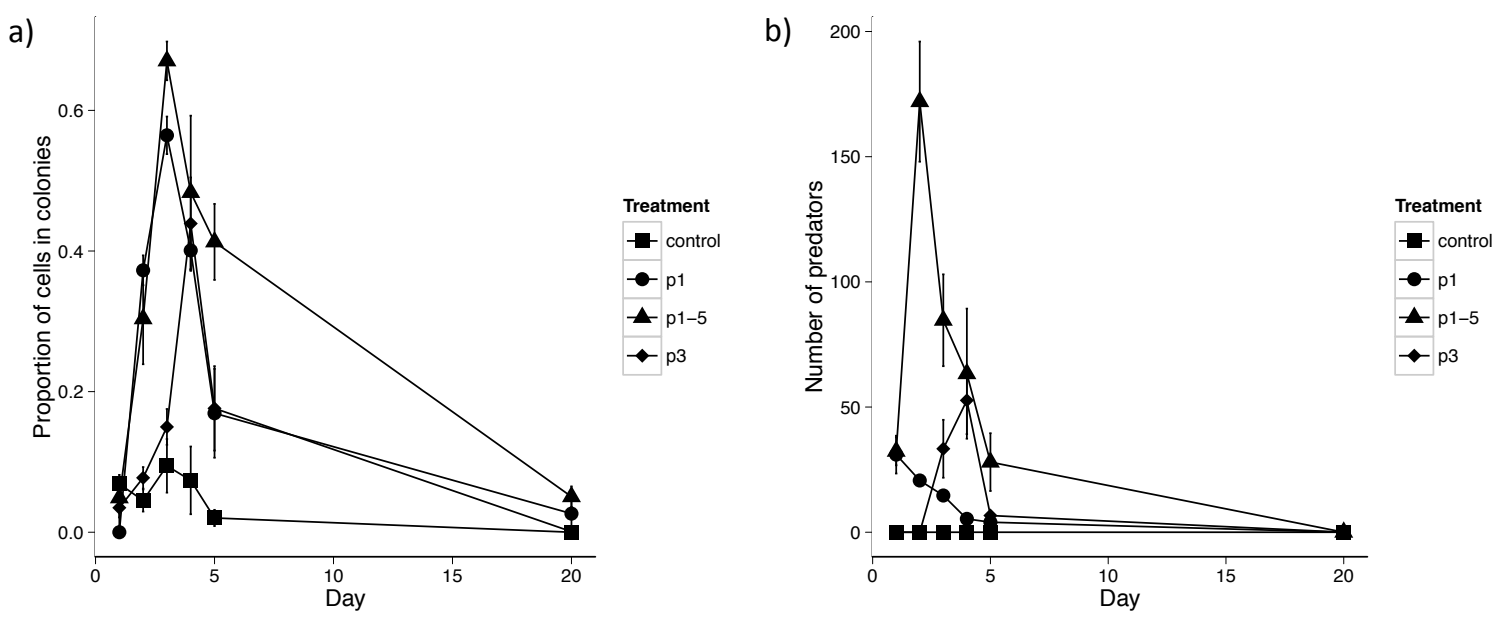

Figure 5: Colony formation is a facultative trait. a) The proportion of cells in colonies and b) the number of live, mobile predators (per field of view at x10 magnification) for all treatments (p1: predators added on day one, p1-5: predators added every day, p3: predators added on day three, control: no predators added) over the course of 20 days. a) Days 1-5 show the same data as in Figure 2a, from Experiment 1. $\mathrm{N}=3$ for each treatment on each day. Means and standard errors are shown.

By day 20, we found no difference between any of the treatments where predators had been added compared to the treatment where no predators had been added ( $p 1: p=1.00$; $\mathrm{p} 1-5: \mathrm{p}=1.00 ; \mathrm{p} 3: \mathrm{p}=1.00 ;$ overall difference between all treatments: $\mathrm{F}=6.19, \mathrm{df}=3, \mathrm{p}$ $=0.02)($ Fig. 5a). 


\section{Discussion}

We found that; (1) the presence of the protist predator T. thermophila promotes colony formation in the algae C. vulgaris (Fig. $1 \& 2$ ); (2) supernatants taken from predator cultures and predator/algae cultures are able to promote colony formation (Fig. 3); (3) higher predator densities cause more cells to form colonies (Fig. 4); and (4) colony formation in this system is facultative, with populations reverting to being predominantly unicellular after 20 days (Fig 5).

This is the first study to show that $T$. thermophila can promote colony formation in $C$. vulgaris (Fig. 2), and that predator and predator/algae exoproducts are sufficient to induce this response (Fig. 3). It has previously been observed that the presence of predators causes colony formation in C. vulgaris (Boraas et al. 1998). However, we have shown experimentally that $C$. vulgaris forms colonies not only in response to live predators, but also predator and predator/algae exoproducts and that a higher density of predators causes more colonies to form (Fig. $2 \& 4$ ). Previous studies have also observed that predator exoproducts promote colony formation in other green algae (Van Donk et al. 2011). Our results are consistent with the hypothesis that colony formation is a defensive response to the presence of predators and suggests that predation pressure could be important for the evolution of cooperative multicellular groups.

Notably, even when predators are added continually to algal cultures for five days (treatment p1-5), we see a decrease in the proportion of cells in colonies after day three (Fig. 2a). This suggests that after an initial strong response to predators (days one to 
three), the colonies begin to break up (leading to a lower proportion of cells in colonies). This could be due to a fluctuation in predator numbers, and thus intensity of predation pressure, or because colony formation is costly and nutrients have been depleted. Alternatively, it may be that this slight decrease in the proportion of cells in colonies is due to natural variation and had we measured for longer we would have seen recovery.

Our results cannot exclude the possibility that the increased proportion of cells in colonies is a response to selection for colonies, as opposed to phenotypic plasticity. In our experimental setup, C. vulgaris has a doubling time of roughly 4 hours, so it is possible that over five days (roughly 30 generations) we are seeing experimental evolution of multicellular groups. However, experimental evolution in Chlamydomonas (Ratcliff et al. 2013) has shown that multicellular clusters take over 200 days to evolve $(>300$ generations), even under intense selection regimes, so it is unlikely that we are seeing evolution over five days.

We found that the presence of predators had no significant influence on mean colony size (Fig. 2c). One possible explanation is that there is an optimum colony size, for a given environment. For example, if competition for resources led to an increasing cost of forming larger colonies (Yang et al. 2009), then this would have to be traded off against any increased ability to defend against predators (Lurling \& Van Donk 2000). Some predators of algae are size-selective (Long et al. 2007), and so the benefits of increased colony size could also asymptote with colony size.

We observed that the total number of $C$. vulgaris cells did not differ when $T$. thermophila were present or not (Fig. 2d). There are at least three possible explanations for this. First, 
the predator densities that we used, whilst sufficient to trigger colony formation, were not sufficient to impact on population growth. Second, the increased colony formation was able to prevent appreciable decrease in the total number of algal cells through increased defence, and thus survivorship. Third, that $T$. thermophila is a poor predator of $C$. vulgaris. The first and third of these possibilities would indicate a generic response by $C$. vulgaris to potential predators in its local environment, rather than the specific danger of predation.

We have shown that in our experimental setup colony formation in C. vulgaris is facultative, with populations reverting back to being unicellular when predators are absent (Fig. 5). In contrast to our result, Boraas et al. (1998) found that C. vulgaris colonies persisted for several months even after predators were removed, which could be because they used a different predator, the flagellate protist Ochromonas vallescia. Another difference that could have affected the degree of colony formation is that their experimental system used $500 \mathrm{ml}$ chemostat cultures, compared to ours which used smaller volumes. Facultative colony formation may be favoured over the formation of obligate colonies, because it allows algae to have a flexible response to different types of predators (Van Donk et al. 2011), particularly if the benefit of colony formation only outweighs the cost in specific environments. Our observations of $C$. vulgaris colonies suggest that the cells are held together with a gelatinous extracellular matrix, which could indicate that colonies are forming via aggregation, and not through incomplete cell division (contrasting with the result of Boraas et al. 1998). The next steps will be investigating the costs and benefits of colony formation in this system, and clarifying how multicellular groups form, so we can fully understand the role predators might play in the evolution of multicellularity. 


\title{
Chapter 4: The evolution of bacterial symbiosis
}

\begin{abstract}
Symbiosis between bacteria and their hosts is a potential between-species major evolutionary transition in individuality. Bacterial symbionts are found in a diverse range of species, but the degree to which they benefit their hosts varies. One major suggestion to explain this variation has been transmission route, with vertical transmission of clonal symbionts leading to higher levels of cooperation than horizontal transmission. However, it has also been suggested that horizontal transmission may be particularly beneficial in changeable or stressful environments. Here, we test the hypothesis that both transmission mode and environment determine how much symbionts should benefit their hosts using a phylogenetically controlled comparative analysis of studies measuring host fitness on removal of their symbiont(s). We find (1) that horizontally transmitted symbionts are relatively more beneficial in stressful versus unstressful environments, (2) that nutritional symbioses are more beneficial to hosts than defensive ones, and (3) there is no difference in host benefit between obligate and facultative symbionts. Our results show that to understand the evolution of symbiosis we need to consider multiple aspects of transmission mode and ecology.
\end{abstract}

\section{Introduction}

The evolution of symbiosis represents a between-species major evolutionary transition. It led to the origins of mitochondria and chloroplasts through endosymbiosis, and thus the evolution of the eukaryotic cell (Keeling 2010) and underpins ecologically important species such as corals. Bacterial symbionts are found frequently in nature, particularly in insects (Sachs et al. 2011; Moran et al. 2008) and they commonly provide nutrition to the 
host (Douglas 1998b) or are involved in defence, by producing useful compounds such as antibiotics (Kaltenpoth 2009) or by protecting against parasites (Oliver et al. 2003).

Although symbiosis is defined as any long-lasting interaction where both partners receive a benefit (Herre et al. 1999; Sachs et al. 2011), the degree of benefit to the host can vary dramatically (Sachs \& Wilcox 2006; Hosokawa et al. 2010). For example, Buchnera bacteria, found in aphids, are extremely cooperative nutritional symbionts that provide their hosts with essential amino acids (Douglas 1996). On the other hand, rhizobia bacteria vary in how cooperative they are, and non-cooperative cheats can arise in natural populations (Sachs et al. 2010). The problem is understanding why some symbioses seem exclusively cooperative, to the extent that the host becomes completely dependent on the symbiont, and vice versa, whereas others do not.

It has been suggested that transmission route could explain variation in symbiont cooperation (Douglas 1998a; Ebert 2013). Symbionts will be selected to maximize their fitness, as will hosts, and so cooperation should be most favoured when maximizing the symbionts' fitness is aligned with maximizing the host's reproductive output (Bourke 2011). Vertical transmission of symbionts, leading to the fitness interests of the host and symbiont becoming aligned, has been suggested to lead to more beneficial symbionts (Herre et al. 1999; Frank 1996b; Yamamura 1993). Conversely, horizontal transmission, resulting in multiple symbiont genotypes coexisting within a host will lead to higher levels of host-symbiont conflict, and the evolution of less beneficial symbiotic partners (Frank 1996b). However, even with horizontal transmission, hosts may be able to select the most cooperative strains of symbionts, leading to higher than predicted levels of cooperation (Aanen et al. 2009; Kiers et al. 2003). 
However, it has been suggested that horizontal transmission may be particularly beneficial for hosts that experience unpredictable or variable environments, as it exposes them to a much wider pool of potential symbiotic partners (Henry et al. 2013; Douglas 1998a) and allows uptake of novel traits (Oliver et al. 2010). For example, corals can acquire diverse photosynthetic symbionts from the surrounding water, and particular groups of these symbionts have different characteristics, such as certain strains of Symbiodinium conferring higher thermal tolerance for their hosts than others (Mieog et al. 2009).

There have been few experimental (Bull et al. 1991; Clayton \& Tompkins 1994; Pagan et al. 2014; Sachs \& Wilcox 2006) and no comparative tests of the hypothesis that vertical transmission generally leads to higher cooperation, and that horizontally transmitted symbionts may be particularly beneficial in stressful environments. Here, we test the effect of transmission route (either strictly vertical or some horizontal transmission) on cooperation between bacterial symbionts and hosts, and the effect that the environment (stressful or not) has on this relationship, using a phylogenetically controlled comparative analysis. We focus on bacterial symbionts because: (1) they have given rise to the most extreme examples of symbiosis; mitochondria and chloroplasts; (2) they are numerous and have a diverse range of hosts and; (3) there are examples of vertically and horizontally transmitted bacterial symbionts. We calculate the host fitness change when symbionts are removed from hosts.

\section{Methods}


We searched for papers that measured host fitness with and without their bacterial symbionts, both from experimental manipulation and from natural populations. We found papers by (1) searching combinations of the following key words using Papers 2 (covering Scopus, Web of Knowledge, JSTOR, Google Scholar and PubMed): bacteria, symbiosis, mutualism, fitness, removal, elimination, fecundity, dependence, obligate, facultative, aposymbiosis, (2) forward and backward searches on papers and (3) searching key reviews of symbiosis including Moran et al. (2008), Moya et al. (2008), Sachs et al. (2011), Bright \& Bulgheresi (2010), Baumann (2005), Oliver et al. (2010).

We eliminated any papers that (1) did not include fitness measurements with and without bacterial symbionts; (2) where the PDF was unavailable or not available in English; (3) were not studying a bacterial symbiont and (4) studies involving the introduction of a novel symbiont to a naïve host. Where a paper provided observational evidence or raw data were not included in the paper, we contacted the corresponding author.

After the elimination criteria (above) had been applied, the literature search returned over 200 papers directly related to the study question. The final database included data from 58 studies, however these studies commonly included multiple experiments and several measures of fitness, resulting in a total of 324 fitness measures for 31 species. For each species we recorded 1) the transmission mode, 2) the type of symbiosis, 3) the mean fitness of the host with and without the symbiont, 4) whether the symbiont was obligate or facultative, 5) environment; whether the experiment included a 'stress' treatment, for example heat stress, presence of a parasitoid or a novel food source.

\section{1) Transmission mode}


We catergorised all symbionts as either having strict vertical transmission, or transmission with at least some horizontal component. We classified species as having strict vertical transmission if they faithfully transmitted symbionts from parent to offspring (Bright \& Bulgheresi 2010). We defined horizontal transmission as acquisition of symbionts from the environment, rather than parents. If a species had predominantly vertical transmission, but potential for transmission to non-offspring, we classified it as having some horizontal transmission. Our classification of transmission mode was therefore conservative with respect to the hypothesis that vertical transmission should lead to higher symbiont cooperation. When the same symbiont was found in multiple hosts, but details about transmission could only be found for one host, we assumed that the transmission mode was the same in all hosts, e.g. Hamiltonella in the pea aphid and black bean aphid.

\section{2) Type of symbiosis}

We classified the type of symbiosis based on the type of role of the symbiont. For example, the Buchnera-aphid symbiosis was classified as 'nutritional', based on evidence from the literature that Buchnera provides aphid hosts with essential amino acids (Baumann 2005). This is in contrast to the Hamiltonella-aphid symbiosis, which we classified as 'defensive' due to evidence that Hamiltonella protects the aphid from parasitism (Guay et al. 2009).

\section{3) Fitness change of host}

We measured host fitness change in two ways, (1) by calculating the percent fitness change and (2) by calculating the effect size of fitness change. 
Percent fitness change: We calculated percent fitness change of the host by calculating the percent change between the mean fitness of the host with the symbiont, and the mean fitness of the host without the symbiont. Experiments included used a variety of methods to cure hosts of their symbionts, including antibiotic treatment and egg sterilisation. The advantage of this measure of fitness change was that only means with and without the symbiont were needed for calculation, and this was particularly useful when studies didn't report variance or sample sizes. For several papers, we calculated the overall mean ourselves or got the data from the supplementary information, e.g. we calculated means from an appendix table given by Castaneda et al. (2010). When only the overall sample size $(\mathrm{N})$ was given, but not the control and treatment sample sizes, we assumed that they were equal (i.e. N/2). We also calculated means and errors directly from plots in published studies.

Effect size of fitness change: An effect size allows comparison, on the same scale, of the results of different studies where a common effect has been measured (Koricheva et al. 2013). For many studies we were unable to calculate an effect size due to papers not reporting variance, statistical tests or sample sizes (in these cases we were only able to calculate percent change in fitness). Where possible, we used the calculation for Hedge's $d$ and Pearson's correlation coefficient $r$ from Boxes 13.2 and 13.3. Where it wasn't possible to directly calculate Pearson's correlation coefficient (due to lack of information about test statistics), we converted from Hedge's $d$ to $r$. We transformed Pearson's $r$ using Fisher's Z transformation $(\mathrm{Zr})$.

We then catergorised the various measures of host fitness used in experiments as (1) fecundity (e.g. number of eggs laid); (2) survival (e.g. longevity); (3) resistance (e.g 
resistance to a pathogen, number of parasite eggs laid); (4) development time (e.g. age at first reproduction); or (5) size/mass based measures of fitness (e.g. body mass or length). By looking at experiments that measure host fitness in different ways, we were able to analyse the data focusing on just host fecundity, rather than all measures of host fitness (including size, mass etc.). Fecundity is likely to be a much more accurate and relevant measure of host fitness, compared to mass or length.

\section{4) Obligate or facultative symbiont}

We defined symbionts as obligate when they are completely host-bound, and have no freeliving stage. We defined symbionts as facultative when they have a free-living stage and are not dependent on the host for reproduction (Oliver et al. 2010).

\section{5) Environment}

We recorded whether symbiont removal was in stressful conditions or unstressful conditions. For example, some experiments removed symbionts and then measured host fitness under stressful (e.g. heat stress, poor nutrient) conditions and also under unstressful conditions (e.g. no stressors, food available).

Our data on mitochondria and chloroplasts comes from the observation that animal and plant cells cannot survive without the presence of these organelles (mitochondria in animals and both in plants). Therefore, if mitochondria or chloroplasts are removed, the fitness change of the host would be $-100 \%$.

\section{Statistical methods}


We examined whether the extent of host-symbiont cooperation was influenced by (1) transmission route (strictly vertical versus some horizontal), (2) the environment (stressed versus unstressed), (3) the type of symbiosis (nutritional or defensive) and (4) whether the symbiont was obligate or facultative. To do this, we used generalized linear mixed models with Markov chain Monte Carlo (MCMC) estimation in R.

We included transmission route, environment, the type of symbiosis and whether the symbiont was obligate or facultative as fixed effects in models. We included a phylogeny of the bacterial symbionts as a random effect to account for non-independence due to shared ancestry. We also included symbiont species and study as random effects to account for non-independence due to species or study conditions. In all models, we used $\%$ fitness change or effect size $Z r$ as the response variable.

The parameter estimates we report are the posterior mode and $95 \%$ credible intervals (lower CI-upper CI), which have been back-transformed to the scale of the response variable (fitness change or effect size $Z r$ ). We calculated estimates of the differences between the levels of fixed effects from a posterior distribution created by subtracting the estimates for each level obtained during each MCMC iteration (labelled 'difference' in Statistical results tables). We considered parameter estimates statistically significant when 95\% credible intervals did not include 0 and pMCMC values calculated in MCMCglmm (number of simulated cases that are $>0$ or less than 0 corrected for finite number of MCMC samples) were less than 0.05 (labelled pdiff in Statistical results tables).

We ran each analysis for 6000000 iterations with a burn-in of 1000000 and thinning interval of 1000. These settings minimised auto-correlation between posterior samples. 
We weighted studies using the inverse of the sample size $(1 / \mathrm{N})$ for analyses with fitness change as the response variable, and we weighted using 1/N-3 for analyses with $Z r$ effect size as the response variable. We checked the convergence of models using GelmanRubin tests in the R package 'coda' (Plummer et al. 2006; Gelman \& Rubin 1992).

To create a phylogeny, we downloaded the nexus file of a recent bacterial phylogeny (Wu et al. 2009) from TreeBase (treebase.org). Where possible, we directly matched species in our database to species used in the Wu et al. (2009) phylogeny, e.g. Burkholderia (in our database) was matched to Burkholderia sp. 383. If a species in our database wasn't included in the Wu et al. (2009) phylogeny, we searched for sister taxa, and where possible found phylogenies including both species for reference. The phylogeny was then trimmed in $\mathrm{R}$ so that it only included the species in our dataset (Fig. 1, Table S2 for details).

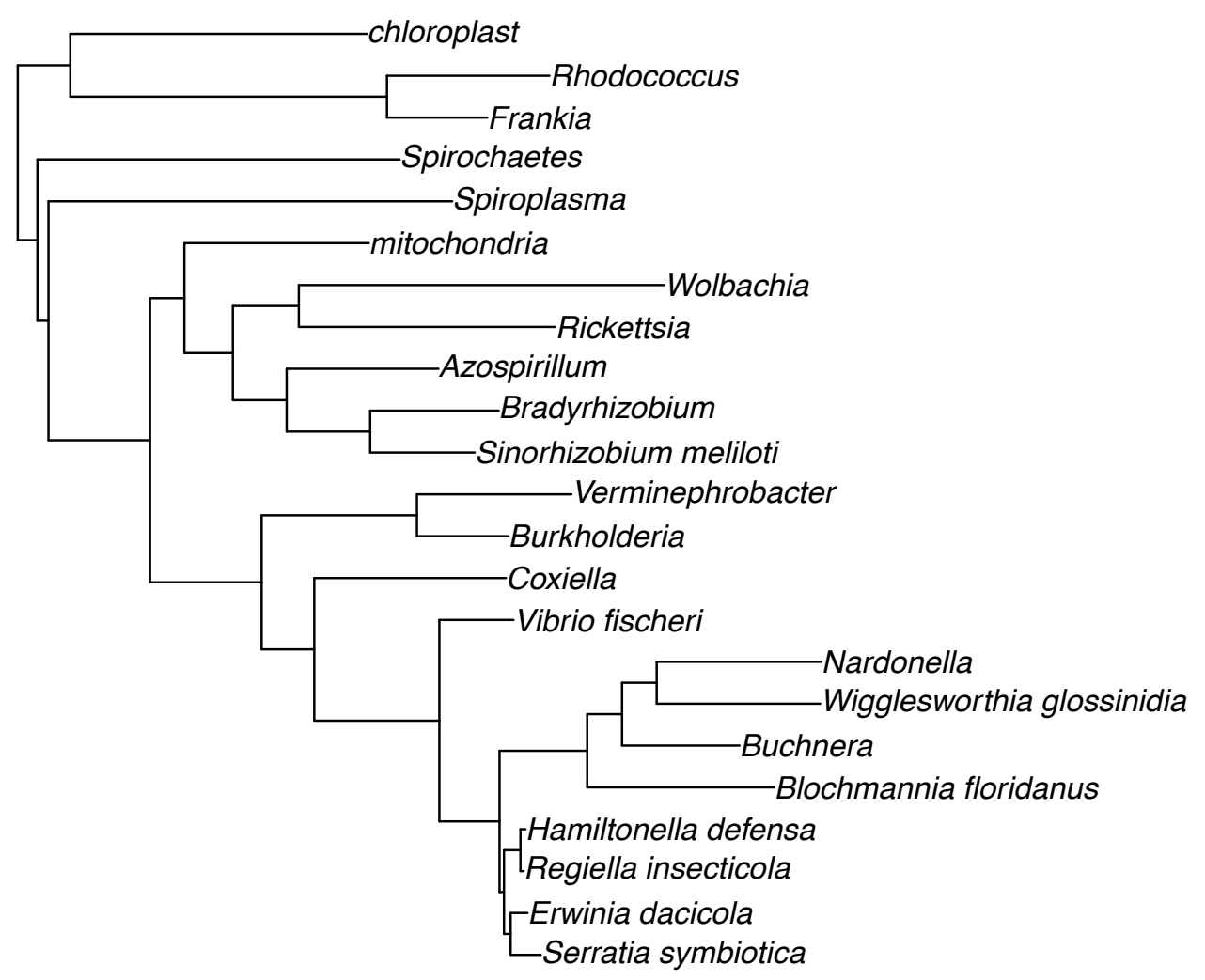


Figure 1: Phylogeny of bacterial symbionts. Constructed from Wu et al. (2009) and edited using Trimtree. See Supplementary Methods for details. 


\section{Results}

\section{The effect of transmission mode and environment on symbiont benefit to host}

The main effects of transmission (strict vertical transmission versus some horizontal transmission) and environment (stressed versus unstressed) are non-significant, but there is a significant interaction between the transmission mode and the environment. We found that horizontally transmitted symbionts were relatively more beneficial in stressed environments compared to unstressed (MCMCglmm, $\mathrm{N}=293$, pMCMC = 0.02, Fig. 2).

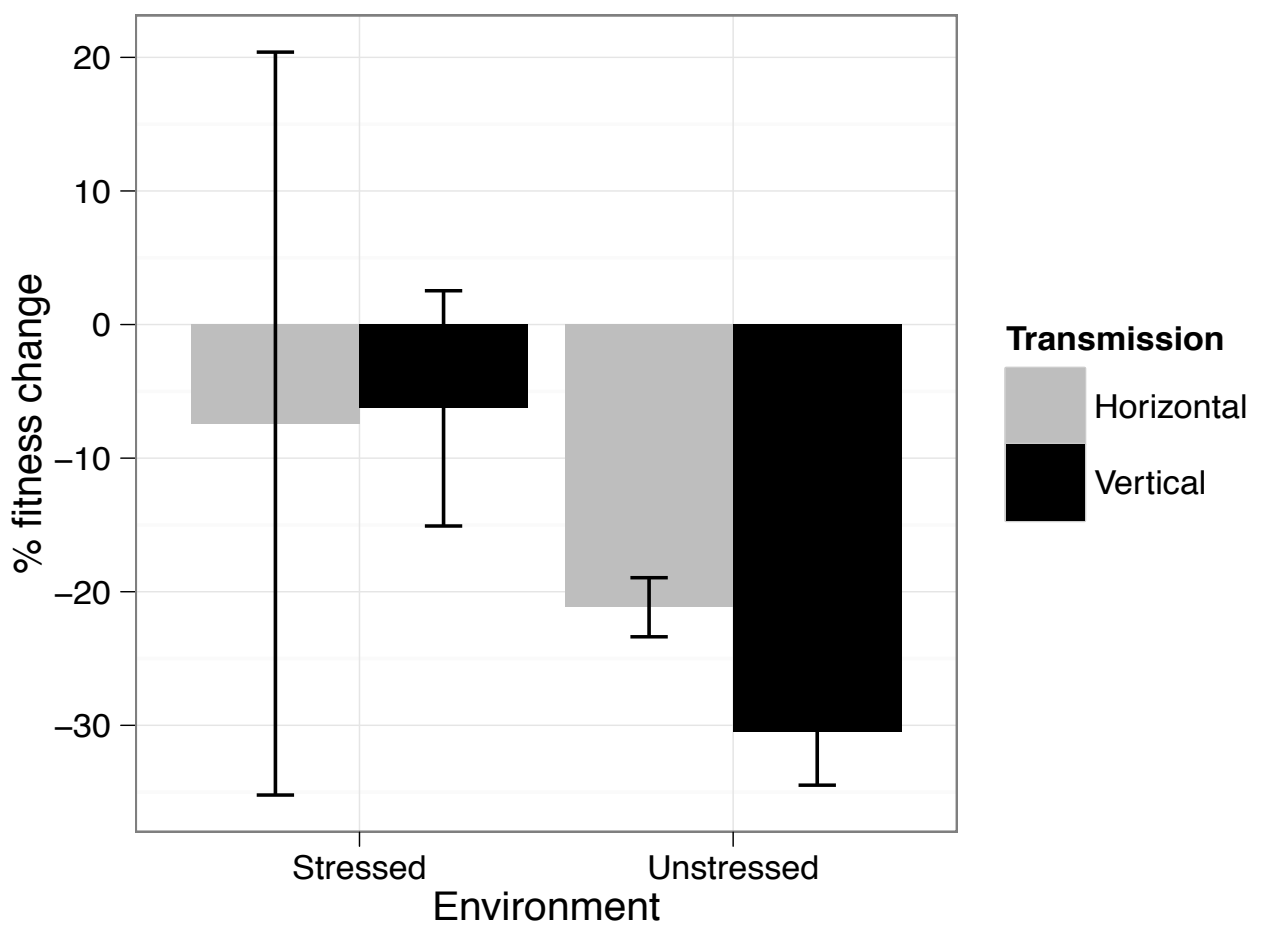

Figure 2: The effect of transmission mode and environment on the benefit of symbionts to their hosts. Horizontally transmitted symbionts were relatively more beneficial in stressed compared to unstressed environments. There was limited data on horizontally transmitted symbionts in stressed environments $(\mathrm{N}=7)$ and the standard error of this estimate is large because of two extreme data points. \% fitness change (y axis) is defined as the percent change in fitness of the host when the symbiont was present, compared to after removal of the symbiont, e.g. if the number of eggs laid dropped from 20 to 10 , this would be a $-50 \%$ change in host fitness. Samples sizes for each treatment are: stressed $\mathrm{N}=49$, unstressed $\mathrm{N}=244$, horizontal $\mathrm{N}=122$, vertical $\mathrm{N}=$ 171. Means and standard error bars are shown; Table S1 for details.

\section{Nutritional and defensive symbioses}


We found that symbionts that provided nutrition to their hosts (e.g. Buchnera) were significantly more beneficial to their hosts than symbionts involved in defence (e.g. Hamiltonella) (MCMCglmm, $\mathrm{N}=170$, pdiff =0.04, Fig. 3).

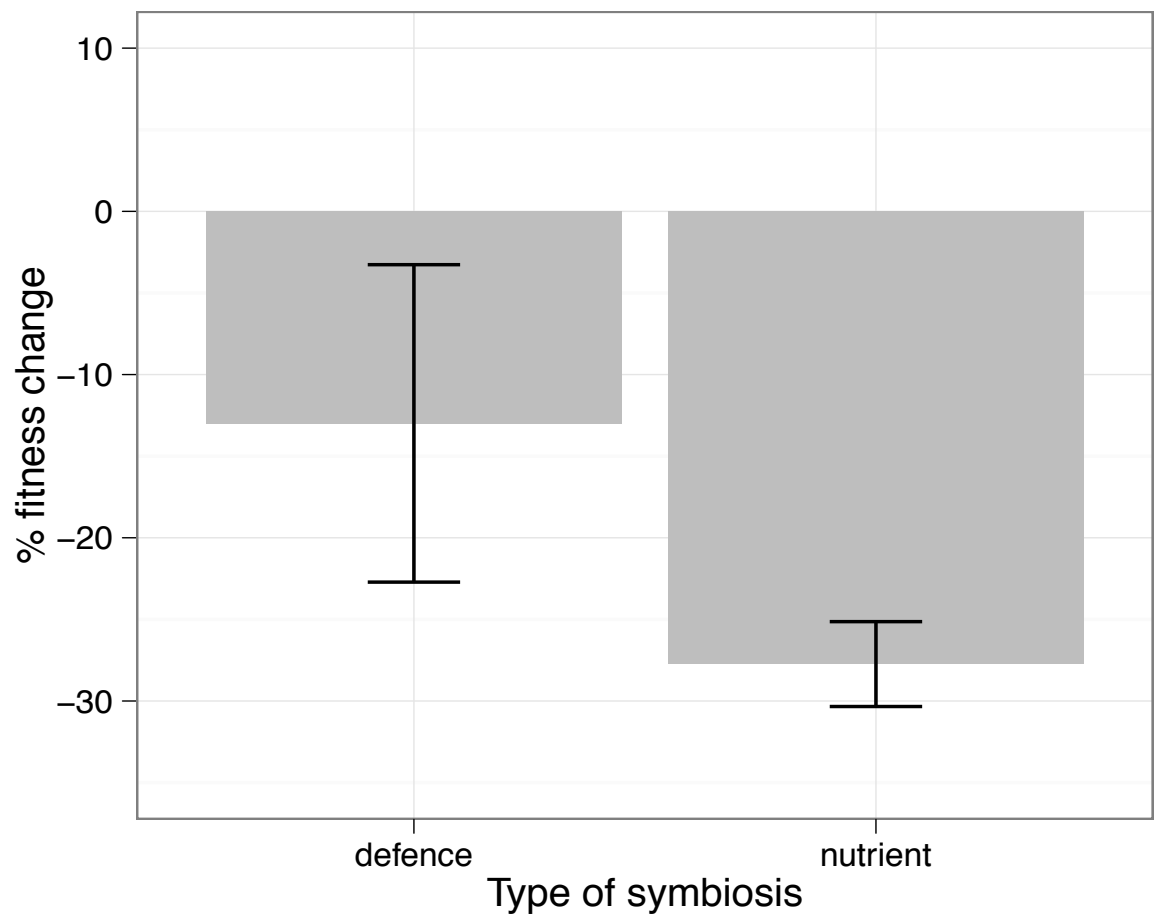

Figure 3: The effect of the type of symbiosis on the benefit of the symbiont to the host. Nutritional symbioses are significantly more beneficial to hosts than defensive symbioses. \% fitness change (y axis) is defined as the percent change in fitness of the host when the symbiont was present, compared to after removal of the symbiont. Sample sizes for each treatment are: defensive $\mathrm{N}=13$ and nutrient $\mathrm{N}=157$. Means and standard errors bars are shown; Table S2 for details.

\section{Obligate and facultative symbioses}

We found that obligate symbionts were not significantly more beneficial to the host that facultative symbionts (MCMCglmm, $\mathrm{N}=292$, pdiff = 0.06, Fig. 4). 


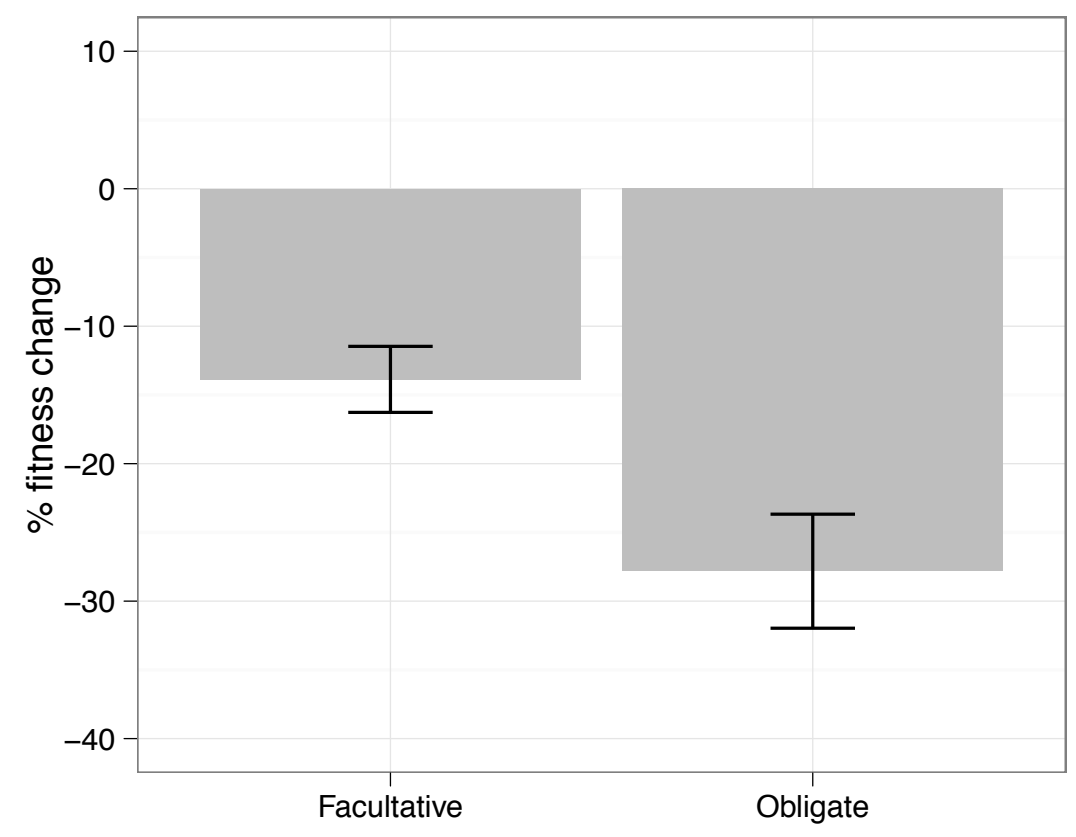

Figure 4: The effect of obligate and facultative symbionts on the benefit to their hosts. $\%$ fitness change (y axis) is defined as the percent change in fitness of the host when the symbiont was present, compared to after removal of the symbiont. There is no significant difference of the benefit to the host between obligate and facultative symbionts (Table S5 for details). Sample sizes are facultative $\mathrm{N}=153$ and obligate $\mathrm{N}=139$. Means and standard error bars are shown.

\section{Robustness of results}

We also investigated whether our results were robust when looking at specific subsets of the data. For example, we tested whether there was a difference in host benefit for vertically versus horizontally transmitted symbionts, only looking at experiments carried out in unstressful conditions and we found no significant difference in host benefit between transmission modes (MCMCglmm, $\mathrm{N}=244$, pdiff $=0.38$, Fig. $5 \mathrm{a}$ ). We ran the same analysis for a further subset of the data, only including experiments that measured host fitness as fecundity (rather than size or mass) and found no significant difference in host benefit between transmission modes (MCMCglmm, $\mathrm{N}=100$, pdiff $=0.67$, Fig. 5b). 
a)

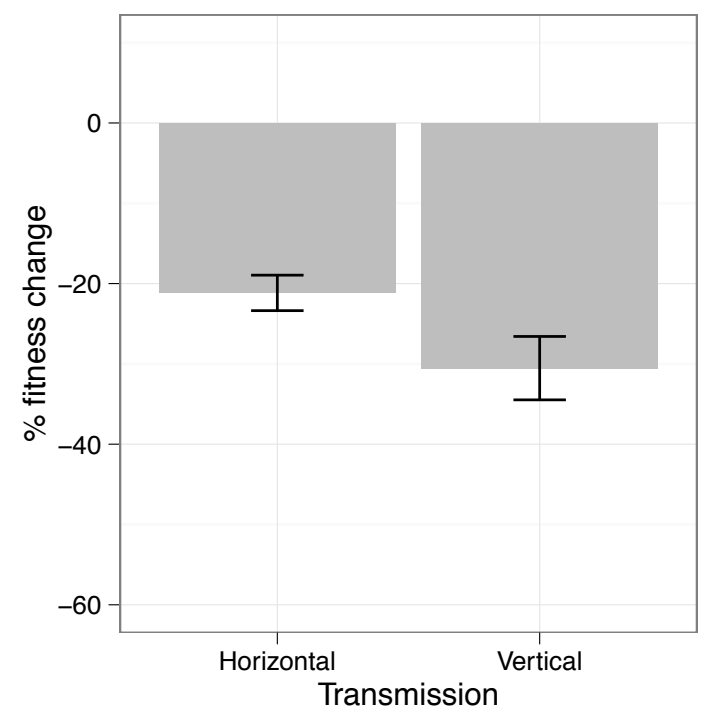

b)

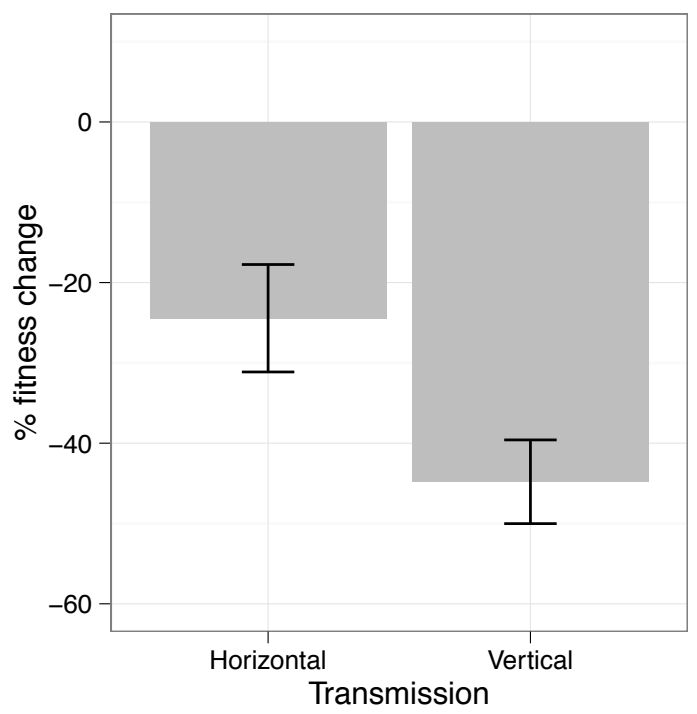

Figure 5: The effect of transmission mode on the benefit of symbionts to their hosts. a) only data from experiments conducted in unstressed environment, including all measures of fitness (horizontal $\mathrm{N}=118$, vertical $\mathrm{N}=126$ ) and $\mathrm{b}$ ) only data from experiments conducted in unstressed environments and with just fecundity as a measure of fitness (horizontal $\mathrm{N}=20$, vertical $\mathrm{N}=80$ ) (Tables S3a and S3b for details). \% fitness change (y axis) is defined as the percent change in fitness of the host when the symbiont was present, compared to after removal of the symbiont. Means and standard error bars are shown.

To confirm that our results were not being influenced by certain species in the dataset (e.g. reproductive manipulators Wolbachia and Rickettsia) we re-ran this analysis having eliminated them. We found that the result remained the same; horizontally transmitted species were relatively more beneficial in stressed versus unstressed environments (MCMCglmm, $\mathrm{N}=203, \mathrm{pMCMC}=0.01$, Table S8). Finally, we ran the analysis without mitochondria and chloroplasts and again, confirmed that our results remained the same (MCMCglmm, $\mathrm{N}=291, \mathrm{pMCMC}=0.01$, Table S6).

\section{The effect of transmission mode and environment: effect size}

We tested whether there was a difference between the effect size $(Z r)$ of host benefit for vertically versus horizontally transmitted symbionts. We found that there was no 
significant difference in benefit to host between transmission modes $(\mathrm{MCMCglmm}, \mathrm{N}=$ 144, pMCMC =0.81, Fig. 6).

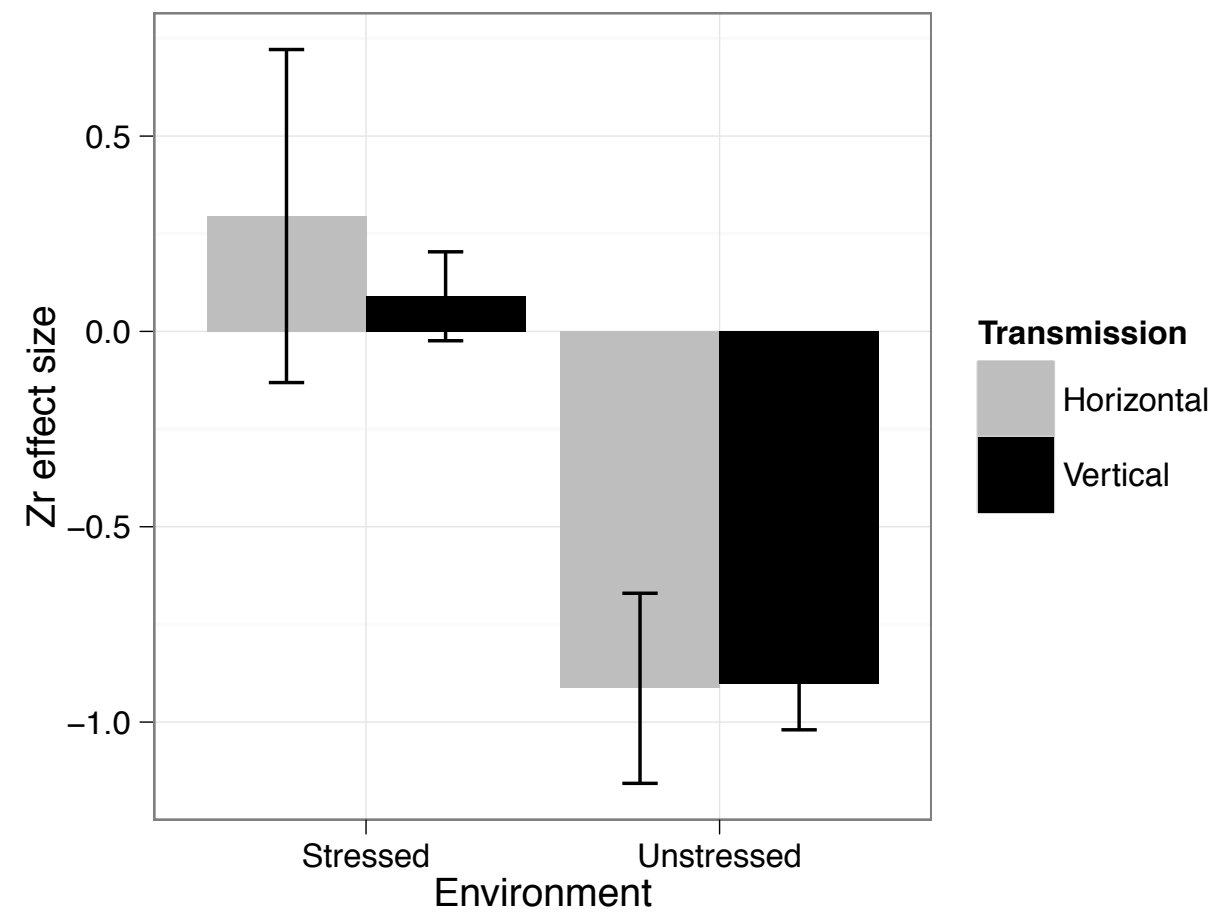

Figure 6: The effect of transmission mode and environment on the benefit of symbionts to their hosts. In this analysis we used $Z r$ (effect size) as opposed to the raw fitness change on symbiont removal. A negative effect size means that the host has a decrease in fitness on symbiont removal, and a positive effect size means the host has an increase in fitness on symbiont removal. An effect size of -1 is equivalent to a $100 \%$ decrease in fitness. The main effects of transmission (strictly vertical transmission versus some horizontal transmission) and environment (stressed versus unstressed) are non-significant, and the interaction between the transmission mode and the environment was also non-significant. Sample sizes for each treatment are: horizontal $\mathrm{N}=23$, vertical $\mathrm{N}=121$, stressed $\mathrm{N}=36$ and unstressed $\mathrm{N}=108$. Means and standard error bars are shown for each effect, Table S4 for details. 


\section{Discussion}

We found that (1) horizontally transmitted symbionts were relatively more beneficial to their hosts in stressful compared to unstressful environments (Fig. 2); (2) nutritional symbionts were more beneficial to hosts than defensive symbionts (Fig. 3); and (3) obligate symbionts were not significantly more beneficial to hosts than facultative ones (Fig. 4). This study demonstrates the importance of considering multiple aspects of symbiont transmission mode and ecology for understanding host-symbiont cooperation.

\section{Transmission mode}

We found no significant difference between vertically and horizontally transmitted symbionts in how much they benefit their hosts (Fig. $2 \& 5$ ). This could be for several reasons. Firstly, this could be due to the conservative way in which we classified transmission mode, where extracellular vertical transmission (e.g. through social contact) was classified as horizontal (because of the potential for transmission to non-offspring). Secondly, it could be because we have included a bacterial phylogeny in the analyses, to control for the effect of shared ancestry, meaning the bacterial symbionts and independent contrasts of transmission mode are clustered in certain areas of the phylogenetic tree.

It is possible that if we included a more phylogenetically diverse set of bacterial symbionts we may see that vertically transmitted symbionts are more beneficial than horizontally transmitted ones. However, this relies on (1) studies on less well-studied symbioses, (2) the ability to experimentally manipulate infection with symbionts and (3) knowledge of transmission mode. Finally, another factor influencing symbiont cooperativeness could be the age of the symbiosis, with older partnerships leading to higher cooperation than 
younger ones (Moran et al. 2008; Dale \& Moran 2006). This is a factor we have not controlled for, but would be a relevant and interesting next step.

\section{Environment}

We found that horizontally transmitted symbionts were relatively more beneficial than vertically transmitted symbionts in stressful environments (i.e. in the presence of a parasitoid, when heat-shocked or when food is limited) (Fig. 2). It has been argued that vertical transmission should lead to the evolution of more cooperative symbionts (Herre et al. 1999) and that horizontal transmission should lead to more diverse and less cooperative symbionts (Douglas 1998a; Frank 1996b). Frank (1996) argued that it is relatedness between symbionts within a host, which is determined by transmission, rather than vertical transmission per se that determines fitness effects on the host.

A wide variety of symbionts are horizontally transmitted between hosts, including all root symbionts (rhizobia, Frankia), Burkholderia, Erwinia and Vibrio fischeri. It has been suggested that horizontal transmission exposes hosts to a wider pool of potential symbiotic partners and so may be useful in unpredictable, novel or stressful environments, where different symbionts would be preferred in different situations (Henry et al. 2013; Oliver et al. 2010; Ebert 2013). Our results support the hypothesis that the benefit of horizontally transmitted symbionts is context dependent and they may be particularly beneficial when the host is under environmental stress.

We focus on transmission mode on an ecological timescale, so even strictly vertically transmitted symbionts could be subject to rare horizontal transmission on an evolutionary/longer timescale. These rare instances of horizontal transmission could result 
in a mixing of different symbiont lineages within a host which would increase conflict between symbionts, potentially having a detrimental effect on host fitness (Frank 1996b; Frank 1996a). If this is the case, our classification would not capture the possibility for within-host conflict brought about by rare events of horizontal transmission.

However, host choice could also be important for determining the degree to which symbionts benefit their host. For example, hosts may use mechanisms such as sanctions (Kiers et al. 2003; Kiers \& Denison 2008) whereby they can eliminate non-cooperative strains, to increase the benefit they receive from symbionts. These mechanisms may be more important in changeable environments, where hosts encounter many different symbionts, and where the benefit of strains may vary (West et al. 2002).

Despite extensive work investigating the benefits of specific symbionts to their hosts (Douglas 1998a; Himler et al. 2011; Chen et al. 2000; Dedeine et al. 2005; Vorburger et al. 2010; Koga et al. 2007; Koga et al. 2003), this is first comparative study looking at how transmission mode affects cooperation across species. There has been analogous experimental work on host-parasite systems demonstrating that vertical transmission of parasites can lead to reduced virulence (Bull et al. 1991; Clayton \& Tompkins 1994; Pagan et al. 2014). Bull et al. (1991) showed that vertically transmitted bacteriophage evolved to be more benevolent towards their host, whereas horizontally transmitted bacteriophage maintained virulence. Clayton and Tompkins (1994) showed that imposing vertical transmission on a parasite of Arabidopsis led to a reduction in harmful effects on the host. This work supports the hypothesis we test here, that vertical transmission leads to higher cooperation between host and symbiont (or host and parasite). 


\section{Types of symbiosis}

We found a significant difference in host benefit between nutritional and defensive symbionts (Fig. 3). This could be for several reasons. Symbionts that provide a nutritional benefit to hosts are likely to be beneficial at all times, whereas symbionts involved in defence are likely to only be beneficial in certain circumstances. This may mean that the selective pressure for cooperation with the host is lower for defensive compared to nutritional symbionts. As well as this, we might expect that high levels of cooperation are harder to attain for symbionts involved in defence against parasitoids, as they are faced with coevolving parasitoid populations that will inevitably vary in time and space. We found relatively few studies that measured the benefit of defensive symbionts in a biologically meaningful way (Russell \& Moran 2006; Łukasik et al. 2013; Brock 2012; Vorburger et al. 2010; Guay et al. 2009), where host benefit was measured in the presence of the relevant parasitoid or stressor, and so it is possible our result is an artefact of the type of data collected rather than a real biological phenomenon. Ultimately, the ideal measure of host fitness would be lifetime reproductive value (Fisher 1958), and most measures included in this analysis were of survival or fecundity, which is probably the best estimate of host fitness that can be measured by these kind of studies. Finally, the exact function of some symbionts is unknown, e.g. Verminephrobacter in earthworms (Lund et al. 2014), so it is also possible that some symbionts have more than one function or that their function varies between hosts.

\section{Obligate versus facultative symbionts}

We found no difference in the benefit to the host of obligate versus facultative symbionts (Fig. 4). This is a somewhat surprising result, as we might predict that obligate symbionts 
(e.g. Buchnera, Serratia, Hamiltonella etc.) that spend their entire life cycle within hosts, would be selected to be cooperative and maximize hosts' reproduction, whereas facultative symbionts (e.g. rhizobia, Vibrio etc.) may not be as cooperative given that they can survive without the host. One possible reason we found no difference in benefit to the host could be that once inside a host, symbionts cannot leave, and so the only way to maximize their fitness is to maximize the fitness of their host, by being cooperative.

This study is limited by the availability of data measuring the fitness effects of symbionts and particularly, the lack of data in understudied groups such as bacterial symbionts of sponges, lichens and corals. However, we have shown that in understanding cooperation between symbionts and their hosts, we need to look at multiple aspects of both symbiont transmission and ecology, and measure the benefit of symbionts in the correct context. 


\section{Supplementary Information}

\section{Statistical Results}

Table S1: Analysis of the effect of transmission and environment on fitness change of the host on symbiont removal, accounting for phylogenetic relationships using MCMCglmm. This analysis included the full dataset.

\begin{tabular}{l|l|l|l|l|l|l}
\hline Effect & & $\mathbf{N}$ & Posterior & Lower CI & Upper CI & p diff \\
\hline Transmission & $\begin{array}{l}\text { Horizontal \& } \\
\text { Vertical }\end{array}$ & 122 & -26.07 & -47.39 & -6.34 & \\
\cline { 2 - 7 } & Ovarial vertical & 171 & -30.92 & -43.53 & -17.67 & \\
\cline { 2 - 7 } & Difference & & 1.58 & -20.35 & 28.81 & $\mathbf{0 . 4 0}$ \\
\hline Phylogeny & & & 1.49 & 0.0002 & 191.29 & \\
\hline Symbiont & & & -0.67 & 0.0002 & 192.78 & \\
\hline Study & & & 804.30 & 444.16 & 1465.01 & \\
\hline Environment & Stressed & 49 & -31.48 & -47.15 & -16.71 & \\
\cline { 2 - 7 } & Unstressed & 244 & -28.31 & -40.87 & -19.14 & \\
\cline { 2 - 7 } & Difference & & -3.24 & -15.85 & 8.95 & $\mathbf{0 . 6 7}$ \\
\hline Phylogeny & & & 2.09 & 0.0002 & 150.42 & \\
\hline Symbiont & & & -0.65 & 0.0002 & 189.67 & \\
\hline Study & & & 796.14 & 427.71 & 1406.40 & \\
\hline Interaction & & & & & & \\
\hline & & & & & & \\
\hline
\end{tabular}

Table S2: Analysis of the effect of the type of symbiosis on fitness change of host on symbiont removal, taking into account phylogenetic relationships using MCMCglmm.

\begin{tabular}{l|l|l|l|l|l|l}
\hline Effect & & N & Posterior & Lower CI & Upper CI & p diff \\
& & & mode & & \\
\hline $\begin{array}{l}\text { Type of } \\
\text { symbiosis }\end{array}$ & Defensive & 13 & -5.67 & -38.90 & 22.95 & \\
\cline { 2 - 7 } & Nutrient & 157 & -38.04 & -53.99 & -25.06 & \\
\cline { 2 - 8 } & Difference & & -28.01 & -64.97 & 4.09 & $\mathbf{0 . 0 4}$ \\
\hline Phylogeny & & & 3.61 & 0.0002 & 349.24 & \\
\hline
\end{tabular}




\begin{tabular}{l|l|l|l|l|l|l}
\hline Symbiont & & & -1.75 & 0.0002 & 441.84 & \\
\hline Study & & & 532.80 & 168.57 & 1225.32 & \\
\hline
\end{tabular}

Table S3a: Analysis of the effect transmission on fitness change of host on symbiont removal, taking into account phylogenetic relationships using MCMCglmm. This analysis was run on a subset of the full dataset eliminating studies that stressed the host.

\begin{tabular}{l|l|l|l|l|l|l}
\hline Effect & & $\mathbf{N}$ & $\begin{array}{l}\text { Posterior } \\
\text { mode }\end{array}$ & Cower & Upper CI & p diff \\
\hline \multirow{2}{*}{ Transmission } & $\begin{array}{l}\text { Horizontal } \\
\text { Vertical }\end{array}$ & 118 & -30.93 & -54.50 & -11.00 & \\
\cline { 2 - 8 } & Ovarial vertical & 126 & -28.91 & -41.17 & -14.54 & \\
\cline { 2 - 8 } & Difference & & -5.61 & -29.71 & 22.66 & $\mathbf{0 . 3 8}$ \\
\hline Phylogeny & & & -0.81 & 0.0003 & 304.79 & \\
\hline Symbiont & & & 2.97 & 0.0002 & 291.30 & \\
\hline Study & & & 1027.77 & 515.34 & 1735.44 & \\
\hline
\end{tabular}

Table S3b: Analysis of the effect transmission on fitness change of host on symbiont removal, taking into account phylogenetic relationships using $\mathrm{MCMCglmm}$. This analysis was run on a subset of the full dataset; 1) eliminating studies that stressed the host and 2) only using fecundity as a measure of fitness.

\begin{tabular}{l|l|l|l|l|l|l}
\hline Effect & & $\mathbf{N}$ & Posterior & Lower & Upper CI & p diff \\
& & mode & CI & & \\
\hline \multirow{2}{*}{ Transmission } & $\begin{array}{l}\text { Horizontal \& } \\
\text { Vertical }\end{array}$ & 20 & -23.62 & -63.14 & 10.22 & \\
\cline { 2 - 7 } & Ovarial vertical & 80 & -35.53 & -48.08 & -18.07 & \\
\cline { 2 - 8 } & Difference & & 7.32 & -28.27 & 49.78 & $\mathbf{0 . 6 7}$ \\
\hline Phylogeny & & & -0.37 & 0.0002 & 152.23 & \\
\hline Symbiont & & & -0.33 & 0.0002 & 155.98 & \\
\hline Study & & & 1207.76 & 719.49 & 2256.52 & \\
\hline
\end{tabular}

Table S4: Analysis of the effect transmission and environment on fitness change (as an effect size, $\mathrm{Zr}$ ) of host on symbiont removal, taking into account phylogenetic relationships using MCMCglmm.

\begin{tabular}{l|l|l|l|l|l|l}
\hline Effect & & $\mathbf{N}$ & Posterior & Lower & Upper CI & $\mathbf{p}$ \\
\hline
\end{tabular}




\begin{tabular}{l|l|l|l|l|l|l}
\hline & & & mode & CI & & diff \\
\hline \multirow{2}{*}{ Transmission } & $\begin{array}{l}\text { Horizontal \& } \\
\text { Vertical }\end{array}$ & 23 & -1.00 & -1.76 & -0.17 & \\
\cline { 2 - 7 } & Ovarial vertical & 121 & -0.74 & -1.08 & -0.28 & \\
\cline { 2 - 7 } & Difference & & -0.30 & -1.08 & 0.71 & $\mathbf{0 . 2 7}$ \\
\hline Phylogeny & & & 0.002 & 0.0001 & 0.20 & \\
\hline Symbiont & & & 0.004 & 0.0002 & 0.17 & \\
\hline Study & & & 0.50 & 0.25 & 1.09 & \\
\hline Environment & Stressed & 36 & -0.70 & -1.09 & -0.25 & \\
\cline { 2 - 8 } & Unstressed & 108 & -0.71 & -1.12 & -0.43 & \\
\cline { 2 - 8 } & Difference & & 0.12 & -0.20 & 0.38 & $\mathbf{0 . 7 3}$ \\
\hline Phylogeny & & & 0.002 & 0.0002 & 0.18 & \\
\hline Symbiont & & & 0.002 & 0.0001 & 0.17 & \\
\hline Study & & & 0.51 & 0.25 & 1.04 & \\
\hline Interaction & & & & & & $\mathbf{0 . 8 1}$ \\
\hline
\end{tabular}

Table S5: Analysis of the effect of obligate versus facultative symbionts on fitness change of host on symbiont removal, taking into account phylogenetic relationships using MCMCglmm.

\begin{tabular}{l|l|l|l|l|l|l}
\hline Effect & & $\mathbf{N}$ & Posterior & Lower & Upper CI & p diff \\
\hline $\begin{array}{l}\text { Obligate or } \\
\text { facultative? }\end{array}$ & Facultative & 153 & -19.17 & -36.19 & -6.90 & \\
\cline { 2 - 7 } & Obligate & 139 & -35.31 & -46.32 & -21.93 & \\
\cline { 2 - 7 } & Difference & & 11.24 & -3.05 & 30.46 & $\mathbf{0 . 0 6}$ \\
\hline Phylogeny & & & -0.29 & 0.0002 & 113.18 & \\
\hline Symbiont & & & -0.25 & 0.0001 & 98.26 & \\
\hline Study & & & 752.57 & 464.02 & 1456.41 & \\
\hline
\end{tabular}

Table S6: Analysis of the effect of transmission and environment on fitness change of the host on symbiont removal, accounting for phylogenetic relationships using MCMCglmm. This analysis was run on a subset of the dataset eliminating mitochondria and chloroplasts.

\begin{tabular}{l|l|l|l|l|l|l}
\hline Effect & & N & Posterior & Lower CI & Upper CI & p diff \\
\hline
\end{tabular}




\begin{tabular}{l|l|l|l|l|l|l}
\hline & & & mode & & & \\
\hline \multirow{2}{*}{ Transmission } & $\begin{array}{l}\text { Horizontal \& } \\
\text { Vertical }\end{array}$ & 122 & -25.02 & -47.58 & -7.82 & \\
\cline { 2 - 7 } & Ovarial vertical & 169 & -25.11 & -40.52 & -15.70 & \\
\cline { 2 - 7 } & Difference & & 5.09 & -22.70 & 23.67 & $\mathbf{0 . 5 2}$ \\
\hline Phylogeny & & & 2.24 & 0.0002 & 216.98 & \\
\hline Symbiont & & & 2.30 & 0.0003 & 189.71 & \\
\hline Study & & & 724.06 & 384.99 & 1348.36 & \\
\hline & Snstressed & 242 & -26.13 & -37.11 & -16.36 & \\
\cline { 2 - 8 } & Difference & & -1.50 & -15.35 & 9.83 & $\mathbf{0 . 3 2}$ \\
\hline Phylogeny & & & 1.41 & 0.0001 & 168.17 & \\
\hline Symbiont & & & -0.62 & 0.0002 & 176.78 & \\
\hline Study & & & 791.50 & 403.14 & 1331.23 & \\
\hline Interaction & & & & & & \\
\hline
\end{tabular}

Table S7: Analysis of the effect of transmission and environment on fitness change of the host on symbiont removal, accounting for phylogenetic relationships using MCMCglmm. This analysis excluded all fecundity data.

\begin{tabular}{l|l|l|l|l|l|l}
\hline Effect & & $\mathbf{N}$ & Posterior & Lower CI & Upper CI & p diff \\
& & & & & \\
\hline \multirow{2}{*}{ Transmission } & $\begin{array}{l}\text { Horizontal \& } \\
\text { Vertical }\end{array}$ & 101 & -23.78 & -39.27 & -8.21 & \\
\cline { 2 - 7 } & Ovarial vertical & 60 & -9.45 & -22.49 & 3.58 & \\
\cline { 2 - 8 } & Difference & & -16.95 & -33.61 & 6.25 & $\mathbf{0 . 0 8}$ \\
\hline Phylogeny & & & -0.21 & 0.0002 & 87.40 & \\
\hline Study & & -0.25 & 0.0003 & 91.52 & \\
\hline Environment & Stressed & 17 & -6.04 & -23.46 & 9.40 & \\
\cline { 2 - 8 } & Unstressed & 144 & -15.38 & -27.13 & -6.04 & \\
\cline { 2 - 7 } & Difference & & 9.80 & -4.80 & 24.79 & $\mathbf{0 . 9 0}$ \\
\hline
\end{tabular}




\begin{tabular}{l|l|l|l|l|l|l}
\hline Phylogeny & & & -0.35 & 0.0003 & 113.61 & \\
\hline Symbiont & & & -0.33 & 0.0001 & 124.33 & \\
\hline Study & & & 410.68 & 151.30 & 921.42 & \\
\hline Interaction & & & & & & $\mathbf{0 . 0 0 5}$ \\
\hline
\end{tabular}

Table S8: Analysis of the effect of transmission and environment on fitness change of the host on symbiont removal, accounting for phylogenetic relationships using MCMCglmm. This analysis excluded reproductive manipulators (Wolbachia, Spiroplasma and Rickettsia).

\begin{tabular}{l|l|l|l|l|l|l}
\hline Effect & & $\mathbf{N}$ & Posterior & Lower CI & Upper CI & p diff \\
& & & mode & & & \\
\hline \multirow{2}{*}{ Transmission } & $\begin{array}{l}\text { Horizontal \& } \\
\text { Vertical }\end{array}$ & 122 & -26.18 & -47.80 & -6.45 & \\
\cline { 2 - 7 } & Ovarial vertical & 81 & -32.26 & -51.59 & -17.69 & \\
\cline { 2 - 8 } & Difference & & 8.97 & -21.28 & 31.79 & $\mathbf{0 . 7 0}$ \\
\hline Phylogeny & & & -0.75 & 0.0002 & 304.66 & \\
\hline Symbiont & & & -0.74 & 0.0002 & 294.88 & \\
\hline Environment & Stressed & 28 & -31.30 & -50.18 & -13.53 & \\
\cline { 2 - 8 } & Unstressed & 175 & -30.29 & -43.82 & -18.32 & \\
\cline { 2 - 8 } & Difference & & -0.02 & -16.05 & 14.92 & $\mathbf{0 . 4 7}$ \\
\hline Phylogeny & & & -0.55 & 0.0002 & 232.33 & \\
\hline Symbiont & & & -0.59 & 0.0002 & 245.44 & \\
\hline Study & & & 749.07 & 279.07 & 1443.93 & \\
\hline Interaction & & & & & & \\
\hline
\end{tabular}




\section{Supplementary Methods}

Table S8: Summary of bacterial symbiosis dataset. Transmission description is given and then the resulting classification of transmission mode that we used in our analyses. If the transmission description allows any potential for horizontal transmission, then we classify the transmission mode as horizontal. The mean fitness change given is the average percent fitness change (on symbiont removal) from all studies and all experiments within each study.

\begin{tabular}{|c|c|c|c|c|c|}
\hline Symbiont & $\begin{array}{l}\text { Transmission } \\
\text { description }\end{array}$ & Classification & $\begin{array}{l}\text { Type of } \\
\text { symbiosis }\end{array}$ & $\begin{array}{l}\text { Mean } \\
\text { fitness } \\
\text { change } \\
(\%) \\
\end{array}$ & Reference(s) \\
\hline Azospirillum & $\begin{array}{l}\text { Environmentally } \\
\text { acquired }\end{array}$ & Horizontal & nutritional & -11.11 & $\begin{array}{l}\text { (Kapulnik et al. } \\
\text { 1981; Kapulnik } \\
\text { et al. 1987) }\end{array}$ \\
\hline $\begin{array}{l}\text { Blochmannia } \\
\text { floridanus }\end{array}$ & $\begin{array}{l}\text { Bacteriocytes, and in } \\
\text { ovaries in females. }\end{array}$ & Vertical & nutritional & -78.53 & $\begin{array}{l}\text { (Feldhaar et al. } \\
\text { 2007) }\end{array}$ \\
\hline Bradyrhizobium & $\begin{array}{l}\text { Environmentally } \\
\text { acquired }\end{array}$ & Horizontal & nutritional & -33.07 & $\begin{array}{l}\text { (Delić \& \& } \\
\text { Stajković- } \\
\text { Srbinović } \\
\text { 2011; Lau et al. } \\
\text { 2012; Hafeez et } \\
\text { al. 2001) }\end{array}$ \\
\hline Buchnera & $\begin{array}{l}\text { Maternally inherited } \\
\text { through ovaries. }\end{array}$ & Vertical & nutritional & -39.19 & $\begin{array}{l}\text { (Douglas 1996; } \\
\text { Caillaud \& } \\
\text { Rahbe 1999) }\end{array}$ \\
\hline Burkholderia & $\begin{array}{l}\text { The symbiont is not } \\
\text { vertically transmitted, } \\
\text { acquired anew every } \\
\text { generation }\end{array}$ & Horizontal & nutritional & -20.28 & $\begin{array}{l}\text { (Kikuchi et al. } \\
\text { 2007) }\end{array}$ \\
\hline $\begin{array}{l}\text { Chemoautotrophic } \\
\text { bacteria of clam }\end{array}$ & $\begin{array}{l}\text { Environmentally } \\
\text { transmitted from a } \\
\text { free-living symbiont } \\
\text { to the new host } \\
\text { generation }\end{array}$ & Horizontal & nutritional & -47.06 & $\begin{array}{lll}\text { (Gros et al. } \\
1998)\end{array}$ \\
\hline Chloroplast & & Vertical & nutritional & -100 & (Keeling 2010) \\
\hline Coxiella & $\begin{array}{l}\text { Coxiella present in } \\
\text { tick eggs- evidence of } \\
\text { transovarial } \\
\text { transmission } \\
\end{array}$ & Vertical & nutritional & -59.75 & $\begin{array}{l}\text { (Zhong et al. } \\
\text { 2007) }\end{array}$ \\
\hline Erwinia & $\begin{array}{l}\text { No evidence for } \\
\text { transovarial } \\
\text { transmission. Each } \\
\text { generation acquires } \\
\text { bacteria anew through } \\
\text { feeding. }\end{array}$ & Horizontal & nutritional & -29.16 & $\begin{array}{l}\text { (de Vries et al. } \\
\text { 2004) }\end{array}$ \\
\hline Erwinia & $\begin{array}{l}\text { The association } \\
\text { extends via the egg } \\
\text { and continues into the } \\
\text { larval stage }\end{array}$ & Vertical & nutritional & -61.69 & $\begin{array}{l}\text { (Ben-Yosef et } \\
\text { al. 2010) }\end{array}$ \\
\hline Frankia & $\begin{array}{l}\text { Horizontal } \\
\text { transmission and free- } \\
\text { living stages }\end{array}$ & Horizontal & nutritional & -61.50 & $\begin{array}{l}\text { (Huss-Danell \& } \\
\text { Frej 1986) }\end{array}$ \\
\hline $\begin{array}{l}\text { Gut microbiota of } \\
\text { bumblebee }\end{array}$ & $\begin{array}{lr}\text { Social } & \text { environment } \\
\text { leads } & \text { to } \\
\text { predominantly vertical }\end{array}$ & Horizontal & defensive & & $\begin{array}{l}\text { Koch \& } \\
\text { Schmid- } \\
\text { Hempel 2011) } \\
\end{array}$ \\
\hline
\end{tabular}




\begin{tabular}{|c|c|c|c|c|c|}
\hline & transmission & & & & \\
\hline Hamiltonella defensa & $\begin{array}{l}\text { Maternally } \\
\text { transmitted, but } \\
\text { secondary (not all } \\
\text { hosts have them). } \\
\text { High fidelity of } \\
\text { vertical transmission. }\end{array}$ & Vertical & defensive & -5.97 & $\begin{array}{l}\text { (Russell \& } \\
\text { Moran 2006; } \\
\text { Oliver et al. } \\
\text { 2006; Guay et } \\
\text { al.2009) }\end{array}$ \\
\hline Mitochondria & & Vertical & nutritional & -100 & $\begin{array}{l}\text { (Cavalier- } \\
\text { Smith 2006) }\end{array}$ \\
\hline Nardonella & $\begin{array}{l}\text { Ovarial transmission- } \\
\text { detected in female } \\
\text { ovaries }\end{array}$ & Vertical & nutritional & -15.68 & $\begin{array}{l}\text { (Kuriwada et } \\
\text { al. 2010) }\end{array}$ \\
\hline Regiella insecticola & $\begin{array}{l}\text { High rates of } \\
\text { transmission from } \\
\text { mother to daugher. }\end{array}$ & Vertical & defensive & -19.24 & $\begin{array}{l}\text { (Leonardo } \\
\text { 2004; Russell } \\
\text { \& Moran 2006; } \\
\text { Vorburger et } \\
\text { al. 2010) }\end{array}$ \\
\hline Rhodococcus rhodnii & uncertain & & nutritional & -7.73 & $\begin{array}{l}\text { (Schaub \& } \\
\text { Eichler 1998) }\end{array}$ \\
\hline Rickettsia & $\begin{array}{l}\text { Maternally } \\
\text { transmitted through } \\
\text { the egg. }\end{array}$ & Vertical & & 8.54 & $\begin{array}{l}\text { (Montllor } \text { et al. } \\
\text { 2002; Chen et } \\
\text { al. } 2000 \text {; } \\
\text { Himler et al. } \\
\text { 2011; Sakurai } \\
\text { et al. 2005; } \\
\text { Chiel et al. } \\
\text { 2009) }\end{array}$ \\
\hline Serratia symbiotica & $\begin{array}{l}\text { No evidence of } \\
\text { horizontal } \\
\text { transmission. }\end{array}$ & Vertical & & -3.32 & $\begin{array}{l}\text { (Chen et al. } \\
\text { 2000; Russell } \\
\text { \& Moran 2006) }\end{array}$ \\
\hline $\begin{array}{l}\text { Sinorhizobium } \\
\text { meliloti }\end{array}$ & $\begin{array}{l}\text { Environmentally } \\
\text { acquired }\end{array}$ & Horizontal & nutritional & -14.26 & $\begin{array}{l}\text { (Heath \& Lau } \\
\text { 2011) }\end{array}$ \\
\hline Spirochaetes bacteria & $\begin{array}{l}\text { Vertically transmitted } \\
\text { but not through } \\
\text { ovaries }\end{array}$ & Horizontal & & -94.73 & $\begin{array}{l}\text { (Eutick et al. } \\
1978)\end{array}$ \\
\hline Spiroplasma & $\begin{array}{ll}\text { Stable } & \text { vertical } \\
\text { transmission } & \text { through } \\
\text { multiple generations. }\end{array}$ & Vertical & & -13.47 & $\begin{array}{l}\text { (Xie et al. } \\
\text { 2010; Xie et al. } \\
\text { 2011; Fukatsu } \\
\text { et al. 2001) }\end{array}$ \\
\hline Verminephrobacter & $\begin{array}{l}\text { Transmitted vertically } \\
\text { via cocoon, where } \\
\text { they are deposited } \\
\text { along with eggs and } \\
\text { sperm }\end{array}$ & Vertical & & -31.05 & $\begin{array}{l}\text { (Lund et al. } \\
\text { 2010; Lund et } \\
\text { al. 2014; } \\
\text { Davidson \& } \\
\text { Stahl 2008) } \\
\end{array}$ \\
\hline $\begin{array}{l}\text { Wigglesworthia } \\
\text { glossinidius }\end{array}$ & $\begin{array}{l}\text { Vertically transmitted } \\
\text { to offspring through } \\
\text { milk glands }\end{array}$ & Vertical & nutritional & -74 & $\begin{array}{lll}\text { Pais } & \text { et } & a l . \\
2008) & & \end{array}$ \\
\hline Wolbachia & $\begin{array}{lr}\text { Maternally } & \\
\text { transmitted } & \text { from } \\
\text { mother to offspring } \\
\text { through } \\
\text { cytoplasm }\end{array}$ & Vertical & nutritional & -40.31 & $\begin{array}{l}\text { (Dedeine et al. } \\
\text { 2005; Fytrou et } \\
\text { al. 2006; Ikeya } \\
\text { et al. 2009) }\end{array}$ \\
\hline
\end{tabular}




\section{Tree creation}

Table S9: Matching species for phylogeny. Species in our database that did not match a species in the Wu et al. (2009) phylogeny directly are included in this table.

\begin{tabular}{|c|c|c|}
\hline $\begin{array}{l}\text { Species in our } \\
\text { dataset }\end{array}$ & Details and reference & $\begin{array}{l}\text { Matched species in Wu et } \\
\text { al. (2009) phylogeny }\end{array}$ \\
\hline Azospirillum & $\begin{array}{l}\text { Magnetospirillum and } \\
\text { Rhodospirillum are included in } \\
\text { the phylogeny, and these are } \\
\text { sister taxa of Azospirilllum }\end{array}$ & $\begin{array}{l}\text { Rhodospirillum_rubrum_at } \\
\text { cc_11170 }\end{array}$ \\
\hline Spirochaetes bacteria & $\begin{array}{l}\text { There are no other spirochaetes } \\
\text { bacteria in our database, so I } \\
\text { entered it as the most basal } \\
\text { spirochaetes bacteria in the Wu } \\
\text { phylogeny }\end{array}$ & $\begin{array}{l}\text { Leptospira_biflexa_serovar } \\
\text { Patoc strain }\end{array}$ \\
\hline $\begin{array}{l}\text { Hamiltonella } \\
\text { defensa }\end{array}$ & $\begin{array}{l}\text { Checked sister taxa relationships } \\
\text { (Degnan et al. 2009), matched } \\
\text { with the closest relative in the } \\
\text { Wu phylogeny }\end{array}$ & Yersinia \\
\hline Nardonella & $\begin{array}{l}\text { Checked sister taxa relationships } \\
\text { (Toju et al. 2013). Matched to a } \\
\text { subspecies of Buchnera to create } \\
\text { the correct branching } \\
\text { relationships between Buchnera, } \\
\text { Nardonella and Wigglesworthia }\end{array}$ & $\begin{array}{l}\text { Buchnera_aphidicola_str } \\
\text { Cc_cinara }\end{array}$ \\
\hline Mitochondria & $\begin{array}{l}\text { Ancestral bacterial species is } \\
\text { known to be an } \\
\text { alphaproteobacteria (Cavalier- } \\
\text { Smith 2006), so entered as most } \\
\text { basal alphaproteobacteria in Wu } \\
\text { phylogeny }\end{array}$ & Magnetococcus sp. \\
\hline Chloroplast & $\begin{array}{l}\text { Ancestral bacterial species } \\
\text { known to be a cyanobacteria } \\
\text { (Keeling 2010), so entered as } \\
\text { most basal cyanobacteria on Wu } \\
\text { phylogeny. }\end{array}$ & Gloeobacter_violaceus \\
\hline
\end{tabular}




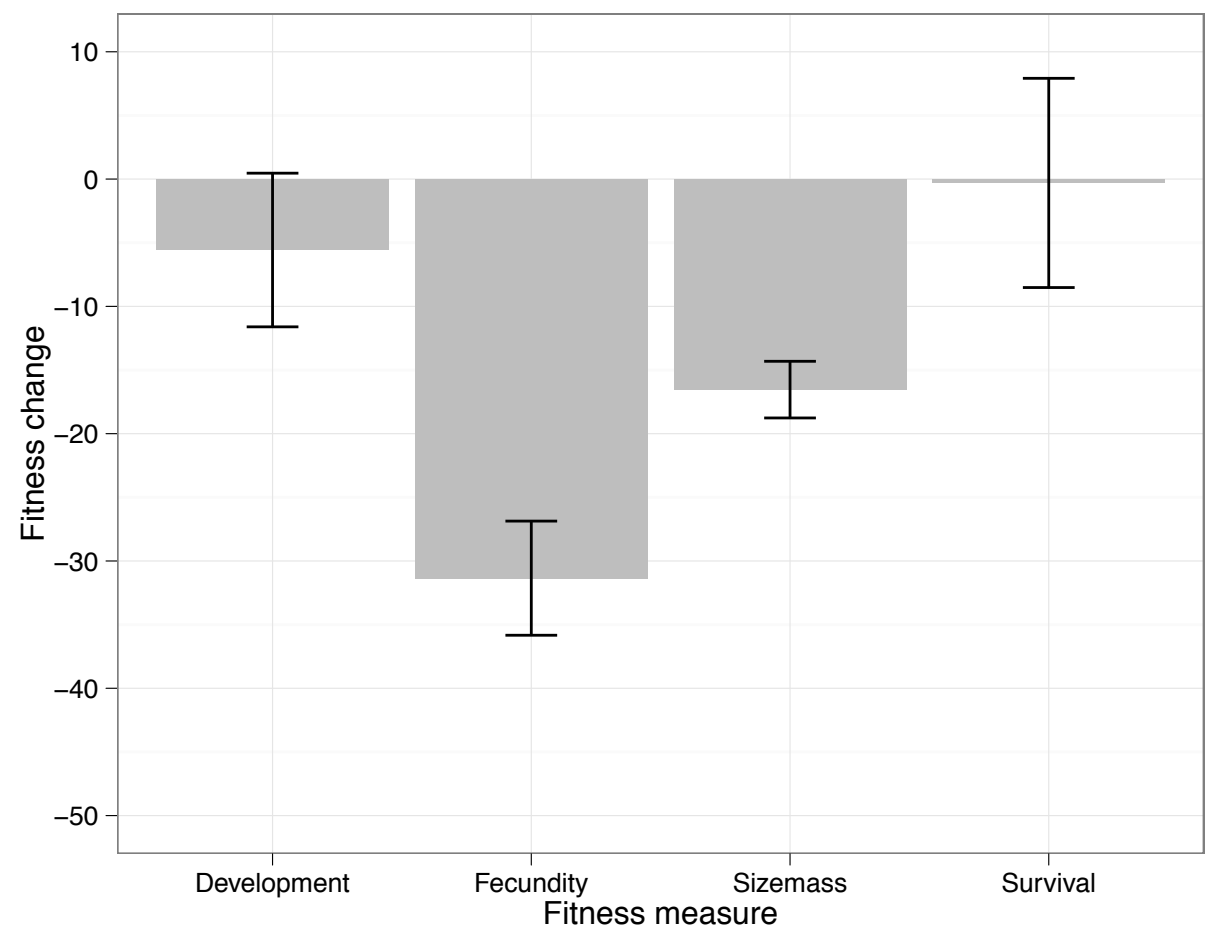

Figure S1: Host fitness change for different measures of fitness. Means and standard errors are shown.

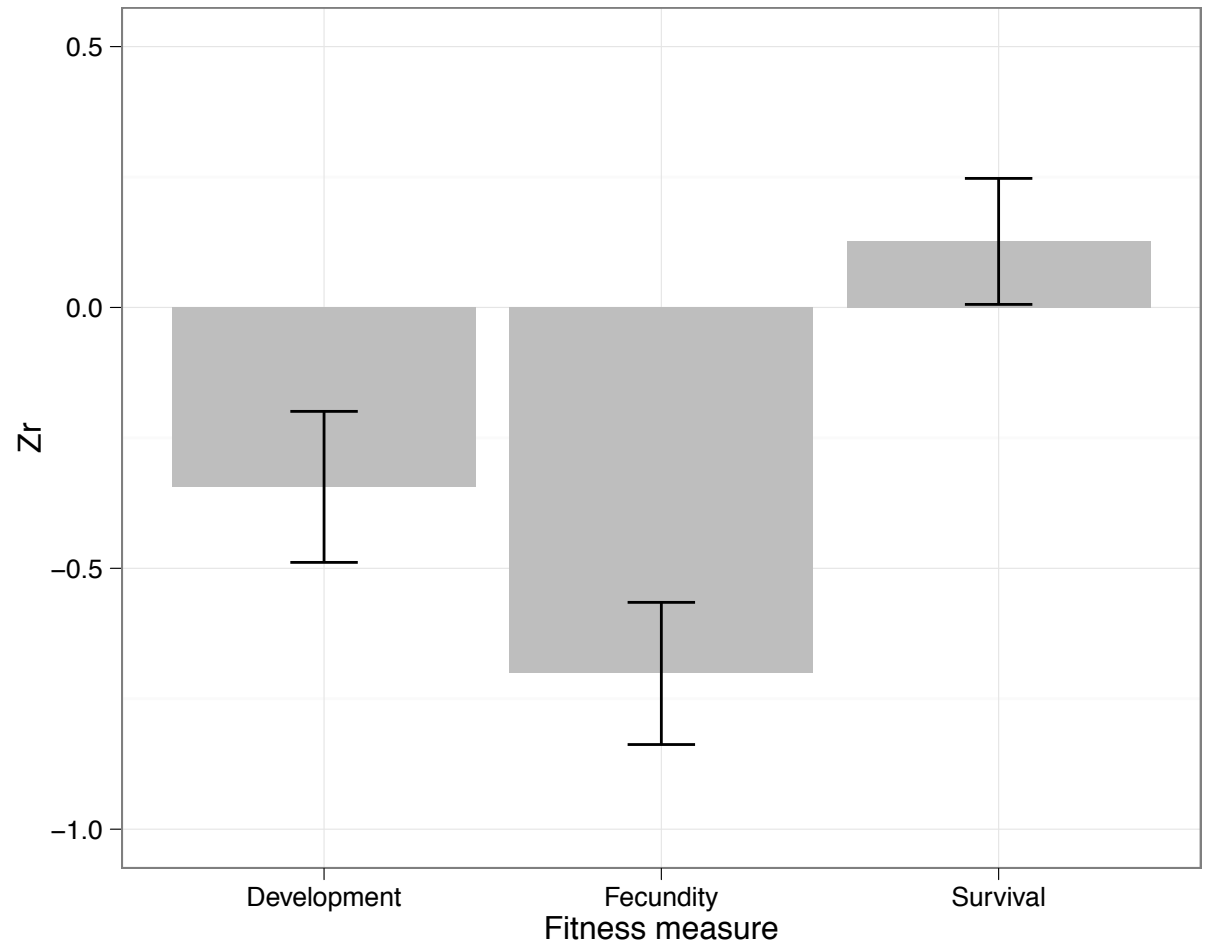

Figure S2: Effect size (Zr) of host fitness change for different measures of fitness. Means and standard errors are shown. 


\section{Chapter 5: Discussion}

Each chapter contains an extensive discussion section relevant to that particular study, so here I only discuss the general results and themes of this thesis. The aim of this thesis has been to identify common principles that apply to both within- and between- species major transitions. I have focused on how conflict between individuals is minimised in social groups, potentially leading to the evolution of cooperation, complexity and, if conditions allow, a major evolutionary transition in individuality.

\section{Within-species transitions}

In Chapters $2 \& 3$ I focused on the within-species transition to multicellularity. In Chapter 2, I used the evolution of multicellularity as a model system for understanding how the way social groups form influences relatedness between individuals, and thus the evolution of complexity and the likelihood of a major transition. Multicellularity has evolved many times on the tree of life in diverse groups of organisms, but I found that the major transition to obligate multicellularity has only occurred when multicellular organisms form through clonal group formation (where cells stick together after division). These results are analogous to and support comparative work on another within-species transition, the evolution of eusociality (Boomsma 2007, 2009), showing that obligate eusociality has only evolved through subsociality and strict lifetime monogamy (Hughes et al. 2008).

There is potential to extend the comparative work in Chapter 2 in several ways. The dataset could be used to investigate the types of environments that may favour clonal or non-clonal group formation. Bonner (1998) suggested that aquatic environments should favour clonal group formation and terrestrial environments may favour non-clonal group 
formation, due to the physical constraints of forming a group in aquatic environments (Bonner 1998). It would also be possible to investigate whether the benefit of multicellularity, e.g. dispersal in Dictyostelium or defence against predation in algae, correlates with the complexity of multicellularity and whether or not a major transition has taken place.

In Chapter 3, I investigated how predation pressure can promote the formation of multicellular groups in $C$. vulgaris. This suggests that predation pressure could be important in understanding the evolution of multicellularity. Studying the initial stages of social group formation is important for understanding major evolutionary transitions, because the route of group formation determines within-group conflict through its influence on genetic relatedness between individuals (Queller 2000; Bourke 2011). I would like to extend this experimental work to investigate how multicellular groups form in C. vulgaris, and test whether this varies depending on predation pressure. There is also potential to test comparatively whether the route of group formation in colony-forming green algae influences complexity and cooperation, given that there are examples of clonal and non-clonal group formation within the algae (Table 1).

Table 1: Routes to social group formation, induced by predation, in a diverse range of algal species. Clonal group formation is when cells stick together after division, and non-clonal group formation is when cells aggregate together to form groups. This list is not intended to be exhaustive.

\begin{tabular}{|c|c|c|c|}
\hline Algal species & Predator & $\begin{array}{l}\text { Proposed route to } \\
\text { social group } \\
\text { formation }\end{array}$ & Reference \\
\hline Chlorella vulgaris & Ochromonas vallescia & Clonal & (Boraas et al. 1998) \\
\hline Cyanobium sp. & Ochromonas sp. & Non-clonal & $\begin{array}{l}\text { (Jezberová \& Komárková } \\
\text { 2007) }\end{array}$ \\
\hline $\begin{array}{l}\text { Microcystis } \\
\text { aeruginosa }\end{array}$ & $\begin{array}{l}\text { Ochromonas sp. and } \\
\text { other zooplankton }\end{array}$ & Non-clonal & $\begin{array}{l}\text { (Yang et al. 2009; Yang et } \\
\text { al. 2005) }\end{array}$ \\
\hline $\begin{array}{l}\text { Scenedesmus } \\
\text { subspicatus }\end{array}$ & Daphnia magna & Clonal & $\begin{array}{lrrr}\text { (Lurling et al. } & 1997 ; \\
\text { Hessen \& Van } & \text { Donk } \\
1993) & & & \\
\end{array}$ \\
\hline
\end{tabular}




\begin{tabular}{llll}
\hline $\begin{array}{l}\text { Scenedesmus } \\
\text { acutus }\end{array}$ & Daphnia magna & Clonal & (Lurling et al. 1997) \\
$\begin{array}{l}\text { Scenedesmus } \\
\text { dimorphus }\end{array}$ & Daphnia magna & Clonal & (Ha et al. 2004; Lurling et \\
& & al. 1997) \\
\hline
\end{tabular}

\section{Between-species transitions}

In Chapter 4, I focused on the between-species transition to symbiosis. I investigated how transmission route and environment influence how beneficial symbionts are to hosts. Although transmission mode has been predicted to determine how cooperative symbionts should be (through its influence on within-host symbiont relatedness), I found that this effect depended on environmental conditions. This study highlights the importance of considering transmission, the type of benefit symbionts confer to their hosts and environmental factors in understanding the evolution of symbiosis. This study could be extended to include fungi, which would then add other ecologically important symbioses such as leaf cutter ants, mycorrhizae and lichens.

In this thesis I have used both within- and between-species transitions to investigate how social groups form and what factors lead to negligible conflict between individuals, in order to understand when and why major transitions in evolution occur. This work highlights (1) the power of using broad scale comparative studies to answer questions about major transitions, (2) the potential of using facultatively multicellular algae as a model system to understand the initial stages of a transition and (3) the two conditions, namely complete mutual dependence and negligible conflict, needed for a major transition in individuality are the same for within- and between-species transitions. 


\section{References}

Aanen, D.K., de Fine Licht, H.H., Debets, A.J.M, Kerstes, N.A.G., Hoekstra, R.F., Boomsma, J.J., 2009, High symbiont relatedness staibilizes mutualistic cooperation in fungus-growing termites, Science, 326, 1103-1106.

Baumann, P., 2005. Biology bacteriocyte-associated endosymbionts of plant sap-sucking insects. Anпu Rev Microbiol, 59, 155-189.

Becks, L., Ellner, S.P., Jones, L.E., Hairston Jr, N.G., 2010. Reduction of adaptive genetic diversity radically alters eco-evolutionary community dynamics. Ecology Letters, 13, 989997.

Ben-Yosef, M., Aharon, Y., Jurkevitch, E., Yuval, B., 2010. Give us the tools and we will do the job: symbiotic bacteria affect olive fly fitness in a diet-dependent fashion. Proceedings of the Royal Society B: Biological Sciences, 277, 1545-1552.

Bonner, J.T., 1998. The origins of multicellularity. Integrative Biology Issues News and Reviews, $1,27-36$.

Boomsma, J.J., 2007. Kin selection versus sexual selection: why the ends do not meet. Current Biology, 17, 673-683.

Boomsma, J.J., 2009. Lifetime monogamy and the evolution of eusociality. Philosophical Transactions of the Royal Society B: Biological Sciences, 364, 3191-3207.

Boomsma, J.J., 2013. Beyond promiscuity: mate-choice commitments in social breeding. Philosophical Transactions of the Royal Society B: Biological Sciences, 368, 20120050.

Boraas, M., Seale, D. \& Boxhorn, J., 1998. Phagotrophy by a flagellate selects for colonial prey: a possible origin of multicellularity. Evolutionary Ecology, 12, 153-164.

Bourke, A.F.G., 2011. Principles of Social Evolution, Oxford Series in Ecology and Evolution.

Bourke, A.F.G., 2014. Hamilton's rule and the causes of social evolution. Philosophical Transactions of the Royal Society B: Biological Sciences, 369, 20130362.

Bright, M. \& Bulgheresi, S., 2010. A complex journey: transmission of microbial symbionts. Nature Reviews Microbiology, 8, 218-230.

Brock, D., 2012. Evolutionary costs and benefits of a newly discovered symbiosis between the social amoeba Dictyostelium and bacteria. Doctoral Thesis: Rice University.

Bull, J.J., Molineux, I.J. \& Rice, W.R., 1991. Selection of benevolence in a host-parasite system. Evolution, 45, 875-882.

Burki, F. \& Pawlowski, J., 2006. Monophyly of Rhizaria and multigene phylogeny of unicellular bikonts. Molecular biology and evolution, 23, 1922-1930.

Caillaud, C.M. \& Rahbe, Y., 1999. Aposymbiosis in a cereal aphid: reproductive failure and influence on plant utilization. Ecological Entomology, 24, 111-114.

Cavalier-Smith, T., 2006. Origin of mitochondria by intracellular enslavement of a photosynthetic purple bacterium. Proceedings of the Royal Society B: Biological Sciences, 273, 1943-1952. 
Chen, D.-Q., Montllor, C.B. \& Purcell, A.H., 2000. Fitness effects of two facultative endosymbiotic bacteria on the pea aphid, Acyrthosiphon pisum, and the blue alfalfa aphid, $A$. kondoi. Entomologia Experimentalis et Applicata, 95, 315-323.

Chiel, E., Inbar, M., Mozes-Daube, N., White, J.A., Hunter, M.S., Zchori-Fein, E., 2009. Assessments of fitness effects by the facultative symbiont Rickettsia in the sweetpotato whitefly (Hemiptera: Aleyrodidae). Ecology and Population Biology, 102, 413-418.

Claes, M.F. \& Dunlap, P.V., 2000. Aposymbiotic culture of the sepiolid squid Euprymna scolopes: role of the symbiotic bacterium Vibrio fischeri in host animal growth, development, and light organ morphogenesis. The Journal of experimental zoology, 286, 280-296.

Claessen, D., Rozen, D.E., Kuipers, O.P., Sogaard-Andersen, L., van Wezel, G.P., 2014. Bacterial solutions to multicellularity: a tale of biofilms, filaments and fruiting bodies. Nature Reviews Microbiology, 12, 115-124.

Clayton, D.H. \& Tompkins, D.M., 1994. Ectoparasite virulence is linked to mode of transmission. Proceedings of the Royal Society B: Biological Sciences, 256, 211-217.

Corno, G. \& Jurgens, K., 2006. Direct and indirect effects of protist predation on population size structure of a bacterial strain with high phenotypic plasticity. Applied and Environmental Microbiology, 72, 78-86.

Cornwallis, C.K., West, S.A., Davis, K.E., Griffin, A.S., 2010. Promiscuity and the evolutionary transition to complex societies. Nature, 466, 969-972.

Crespi, B.J. \& Yanega, D., 1995. The definition of eusociality. Behavioral Ecology, 6, 109-155.

Davidson, S.K. \& Stahl, D.A., 2008. Selective recruitment of bacteria during embryogenesis of an earthworm. The ISME journal, 2, 510-518.

Davies, N.B., Krebs, J.R. \& West, S.A, 2012. An Introduction to Behavioural Ecology 4 ed., Wiley-Blackwell.

de Vries, E.J., Jacobs, G., Sabelis, M.W., Menken, S.B.J., Breeuwer, J.A.J., 2004. Diet-dependent effects of gut bacteria on their insect host: the symbiosis of Erwinia sp. and western flower thrips. Proceedings of the Royal Society B: Biological Sciences, 271, 2171-2178.

Dedeine, F., Boulétreau, M. \& Vavre, F., 2005. Wolbachia requirement for oogenesis: occurrence within the genus Asobara (Hymenoptera, Braconidae) and evidence for intraspecific variation in A. tabida. Heredity, 95, 394-400.

Degnan, P.H., Yu, Y., Sisneros, N., Wing, R.A., Moran, N.A., DeLong, E.F., 2009. Hamiltonella defensa, genome evolution of protective bacterial endosymbiont from pathogenic ancestors. Proceedings of the National Academy of Sciences of the United States of America, 106, 90639068 .

Delić, D. \& Stajković-Srbinović, O., 2011. Effect of bradyrhizobial inoculation on growth and seed yield of mungbean in fluvisol and humofluvisol. African Journal of Microbiology, 5, 3946-3957.

Diggle, S.P., Griffin, A.S., Campbell, G.C., West, S.A., 2007. Cooperation and conflict in quorum-sensing bacterial populations. Nature, 450, 411-414.

Douglas, A.E., 1998a. Host benefit and the evolution of specialization in symbiosis. Heredity, 81, 
599-603.

Douglas, A.E., 1998b. Nutritional interactions in insect-microbial symbioses: aphids and their symbiotic bacteria Buchnera. Annual Review of Entomology, 43, 17-37.

Douglas, A.E., 1996. Reproductive failure and the free amino acid pools in pea aphids (Acyrthosiphon pisum) lacking symbiotic bacteria. Journal of Insect Physiology, 42, 247-255.

Ebert, D., 2013. The epidemiology and evolution of symbionts with mixed-mode transmission. Annual Review of Ecology, Evolution, and Systematics, 44, 623-643.

Eutick, M.L., Veivers, P., O'Brien, R.W., Slaytor, M., 1978. Dependence of the higher termite, Nasutitermes exitiosus and the lower termite, Coptotermes lacteus on their gut flora. Journal of Insect Physiology, 24, 363-368.

Feldhaar, H., Straka, J., Krischke, M., Berthold, K., Stoll, S., Mueller, M.J., Gross, R., 2007. Nutritional upgrading for omnivorous carpenter ants by the endosymbiont Blochmannia. BMC Biology, 5.

Felsenstein, J., 2012. A comparative method for both discrete and continuous characters using the threshold model. The American Naturalist, 179, 145-156.

Fisher, R.A., 1958. The genetical theory of natural selection, Oxford University Press.

Fisher, R.M., Cornwallis, C.K. \& West, S.A, 2013. Group formation, relatedness, and the evolution of multicellularity. Current Biology, 23, 1120-1125.

Frank, S.A., 1996a. Host-symbiont conflict over the mixing of symbiotic lineages. Proceedings of the Royal Society B: Biological Sciences, 263, 339-344.

Frank, S.A., 1996b. Models of parasite virulence. The Quarterly Review of Biology, 71, 37-78.

Frank, S.A., 1995. Mutual policing and repression of competition in the evolution of cooperative groups. Nature, 377, 520-522.

Freshwater, D.W. \& Rueness, J., 1994. Phylogenetic relationships of some European Gelidium (Gelidiales, Rhodophyta) species, based on rbcL nucleotide sequence analysis. Phycologia, 33, 187-194.

Fukatsu, T., Tsuchida, T., Nikoh, N., Koga, R., 2001. Spiroplasma symbiont of the pea aphid, Acyrthosiphon pisum (Insecta: Homoptera). Applied and Environmental Microbiology 67, 1284-1291

Fytrou, A., Schofield, P.G., Kraaijeveld, A.R., Hubbard, S.F., 2006. Wolbachia infection suppresses both host defence and parasitoid counter-defence. Proceedings of the Royal Society B: Biological Sciences, 273, 791-796.

Gardner, A. \& Grafen, A., 2009. Capturing the superorganism: a formal theory of group adaptation. Journal of Evolutionary Biology, 22, 659-671.

Gavrilets, S., 2010. Rapid transition towards the division of labor via evolution of developmental plasticity. PLoS computational biology, 6, e1000805.

Gelman, A. \& Rubin, D.B., 1992. Inference from iterative simulation using multiple sequences. Statistical science, 7, 457-472. 
Gilbert, O.M., Foster, K.R, Mehdiabadi, N.J., Strassmann, J.E, Queller, D.C, 2007. High relatedness maintains multicellular cooperation in a social amoeba by controlling cheater mutants. Proceedings of the National Academy of Sciences of the United States of America, 104, 8913-8917.

Griffin, A.S., West, S. \& Buckling, A., 2004. Cooperation and competition in pathogenic bacteria. Nature, 430, 1024-1027.

Gros, O., Frenkiel, L. \& Moueza, M., 1998. Gill filament differentiation and experimental colonization by symbiotic bacteria in aposymbiotic juveniles of Codakia orbicularis (Bivalvia: Lucinidae). Invertebrate Reproduction \& Development, 34, 219-231.

Grosberg, R.K. \& Strathmann, R.R, 1998. One cell, two cell, red cell, blue cell: the persistence of a unicellular stage in multicellular life histories. Trends in Ecology \& Evolution, 13, 112-116.

Grosberg, R.K. \& Strathmann, R.R., 2007. The evolution of multicellularity: A minor major transition? Annual Review of Ecology Evolution and Systematics, 38, 621-654.

Guay, J-F., Boudreault, S., Michaud, D., Cloutier, C., 2009. Impact of environmental stress on aphid clonal resistance to parasitoids: role of Hamiltonella defensa bacterial symbiosis in association with a new facultative symbiont of the pea aphid. Journal of Insect Physiology, 55, 919-926.

Ha, K., Jang, M. \& Takamura, N., 2004. Colony formation in planktonic algae induced by zooplankton culture media filtrate. Journal of Freshwater Ecology, 19, 9-16.

Hadfield, J., 2010. MCMC methods for multi-response generalized linear mixed models: the MCMCglmm R package. Journal of Statistical Software, 33, 1-22.

Hadfield, J.D. \& Nakagawa, S., 2010. General quantitative genetic methods for comparative biology: phylogenies, taxonomies and multi-trait models for continuous and categorical characters. Journal of Evolutionary Biology, 23, 494-508.

Hafeez, F., Hameed, S., Ahmad, T., Malik, K., 2001. Competition between effective and less effective strains of Bradyrhizobium spp. for nodulation on Vigna radiata. Biology and Fertility of Soils, 33, 382-386.

Hahn, M.W., Moore, E. \& Höfle, M.G., 1999. Bacterial filament formation, a defense mechanism against flagellate grazing, is growth rate controlled in bacteria of different phyla. Applied and Environmental Microbiology, 65, 25-35.

Hallmann, A., 2011. Evolution of reproductive development in the volvocine algae. Sexual plant reproduction, 24, 97-112.

Hamilton, W.D., 1964. The genetical evolution of social behaviour. I \& II. Journal of theoretical biology, 7, 1-52.

Hammerschmidt, K., Rose, C.J., Kerr, B., Rainey, P.B., 2014, Life cycles, fitness decoupling and the evolution of multicellularity. Nature, 515, 75-79.

Harmon, L.J., Weir, J.T., Brock, C.D., Glor, R.E., Challenger, W., 2008. Geiger: investigating evolutionary radiations. Bioinformatics, 24, 129-131.

Harvey, P.H., \& Purvis, A., 1991. Comparitive methods for explaining adaptations. Nature, 351, 619-624. 
Hatchwell, B.J., 2009. The evolution of cooperative breeding in birds: kinship, dispersal and life history. Philosophical Transactions of the Royal Society B: Biological Sciences, 364, $3217-$ 3227.

Heath, K.D. \& Lau, J.A., 2011. Herbivores alter the fitness benefits of a plant-rhizobium mutualism. Acta Oecologica, 37, 87-92.

Heath-Heckman, E.A.C., Peyer, S.M., Whistler, C.A., Apicella, M.A., Goldman, W.E., McFallNgai, M.J., 2013. Bacterial bioluminescence regulates expression of a host cryptochrome gene in the squid-Vibrio symbiosis. mBio, 4, e00167-13.

Henry, L.M., Peccoud, J., Simon, J-C., Hadfield, J.D., Maiden, M.J.C., Ferrari, J., Godfray, C.J., 2013. Horizontally transmitted symbionts and host colonization of ecological niches. Current Biology, 23, 1713-1717.

Herre, E.A., Knowlton, N., Mueller, U.G., Rehner, S.A., 1999. The evolution of mutualisms: exploring the paths between conflict and cooperation. Trends in Ecology \& Evolution, 14, 4953.

Hessen, D.O. \& Van Donk, E., 1993. Morphological changes in Scenedesmus induced by substances released from Daphnia. Archiv Fur Hydrobiologie, 127, 129-140.

Himler, A.G., Adachi-Hagimori, T., Bergen, J.E., Kozuch, A., Kelly, S.E., Tabashnik, E., Chiel, E., Duckworth, V.E., Dennehy, T.J., Zchori-Fein, E., Hunter, M.S., 2011. Rapid spread of a bacterial symbiont in an invasive whitefly is driven by fitness benefits and female bias. Science, 332, 254-256.

Hosokawa, T., Koga, R., Kikuchi, Y., Meng, X-Y., Fukatsu, T., 2010. Wolbachia as a bacteriocyte-associated nutritional mutualist. Proceedings of the National Academy of Sciences of the United States of America, 107, 769-774.

Hughes, W.O.H., Oldroyd, B.P., Beekman, M., Ratnieks, F.L.W., 2008. Ancestral monogamy shows kin selection is key to the evolution of eusociality. Science, 320, 1213-1216.

Huss-Danell, K. \& Frej, A.-K., 1986. Distribution of Frankia in soils from forest and afforestation sites in northern Sweden. Plant and Soil, 90, 407-417.

Ikeya, T., Broughton, S., Alic, N., Grandison, R., Partridge, L., 2009. The endosymbiont Wolbachia increases insulin/IGF-like signalling in Drosophila. Proceedings of the Royal Society B: Biological Sciences, 276, 3799-3807.

Jakobsen, H.H. \& Tang, K.W., 2002. Effects of protozoan grazing on colony formation in Phaeocystis globosa (Prymnesiophyceae) and the potential costs and benefits. Aquatic Microbial Ecology, 27, 261-273.

James, T.Y., Kauff, F., Schoch, C.L., Matheny, P.B., Hofstetter, V., Cox, C.J. et al., 2006. Reconstructing the early evolution of Fungi using a six-gene phylogeny. Nature, 443, 818 822.

Jezberová, J. \& Komárková, J., 2007. Morphological transformation in a freshwater Cyanobium $s p$. induced by grazers. Environmental microbiology, 9, 1858-1862.

Kaltenpoth, M., 2009. Actinobacteria as mutualists: general healthcare for insects? Trends in Microbiology, 17, 529-535. 
Kapulnik, Y., Sarig, S., Nur, I., Okon, Y., 1981. Yield increases in summer cereal crops in Israeli fields inoculated with Azospirillum. Experimental Agriculture, 17, 179-187.

Kapulnik, Y., Okon, Y. \& Henis, Y., 1987. Yield response of spring wheat cultivars (Triticum aestivum and T. turgidum) to inoculation with Azospirillum brasilense under field conditions. Biology and Fertility of Soils, 4, 27-35.

Keeling, P.J., 2010. The endosymbiotic origin, diversification and fate of plastids. Philosophical Transactions of the Royal Society B: Biological Sciences, 365, 729-748.

Keller, L., 1999. Levels of selection in evolution. Princeton University Press.

Kiers, E.T. \& Denison, R.F., 2008. Sanctions, cooperation, and the stability of plant-rhizosphere mutualisms. Annual review of ecology, 39, 215-236.

Kiers, E.T., Rousseau, R.A., West, S.A., Denison, R.F., 2003. Host sanctions and the legumerhizobium mutualism. Nature, $425,78-81$.

Kikuchi, Y., Hosokawa, T. \& Fukatsu, T., 2007. Insect-microbe mutualism without vertical transmission: a stinkbug acquires a beneficial gut symbiont from the environment every generation. Applied and Environmental Microbiology, 73, 4308-4316.

Koch, H. \& Schmid-Hempel, P., 2011. Socially transmitted gut microbiota protect bumble bees against an intestinal parasite. Proceedings of the National Academy of Sciences of the United States of America, 108, 19288-19292.

Koga, R., Tsuchida, T., Sakurai, M., Fukatsu, T., 2007. Selective elimination of aphid endosymbionts: effects of antibiotic dose and host genotype, and fitness consequences. Fems Microbiology Ecology, 60, 229-239.

Koga, R., Tsuchida, T. \& Fukatsu, T., 2003. Changing partners in an obligate symbiosis: a facultative endosymbiont can compensate for loss of the essential endosymbiont Buchnera in an aphid. Proceedings of the Royal Society B: Biological Sciences, 270, 2543-2550.

Korb, J., Buschmann, M., Schafberg, S., Liebig, J., Bagneres, A.G., 2012. Brood care and social evolution in termites. Proceedings of the Royal Society B: Biological Sciences, 279, 26622671 .

Koricheva, J., Gurevitch, J. \& Mengersen, K., 2013. Handbook of Meta-analysis in Ecology and Evolution, Princeton University Press.

Kuriwada, T., Hosokawa, T., Kumano, N., Shirimoto, K., 2010. Biological role of Nardonella endosymbiont in its weevil host. PLOS ONE, 5, 1-7.

Kuzdzal-Fick, J.J., Fox, S.A., Strassmann, J.E., Queller, D.C., 2011. High relatedness is necessary and sufficient to maintain multicellularity in Dictyostelium. Science, 334, 1548-1551.

Lampert, W., Rothhaupt, K.O. \& Elert, von, E., 1994. Chemical induction of colony formation in a green alga (Scenedesmus acutus) by grazers (Daphnia). Limnology and Oceanography, 39, $1543-1550$.

Lau, J.A., Bowling, E.J., Gentry, L.E., Glasser, P.A., Monarch, E.A., Olesen, W.M., Waxmonsky, J., Young, R.T., 2012. Direct and interactive effects of light and nutrients on the legumerhizobia mutualism. Acta Oecologica, 39, 80-86.

Leigh, E., Jr, 1995. The major transitions of evolution. Evolution, 49, 1302-1306. 
Leonardo, T.E., 2004. Removal of a specialization-associated symbiont does not affect aphid fitness. Ecology Letters, 7, 461-468.

Long, J.D., Smalley, G.W., Barsby, T., Anderson, J.T., Hay, M.E., 2007. Chemical cues induce consumer-specific defenses in a bloom-forming marine phytoplankton. Proceedings of the National Academy of Sciences of the United States of America, 104, 10512-10517.

Lukas, D. \& Clutton-Brock, T., 2012. Cooperative breeding and monogamy in mammalian societies. Proceedings of the Royal Society B: Biological Sciences, 279, 2151-2156.

Łukasik, P., Dawid, M.A., Ferrari, J., Godfray, H.C.J., 2013. The diversity and fitness effects of infection with facultative endosymbionts in the grain aphid, Sitobion avenae. Oecologia, 173, 985-996.

Lund, M.B., Holmstrup, M., Lomstein, B.A., Damgaard, C., Schramm, A., 2010. Beneficial effect of Verminephrobacter nephridial symbionts on the fitness of the earthworm Aporrectodea tuberculata. Applied and Environmental Microbiology, 76, 4738-4743.

Lund, M.B., Kjeldsen, K.U. \& Schramm, A., 2014. The earthworm - Verminephrobacter symbiosis: an emerging experimental system to study extracellular symbiosis. Frontiers in Microbiology, 5.

Lurling, M. \& Van Donk, E., 2000. Grazer-induced colony formation in Scenedesmus: are there costs to being colonial? Oikos, 88, 111-118.

Lurling, M. \& Van Donk, E., 1997. Morphological changes in Scenedesmus induced by infochemicals released in situ from zooplankton grazers. Limnology and Oceanography, 42, $783-788$.

Lurling, M. \& Van Donk, E., 1996. Zooplankton-induced unicell-colony transformation in Scenedesmus acutus and its effect on growth of herbivore Daphnia. Oecologia, 108, 432-437.

Lurling, M., De Lange, H.J. \& Van Donk, E., 1997. Changes in food quality of the green alga Scenedesmus induced by Daphnia infochemicals: biochemical composition and morphology. Freshwater Biology, 38, 619-628.

MacLean, R.C. \& Gudelj, I., 2006. Resource competition and social conflict in experimental populations of yeast. Nature, $441,498-501$.

Mayeli, S.M., Nandini, S. \& Sarma, S.S.S., 2005. The efficacy of Scenedesmus morphology as a defense mechanism against grazing by selected species of rotifers and cladocerans. Aquatic Ecology, 38, 515-524.

Maynard Smith, J. \& Szathmáry, E., 1995. The Major Transitions in Evolution, Oxford University Press.

Mayr, E., 1961. Cause and effect in biology. Science, 134, 1501-1506.

Michod, R., 2007. Evolution of individuality during the transition from unicellular to multicellular life. Proceedings of the National Academy of Sciences of the United States of America, 104, 8613-8618.

Michod, R.E., 1997. Cooperation and conflict in the evolution of individuality: multilevel selection of the organism. American Naturalist, 149, 607-645.

Michod, R.E. \& Roze, D., 2001. Cooperation and conflict in the evolution of multicellularity. 
Heredity, 86, 1-7.

Mieog, J.C., Olsen, J.L., Berkelmans, R., Bleuler-Martinez, S.A., Willis, B.L., van Oppen, M.J.H., 2009. The roles and interactions of symbiont, host and environment in defining coral fitness. PLOS ONE, 4, e6364.

Montllor, C.B., Maxmen, A. \& Purcell, A.H., 2002. Facultative bacterial endosymbionts benefit pea aphids Acyrthosiphon pisum under heat stress. Ecological Entomology, 27, 189-195.

Moran, N.A., McCutcheon, J.P. \& Nakabachi, A., 2008. Genomics and evolution of heritable bacterial symbionts. Annual Review of Genetics, 42, 165-190.

Nakagawa, S. \& Freckleton, R.P., 2010. Model averaging, missing data and multiple imputation: a case study for behavioural ecology. Behavioral Ecology and Sociobiology, 65, 103-116.

Oliver, K.M., Russel, J.A., Moran, N.A., Hunter, M.S., 2003. Facultative bacterial symbionts in aphids confer resistance to parasitic wasps. Proceedings of the National Academy of Sciences of the United States of America, 100, 1803-1807.

Oliver, K.M., Degnan, P.H., Burke, G.R., Moran, N.A., 2010. Facultative symbionts in aphids and the horizontal transfer of ecologically important traits. Annual Review of Entomology, 55, $247-266$.

Oliver, K.M., Moran, N.A. \& Hunter, M.S., 2006. Costs and benefits of a superinfection of facultative symbionts in aphids. Proceedings of the Royal Society B: Biological Sciences, 273, $1273-1280$.

Pagan, I., Montes, N., Milgroom, M.G., Garcia-Arenal, F., 2014. Vertical transmission selects for reduced virulence in a plant virus and for increased resistance in the host. Plos Pathogens, 10.

Pagel, M., 1994. Detecting correlated evolution on phylogenies: a general method for the comparative analysis of discrete characters. Proceedings of the Royal Society B: Biological Sciences, 255, 37-45.

Pagel, M., 1999. Inferring the historical patterns of biological evolution. Nature, 401, 877-884.

Pais, R., Lohs, C., Wu, Y., Wang, J., Aksoy, S., 2008. The obligate mutualist Wigglesworthia glossinidia influences reproduction, digestion, and immunity processes of its host, the tsetse fly. Applied and Environmental Microbiology, 74, 5965-5974.

Paps, J., Medina-Chacon, L.A., Marshall, W., Suga, H., Ruiz-Trillo, I., 2012. Molecular phylogeny of unikonts: new insights into the position of Apusomonads and Ancyromonads and the internal relationships of Opisthokonts. Protist, 164, 2-12.

Paradis, E., Claude, J. \& Strimmer, K., 2004. APE: Analyses of phylogenetics and evolution in R language. Bioinformatics, 20, 289-290.

Pike, N., Whitfield, J.A. \& Foster, W.A., 2007. Ecological correlates of sociality in Pemphigus aphids, with a partial phylogeny of the genus. BMC Evolutionary Biology, 7, 185.

Plummer, M., Best, N., Cowles, K., Vines, K., 2006. Coda: convergence diagnosis and output analysis for MCMC. $R$ news, 6, 1-25.

Purvis, A., Gittleman, J.L. \& Luh, H.-K., 1994. Truth or consequences: effects of phylogenetic accuracy on two comparative methods. Journal of theoretical biology, 167, 293-300. 
Queller, D.C., 2000. Relatedness and the fraternal major transitions. Philosophical Transactions of the Royal Society B: Biological Sciences, 355, 1647-1655.

Queller, D.C. \& Strassmann, J.E., 2009. Beyond society: the evolution of organismality. Philosophical Transactions of the Royal Society B: Biological Sciences, 364, 3143-3155.

Ratcliff, W.C., Denison, R.F., Borrello, M., Travisano, M., 2012, Experimental evolution of multicellularity. Proceedings of the National Academy of Sciences of the United States of America, 109, 1595-1600.

Ratcliff, W.C., Herron, M.D., Howell, K., Pentz, J.T., Rosenzweig, F., Travisano, M., 2013, Experimental evolution of an alternating uni- and multicellular life cycle in Chlamydomonas reinhardtii, Nature Communications, 4.

Revell, L.J., 2011. phytools: an R package for phylogenetic comparative biology (and other things). Methods in Ecology and Evolution, 3, 217-223.

Rossetti, V., Schirrmeister, B.E., Bernasconi, M.V., Bagheri, H.C., 2010. The evolutionary path to terminal differentiation and division of labor in cyanobacteria. Journal of theoretical biology, $262,23-34$.

Russell, J.A. \& Moran, N.A., 2006. Costs and benefits of symbiont infection in aphids: variation among symbionts and across temperatures. Proceedings of the Royal Society B: Biological Sciences, 273, 603-610.

Sachs, J., Ehinger, M.O. \& Simms, E.L., 2010. Origins of cheating and loss of symbiosis in wild Bradyrhizobium. Journal of Evolutionary Biology, 23, 1075-1089.

Sachs, J.L. \& Wilcox, T.P., 2006. A shift to parasitism in the jellyfish symbiont Symbiodinium microadriaticum. Proceedings of the Royal Society B: Biological Sciences, 273, 425-429.

Sachs, J.L., Skophammer, R.G. \& Regus, J.U., 2011. Evolutionary transitions in bacterial symbiosis. Proceedings of the National Academy of Sciences of the United States of America, 108, 10800-10807.

Sakurai, M., Koga, R., Tsuchida, T., Meng, X-Y., Fukatsu, T., 2005. Rickettsia symbiont in the pea aphid Acyrthosiphon pisum: novel cellular tropism, effect on host fitness, and interaction with the essential symbiont Buchnera. Applied and Environmental Microbiology 71, 40694075 .

Schaub, G.A. \& Eichler, S., 1998. The effects of aposymbiosis and of an infection with Blastocrithidia triatomae (Trypanosomatidae) on the tracheal system of the reduviid bugs Rhodnius prolixus and Triatoma infestans. Journal of Insect Physiology, 44, 131-140.

Schirrmeister, B.E., Antonelli, A. \& Bagheri, H.C., 2011. The origin of multicellularity in cyanobacteria. BMC Evolutionary Biology, 11, 45.

Shultz, S., Opie, C. \& Atkinson, Q.D., 2011. Stepwise evolution of stable sociality in primates. Nature, 479, 219-222.

Silberfeld, T., Leigh, J.W., Verbruggen, H., Cruaud, C., de Reviers, B., Rousseau, F., 2010. A multi-locus time-calibrated phylogeny of the brown algae (Heterokonta, Ochrophyta, Phaeophyceae): investigating the evolutionary nature of the "brown algal crown radiation". Molecular phylogenetics and evolution, 56, 659-674. 
Stanley, S., 1973. An ecological theory for the sudden origin of multicellular life in the late Precambrian, Proceedings of the National Academy of Sciences of the United States of America, 70, 1486-1489.

Strassmann, J.E. \& Queller, D.C., 2010. The social organism: congresses, parties, and committees. Evolution, 64, 605-616.

Tinbergen, N., 1963. On aims and methods of ethology. Zeitschrift für Tierpsychologie, 20, 410433.

Toju, H., Tanabe, A.S., Notsu, Y., Sota, T., Fukatsu, T., 2013. Diversification of endosymbiosis: replacements, co-speciation and promiscuity of bacteriocyte symbionts in weevils. The ISME journal, 7, 1378-1390.

Van Donk, E., Ianora, A. \& Vos, M., 2011. Induced defences in marine and freshwater phytoplankton: a review. Hydrobiologia, 668, 3-19.

Vorburger, C., Gehrer, L. \& Rodriguez, P., 2010. A strain of the bacterial symbiont Regiella insecticola protects aphids against parasitoids. Biology Letters, 6, 109-111.

West, S.A., Kiers, E.T., Simms, E.L., Denison, R.F., 2002. Sanctions and mutualism stability: why do rhizobia fix nitrogen? Proceedings of the Royal Society B: Biological Sciences, 269, 685694.

West, S., Griffin, A.S. \& Gardner, A., 2007. Evolutionary explanations for cooperation. Current Biology, 17, 1-12.

Williams, T.A., Foster, P.G., Nye, T.M.W., Cox, C.J., Embley, T.M., 2012. A congruent phylogenomic signal places eukaryotes within the Archaea. Proceedings of the Royal Society B: Biological Sciences, 279, 4870-4879.

Wu, D., Hugenholtz, P., Mavromatis, K., Pukall, R., Dalin, E., Ivanova, N.N. et al., 2009. A phylogeny-driven genomic encyclopaedia of Bacteria and Archaea. Nature, 462, 1056-1060.

Xie, J., Tiner, B., Vilchez, I., Mateos, M., 2011. Effect of the Drosophila endosymbiont Spiroplasma on parasitoid wasp development and on the reproductive fitness of waspattacked fly survivors. Evolutionary Ecology, 25, 1065-1079.

Xie, J., Vilchez, I. \& Mateos, M., 2010. Spiroplasma bacteria enhance survival of Drosophila hydei attacked by the parasitic wasp Leptopilina heterotoma. PLoS ONE, 5, 1-7.

Yamamura, N., 1993. Vertical transmission and evolution of mutualism from parasitism. Theoretical Population Biology, 44, 95-109.

Yang, Z. \& Li, J.J., 2007. Effects of Daphnia-associated infochemicals on the morphology and growth of Scenedesmus obliquus and Microcystis aeruginosa. Journal of Freshwater Ecology, $22,249-253$.

Yang, Z., Kong ,F., Zhang, M., Yu, Y., Qian, S., 2009. Benefits and costs of the grazer-induced colony formation in Microcystis aeruginosa. International Journal of Limnology, 45, 203208.

Yang, Z., Kong, F., Shi, X, Cao, H., 2006. Morphological response of Microcystis aeruginosa to grazing by different sorts of zooplankton. Hydrobiologia, 563, 225-230.

Yang, Z., Kong, F. \& Shi, X., 2005. Effects of filtered lake water on colony formation and growth 
rate in Microcystis aeruginosa of different physiological phases. Journal of Freshwater Ecology, 20, 425-429.

Zhong, J., Jasinskas, A. \& Barbour, A.G., 2007. Antibiotic treatment of the tick vector Amblyomma americanum reduced reproductive fitness. PLoS ONE, 2, 1-7. 
Appendix 1: Group formation, relatedness and the evolution of multicellularity 


\section{Group Formation, Relatedness, and the Evolution of Multicellularity}

\author{
Roberta M. Fisher, ${ }^{1}$ Charlie K. Cornwallis, ${ }^{2}$ \\ and Stuart A. West ${ }^{1, *}$ \\ ${ }^{1}$ Department of Zoology, University of Oxford, Oxford OX1 \\ 3PS, UK \\ ${ }^{2}$ Department of Biology, Lund University, Lund 223 62, \\ Sweden
}

\section{Summary}

The evolution of multicellular organisms represents one of approximately eight major evolutionary transitions that have occurred on earth [1-4]. The major challenge raised by this transition is to explain why single cells should join together and become mutually dependent, in a way that leads to a more complex multicellular life form that can only replicate as a whole. It has been argued that a high genetic relatedness $(r)$ between cells played a pivotal role in the evolutionary transition from single-celled to multicellular organisms, because it leads to reduced conflict and an alignment of interests between cells [1-17]. We tested this hypothesis with a comparative study, comparing the form of multicellularity in species where groups are clonal $(r=1)$ to species where groups are potentially nonclonal $(r \leq 1)$. We found that species with clonal group formation were more likely to have undergone the major evolutionary transition to obligate multicellularity and had more cell types, a higher likelihood of sterile cells, and a trend toward higher numbers of cells in a group. More generally, our results unify the role of group formation and genetic relatedness across multiple evolutionary transitions and provide an unmistakable footprint of how natural selection has shaped the evolution of life [1].

\section{Results and Discussion}

The evolution of life on earth, from the simplest replicating molecules to complex animal societies, has involved approximately eight major evolutionary transitions in individuality $[2-4,18]$. In each of these transitions, a group of individuals that could previously replicate independently joins together to form a new, more complex life form that can only replicate as a whole. For example, genes come together to form genomes, cells join together to form multicellular organisms, and multicellular organisms join together to form eusocial societies. The major challenge raised by each of these transitions is to explain why individuals should join together and become mutually dependent in a way that leads to a more complex individual $[2-4,18]$.

Here, we use a phylogenetically based [19] comparative study to test how the relatedness between interacting cells influenced both the likelihood of the major evolutionary transition to obligate multicellularity and the level of sociality in multicellular groups. We obtained data on 168 species, with representatives from all multicellular lineages except diatoms and charophyte algae (Figure 1; Figure S1 available online). We used life-history data on how groups form to infer relatedness.

*Correspondence: stuart.west@zoo.ox.ac.uk
Specifically, when groups form by cells remaining with their parents, then groups are clonal ( $r=1 ; 149$ species). This usually involves the group going through a single-celled (unicellular) stage $[10,11]$. In contrast, if groups form by cells aggregating together, then relatedness could be anything from zero to one $(r \leq 1)$ but is likely to be nonclonal $(r<1 ; 19$ species) [20].

Transitions to Obligate Multicellularity

We first examined whether relatedness influenced the likelihood that groups underwent the transition to obligate multicellularity. Our distinction here is between obligately multicellular species, which can only complete their life cycle as a multicellular organism, and facultatively multicellular species, which are able to complete their life cycle as unicells and only become multicellular under certain environmental conditions. For example, Dictyostelium species can remain in their unicellular state for many generations, without the need to form a multicellular fruiting body, which they do only under certain harsh conditions, and so we classify them as facultatively multicellular. In contrast, in mammals, the unicellular stage is finite and must always ultimately lead to the multicellular stage, and so we classify them as obligately multicellular. Consequently, we are focusing on whether one of the key requirements for a major evolutionary transition in individuality has occurred, termed "contingent irreversibility" $[2,4]$.

We found that species with clonal groups were significantly more likely to have made the transition to obligate multicellularity (Figures 2 and $3 \mathrm{~A}$; pMCMC = 0.0002). Overall, $75 \%$ $(9 / 12)$ of the lineages with clonal group formation had made the transition to obligate multicellularity, whereas the five lineages with potentially nonclonal group formation had only led to facultative multicellularity. Obligate multicellularity is feasible in species with nonclonal group formation-all it requires is that cells always aggregate to complete a necessary part of their life cycle (i.e., not just under certain conditions). Given that we found no evidence for nonclonal organisms having evolved obligate multicellularity, this suggests that the genetic conflict that arises from lower relatedness $(r \leq 1)$ inhibits this major transition in individuality.

If there is a lack of conflict within obligate multicellular groups, such that the group is thought of as an individual, then they will have made a major evolutionary transition in individuality [2-4]. Clonality leads to no within-group conflict [13]. Consequently, because obligate multicellularity has only evolved in species with clonal groups, all of these species have made a major transition in individuality.

\section{Sociality in Multicellular Groups}

We then examined whether relatedness influenced the level of sociality in multicellular groups. We collected data for four lifehistory variables: the number of different cell types that can occur in each group, whether or not species had sterile cells, the percentage of the total number of cells that was sterile in species that had sterile cells, and the total number of cells in the group (organism). The number of different cell types that can occur in a group is analogous to the number of castes in eusocial insect colonies, and hence represents the extent to which different group members specialize in different roles 


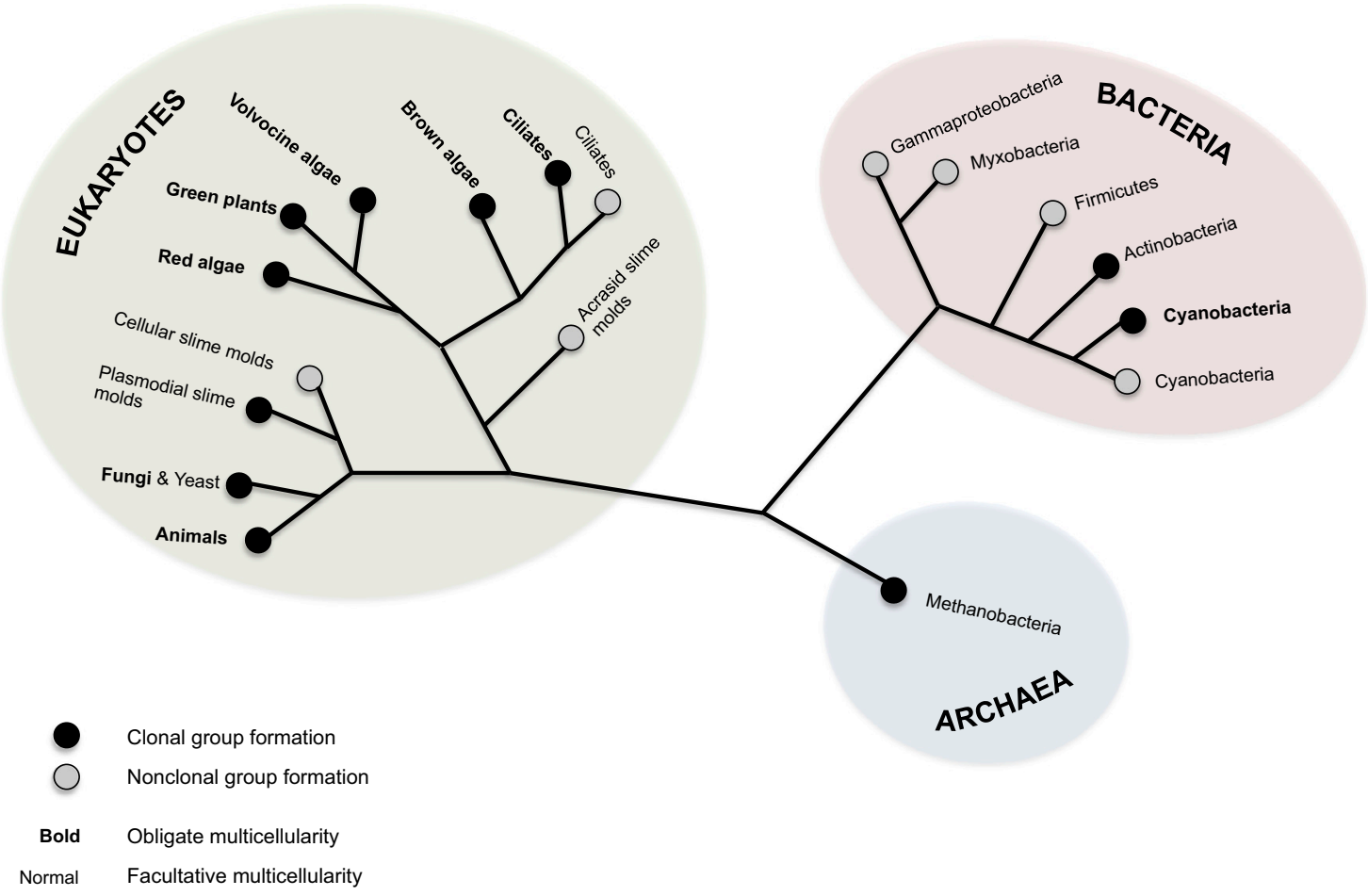

Figure 1. Multicellularity across the Tree of Life

The figure shows an overview phylogram of the taxa in our data set. We have labeled each taxon according to whether multicellular groups are either clonal (black circles) or nonclonal (gray circles) and whether multicellularity is an obligate (bold font) or a facultative (normal font) part of the life cycle. The relatively low number of times that multicellularity has evolved, especially in taxa with nonclonal groups, emphasizes the importance of collecting data on specifically targeted groups to increase the statistical power of phylogenetically based analyses.

[4]. Sterile cells represent a case of extreme altruism, analogous to sterile workers in eusocial insects, forgoing any opportunity to reproduce directly in order to help others [5]. The total number of cells in the group is seen as one of the traits that contribute to and correlate with group complexity [4].

We found that species with clonal groups had significantly higher numbers of cell types and a significantly higher probability of having sterile cells. Species with clonal groups had, on average, approximately six times as many cell types as species with nonclonal groups (Figures 2 and 3B; pMCMC = 0.0008 ) and were approximately twice as likely to have sterile cells (Figures 2 and $3 C$; pMCMC $=0.02$ ). These significant influences of relatedness are particularly impressive given the following: (1) ecological costs and benefits also matter, not just relatedness [17]; (2) relatedness may still be extremely high (for example, $r=0.98$ [20] in the nonclonal groups); and (3) our statistical power is limited by the small number of evolutionary transitions to multicellularity. This emphasizes not only that relatedness matters, but that it matters a lot.

We found that species with clonal groups had a higher proportion of sterile cells (in the species with sterile cells) and a greater number of cells, but that these differences were nonsignificant. In species with sterile cells, those that formed clonal groups had approximately three times the proportion of sterile cells in comparison to species with nonclonal groups (Figure 3D; pMCMC = 0.41). Species with clonal groups had, on average, approximately 100 times as many cells in their group as species with nonclonal groups (Figures 2 and 3E; pMCMC $=0.27$ ). This lack of statistical significance despite the magnitude of differences reflects both high variability and a limited statistical power, due to the small number of independent evolutionary origins of multicellularity, especially those with potentially nonclonal group formation (Figures 1 and $3 \mathrm{~A}$ ). For example, the comparison of the proportion of sterile cells was limited primarily to a comparison of clonal volvocine algae and cyanobacteria with the nonclonal cellular slime molds (Figure 3D). The high variability may reflect that although a higher relatedness can favor more sterile cells and larger groups, the ecological costs and benefits of these traits could vary across taxa.

\section{Causality and Assumptions}

We have examined how relatedness influences both whether a transition to obligate multicellularity has occurred and the different traits that determine the level of sociality, such as whether there are sterile cells in the group. Previous work has examined multiple traits simultaneously by comparing simple and complex multicellular species, where complexity is defined by the presence of sterile cells, an early germsoma split, a high number of cell types, and a large number of cells $[4,10,11]$. However, although the simple/complex distinction is likely to be correlated with whether a major transition has occurred, it is not a defining feature, and the correlation can break down [7]. For example, Dictyostelium purpureum and Volvox carteri are both simple, having a sterile soma and the same number of cell types, but only $V$. carteri has undergone the major transition to obligate multicellularity. Furthermore, although complex multicellularity has evolved only in species with a single-cell stage (unitary development) that leads to clonality $(r=1)[10,11]$, there is no significant correlation between relatedness and whether multicellularity is simple or complex (pMCMC $=0.21$ ). A problem here is that 


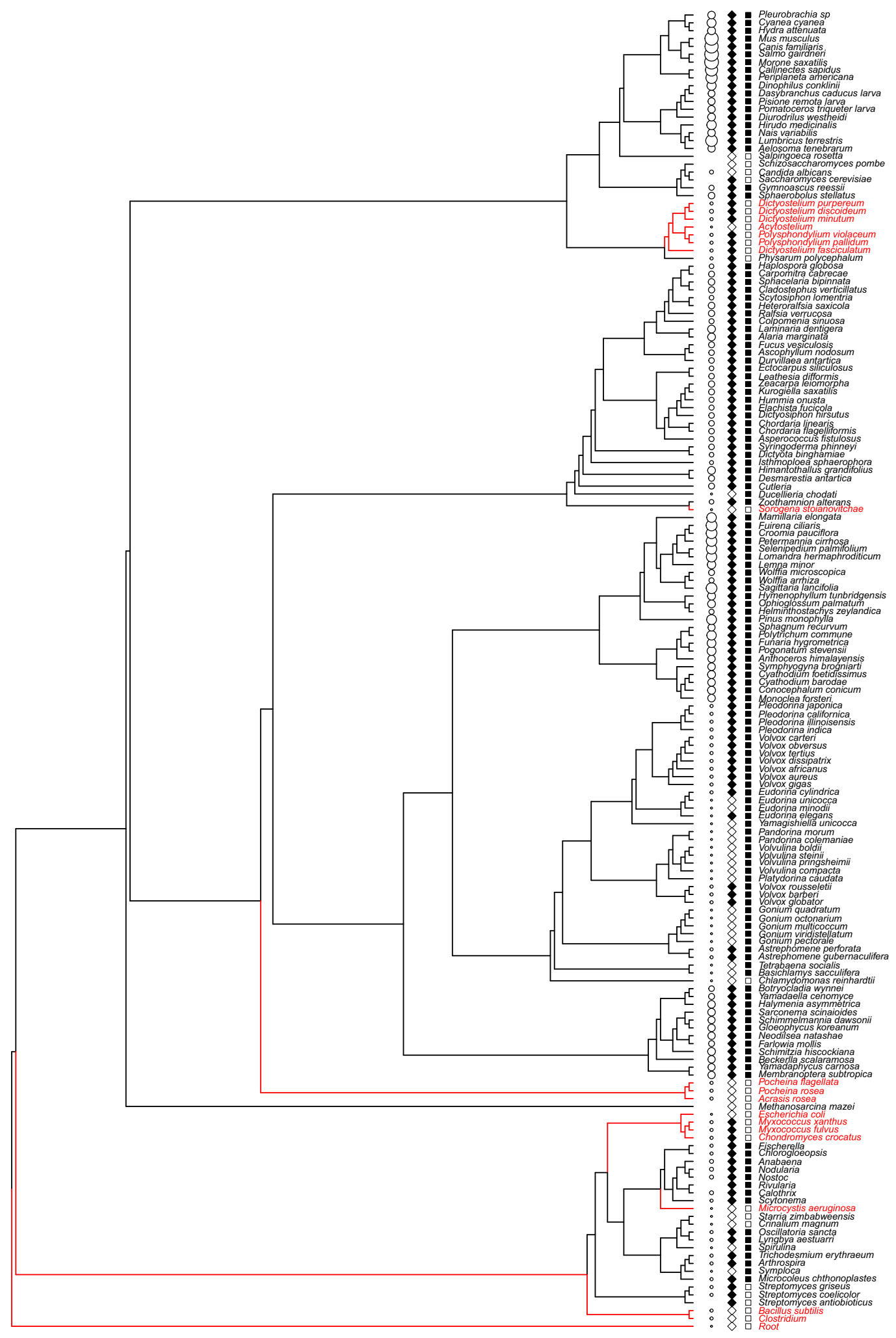

Figure 2. Evolutionary Relationships between Clonality and Multicellular Traits

The figure shows the evolutionary transitions between clonal (black tips and edges) and nonclonal (red tips and edges) group formation and its correlation with obligate (black squares) versus facultative (white squares) multicelluarity, the presence of sterile soma (black diamonds represent sterile soma; white diamonds represent no sterile soma), and the number of different cell types (the size of the white circles represents the natural logarithm of the number of cell types). Ancestral reconstruction of clonal and nonclonal states was conducted using Bayesian phylogenetic mixed models in MCMCglmm (see also Figures S1 and S2). 


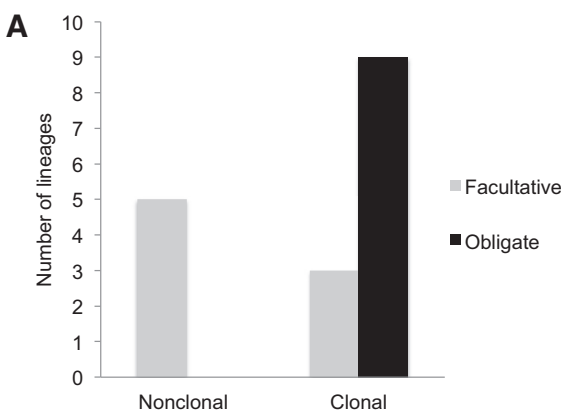

B

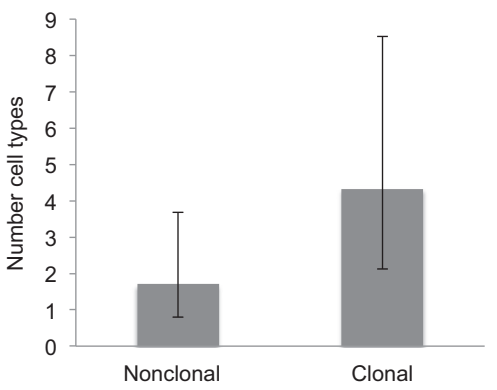

D

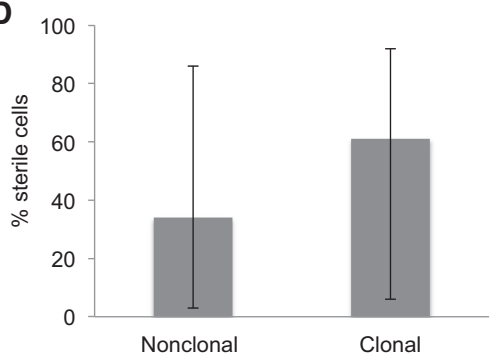

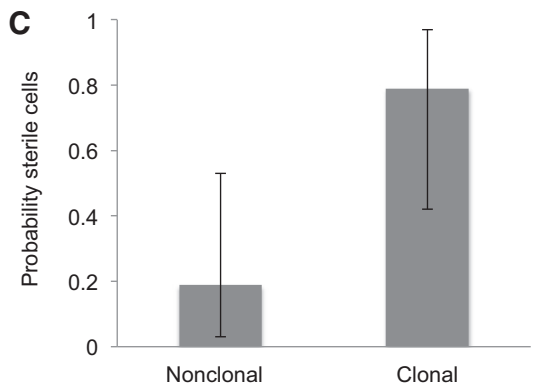

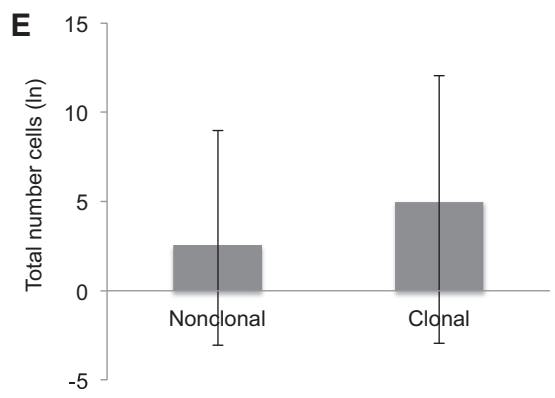

Figure 3. Group Formation, Evolutionary Transitions, and Sociality

The graphs show, for multicellular groups that are either clonal or potentially nonclonal, the (A) number of lineages in which facultative and obligate multicellularity have evolved; (B) number of cell types; (C) probability of having steriles cells; (D) percentage of sterile cells in species with a sterile soma; and (E) total number of cells. Estimates in (B)-(E) are posterior modes with $95 \%$ credible intervals from Bayesian phylogenetic mixed models. The number of cell types is backtransformed from a Poisson distribution with log link function, the probability of sterile cells from a binary distribution with logit link function, the proportion of sterile cells from a binomial distribution with a logit link function, and total number of cells is on a logarithmic scale (see also Figure S3).

developmental history that could facilitate the scope for coordinated development among cells, for example into different cell types [11]. This should not be seen, however, as a competing explanation to relatedness, as it is a mechanistic (proximate) and not a selective (ultimate) issue $[25,26]$. For example, although the potential for coordination could place a limit on the number of cell types (the strategy set open to natural selection), we still need an explanation for why multiple cell types are favored (which strategy is favored by natural selection). Nonetheless, interactions can be important, as complex multicellularity has evolved only five times, in sister groups: animals, fungi, plants, red algae, and brown algae [4, 11]. Consequently, complex multicellularity hasn't evolved in enough separate places on the tree of life to provide statistical power for a formal comparative analysis [19, 21].

Our results are robust for the assumptions that we make about how relatedness and the various social traits coevolve. We assumed that relatedness does not evolve and causally affects each of the social traits. Although the causal effect of relatedness on the evolution of social traits is strongly supported [22], the variation in relatedness among cells may change over evolutionary time. We therefore reanalyzed our data, allowing both relatedness and the social traits to evolve across the tree, while relaxing our assumption of causality [23]. These analyses gave qualitatively identical results, with a significant statistical correlation between relatedness and obligate versus facultative multicellularity $(p<0.0001)$, number of cell types ( $p=0.003$ ), and probability of sterile soma $(p=0.009)$, but not number of cells $(p=0.08)$ or the proportion of sterile cells in the species that had sterile cells ( $p=0.34$; Table S3).

In almost all of the species with clonal group formation, a unicellular stage is involved, wherein the group (or organism) arises from a single cell, usually a zygote or spore $[10,11]$. The exception to this is in species with multicellular propagules, wherein the propagules are formed by cells remaining with their parents, as in some cyanobacteria [24]. Another consequence of a unicellular stage is that it leads to a shared natural selection can only act on the options that are developmentally and mechanistically possible.

\section{Relatedness and the Major Transitions}

More generally, when combined with data from previous studies, our results show how relatedness provides a single life-history variable that plays a key role in explaining evolutionary transitions that involve members of the same species joining together to cooperate $[3,4,7-9,27]$. We have shown how a higher relatedness between cells leads to a higher likelihood of obligate multicellular groups, a higher number of cell types, and a higher likelihood of sterile cells (Figures 2, 3, and S3). A previous experimental study has shown that Dictyostelium discoideum loses cooperative fruiting-body formation if kept under conditions of low relatedness [12]. Previous comparative studies have shown how a higher within-group relatedness is correlated with cooperative breeding in both birds and mammals $[28,29]$ and with the evolution of eusociality in animals [8, 9, 30].

In all cases, relatedness is determined by how groups are formed. A higher relatedness arises from (1) offspring staying with their parents (termed "subsociality") rather than individuals of the same generation aggregating together (termed "parasociality" or "semisociality") and (2) either asexuality or lower levels of promiscuity. Taken together, these results support a fundamental role of how groups form, via the influence of group formation on relatedness, for the evolution of cooperative breeding, eusociality, and now multicellularity $[7-9,28-30]$ 


\section{Sexual Animals}
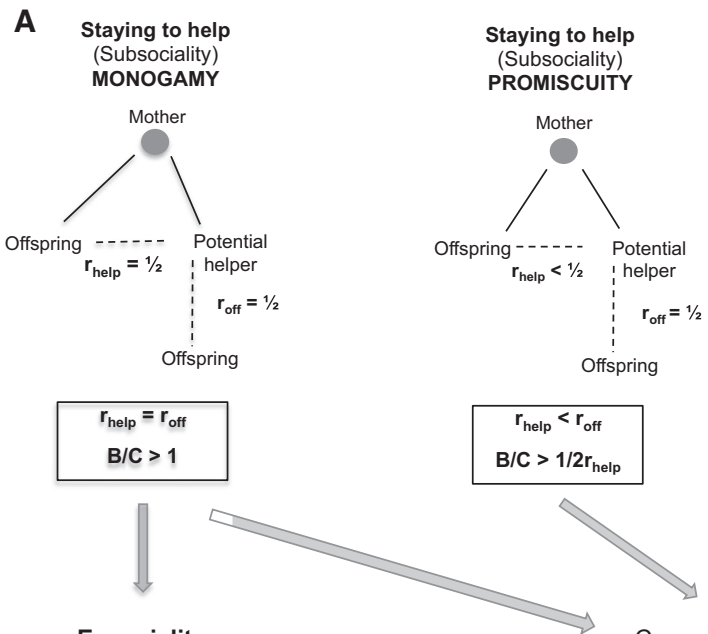

Eusociality
Asexual Animals \& Cells
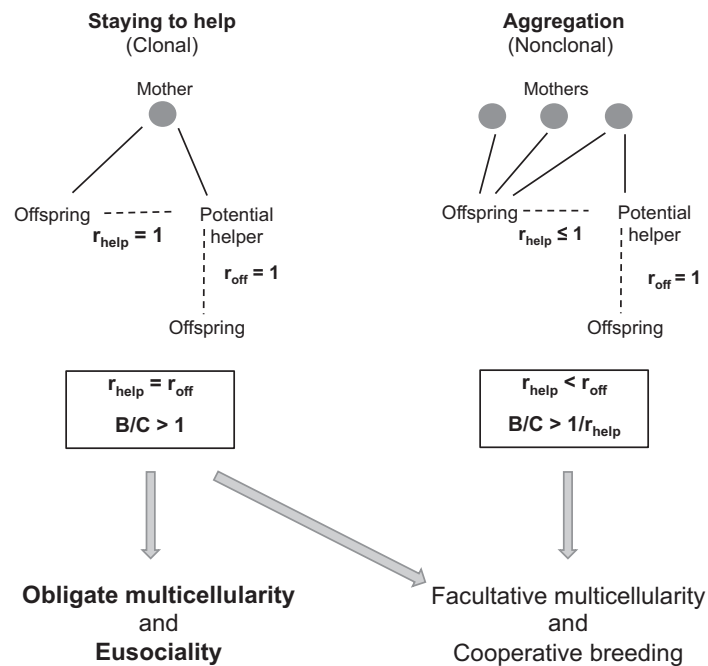

Eusociality
Cooperative breeding

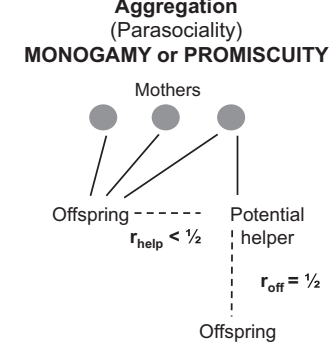

$$
\begin{gathered}
r_{\text {help }}<r_{\text {off }} \\
B / C>1 / 2 r_{\text {help }}
\end{gathered}
$$$$
B / C>1 / 2 r_{\text {help }}
$$

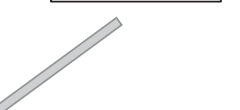

Figure 4. Relatedness, Group Formation, and Evolutionary Transitions

(A) In sexual animals with strict lifetime monogamy, when groups form by offspring staying to help their mother (subsociality), potential helpers are equally related to their own offspring $\left(r_{\text {off }}=1 / 2\right)$ and to their mother's offspring $\left(r_{\text {help }}=1 / 2\right)$, who they could help raise. This means that there is no conflict over which offspring to raise $\left(r_{\text {off }}=r_{\text {help }}\right)$, which facilitates the evolution of reproductive dependency between group members and means that any small efficiency benefit of helping will favor altruistic cooperation $[8,13](B / C>1$, where $B$ and $C$ are the benefit and cost terms in Hamilton's rule [17]). In contrast, if groups form by individuals from the same generation aggregating together (parasociality), such as sisters, or if females mate multiply during their lifetime (promiscuity), then potential helpers are more related to their own offspring $\left(r_{\text {off }}=1 / 2\right)$ than to the offspring who they could help raise $\left(r_{\text {help }}<1 / 2\right)$. In this case, it is much harder to evolve complete reproductive dependency between group members, and a larger efficiency benefit of helping is required to favor cooperation.

(B) The same general predictions occur when considering group formation by cells or asexual animals, such as aphids. Specifically: when groups form by offspring remaining with their parents, this leads to potential helpers being equally related to their own offspring and to the offspring that they could help raise $\left(r_{\text {help }}=r_{\text {off }}\right)$, as they are all clonal $(r=1)$; when groups form by individuals aggregating together, this leads to potential helpers being more related to their own offspring than to the offspring who they could help raise $\left(r_{\text {help }}<r_{\text {off }}\right)$.

reproduction [7-9, 11, 30] (Figure 3A). The distinction between facultative and obligate multicellularity is of equal importance, and it is directly analogous to that between facultative and obligate eusociality $[7,9,34]$.

In contrast, when there is not strict lifetime monogamy in sexual species, or groups form by individuals of the same

(Figures 2 and 3). Experimental evolution studies suggest that the method of group formation is similarly important for a range of cooperative behaviors in microorganisms [31-33].

Furthermore, our data also support Boomsma's hypothesis [7-9] that although a higher relatedness favors greater levels of cooperation, there is something special about clonality in asexual species or strict lifetime monogamy in sexual species. At these extreme points, the offspring that can potentially stay and help their parents are equally related to the offspring they can help raise $\left(r_{\text {help }}\right)$ and the offspring they could produce if they breed independently $\left(r_{\text {off }}\right)$, such that $r_{\text {help }}=r_{\text {off }}$ (Figure 4). Consequently, as long as there is some ecological benefit to cooperation, this removes any conflict over whether to help or breed [13], and thus facilitates the evolution of complete reproductive dependency between group members. The major evolutionary transitions to obligate multicellularity and obligate eusocial societies have only occurred when $r_{\text {help }}=r_{\text {off }}$ holds, due to group formation by offspring staying with parents combined with either strict lifetime monogamy or asexual generation aggregating together, this leads to $r_{\text {help }}<r_{\text {off }}$, and only transitions to facultative multicellularity or cooperative breeding have occurred $[7-9,11,28,29]$ (Figure 3A). Examples of this include the multiclone fruiting bodies of Dictyostelid slime molds, or the slightly promiscuous cooperative-breeding vertebrates. This suggests that $r_{\text {help }}=r_{\text {off }}$ can be a necessary precursor for "fraternal" major transitions that involve cooperation between members of the same species. If $r_{\text {help }}<r_{\text {off }}$, then at some point in their life, potential helpers may do better by breeding independently; therefore, they are selected to retain the flexibility to do so [7-9]. Major transitions require, by definition, a complete reproductive dependency between group members [2, 4], and thus the retention of this flexibility can stall a potential major transition (Figure 4).

Experimental Procedures

We searched the literature for information on the evolution and development of multicellularity in as many different taxa as possible and found data for 17 out of the 25 independent evolutionary transitions to multicellularity 
(Supplemental Information). Several groups (e.g., plants) include both complex and simple multicellular species. When analyzing the evolutionary transitions (Figure 3) we are interested in the highest level of complexity obtained; therefore, we classified each group according to the more complex species in the group. A single-cell (unitary) stage leads to clonality [10,11], but clonality can also occur in species with multicellular propagules when the propagules are formed by cells remaining with their parents, as in some cyanobacteria [24]. This matters because, from an evolutionary theory perspective, the key distinction is whether groups are clonal $(r=1)$ or not $(r<$ 1 ), and not just whether there is a single-cell (unitary) stage (although they will be highly correlated $[10,11]$ ).

We examined whether the evolution of multicellularity was influenced by relatedness by testing whether there were differences between clonal and nonclonal taxa for six different social traits: (1) obligate versus facultative multicellularity (binary distribution); (2) total number of cells (Gaussian after log transformation); (3) the number of cell types after controlling for total number of cells (Poisson distribution); (4) presence of sterile cells (binary distribution); (5) percentage of cells that are sterile in taxa with sterile soma (binomial distribution); and (6) complex versus simple multicellularity (binary distribution). We carried out our analyses using Bayesian phylogenetic mixed models (BPMM) with MCMC estimation implemented in MCMCgImm and checked the robustness of our results to assumptions of causality and tree-branch lengths (Supplemental Information).

\section{Supplemental Information}

Supplemental Information includes Supplemental Experimental Procedures, three figures, and seven tables and can be found with this article online at http://dx.doi.org/10.1016/j.cub.2013.05.004.

\section{Licensing Information}

This is an open-access article distributed under the terms of the Creative Commons Attribution License, which permits unrestricted use, distribution, and reproduction in any medium, provided the original author and source are credited.

\section{Acknowledgments}

We thank Bettina Schirrmeister for comments on cyanobacteria; Koos Boomsma, Kevin Foster, Andy Gardner, Ashleigh Griffin, Rick Grosberg, Lee Henry, Elli Leadbeater, Andy Young, and an anonymous referee for comments on the manuscript; and the ERC, NERC, and Vetenskapsraidet for funding.

Received: February 1, 2013

Revised: April 9, 2013

Accepted: May 1, 2013

Published: June 6, 2013

\section{References}

1. Leigh, E.G., Jr. (1995). The major transitions of evolution. Evolution 49 1302-1306.

2. Maynard Smith, J., and Szathmáry, E. (1995). The Major Transitions in Evolution (New York: Oxford University Press).

3. Queller, D.C. (2000). Relatedness and the fraternal major transitions. Philos. Trans. R. Soc. Lond. B Biol. Sci. 355, 1647-1655.

4. Bourke, A.F.G. (2011). Principles of Social Evolution (Oxford: Oxford University Press).

5. Strassmann, J.E., and Queller, D.C. (2010). The social organism: congresses, parties, and committees. Evolution 64, 605-616.

6. Michod, R.E., and Roze, D. (2001). Cooperation and conflict in the evolution of multicellularity. Heredity (Edinb) 86, 1-7.

7. Boomsma, J.J. (2013). Beyond promiscuity: mate-choice commitments in social breeding. Philos. Trans. R. Soc. Lond. B Biol. Sci. 368, 20120050.

8. Boomsma, J.J. (2007). Kin selection versus sexual selection: why the ends do not meet. Curr. Biol. 17, R673-R683.

9. Boomsma, J.J. (2009). Lifetime monogamy and the evolution of eusociality. Philos. Trans. R. Soc. Lond. B Biol. Sci. 364, 3191-3207.

10. Grosberg, R.K., and Strathmann, R.R. (1998). One cell, two cell, red cell, blue cell: The persistence of a unicellular stage in multicellular life histories. Trends Ecol. Evol. 13,112-116.
11. Grosberg, R.K., and Strathmann, R.R. (2007). The Evolution of Multicellularity: A Minor Major Transition? Annu. Rev. Ecol. Evol. Syst. 38, 621-654.

12. Kuzdzal-Fick, J.J., Fox, S.A., Strassmann, J.E., and Queller, D.C. (2011) High relatedness is necessary and sufficient to maintain multicellularity in Dictyostelium. Science 334, 1548-1551.

13. Gardner, A., and Grafen, A. (2009). Capturing the superorganism: a formal theory of group adaptation. J. Evol. Biol. 22, 659-671.

14. Michod, R.E. (1997). Cooperation and Conflict in the Evolution of Individuality. I. Multilevel Selection of the Organism. Am. Nat. 149, 607-645.

15. Keller, L. (1999). Levels of selection in evolution (Princeton, NJ: Princeton University Press).

16. Frank, S.A. (1995). Mutual policing and repression of competition in the evolution of cooperative groups. Nature $377,520-522$

17. Hamilton, W.D. (1964). The genetical evolution of social behaviour. I. J. Theor. Biol. 7, 1-16.

18. Leigh, E.G., Jr. (1991). Genes, bees and ecosystems: The evolution of a common interest among individuals. Trends Ecol. Evol. 6, 257-262.

19. Hadfield, J.D., and Nakagawa, S. (2010). General quantitative genetic methods for comparative biology: phylogenies, taxonomies and multitrait models for continuous and categorical characters. J. Evol. Biol. 23, 494-508.

20. Gilbert, O.M., Foster, K.R., Mehdiabadi, N.J., Strassmann, J.E., and Queller, D.C. (2007). High relatedness maintains multicellular cooperation in a social amoeba by controlling cheater mutants. Proc. Natl. Acad. Sci. USA 104, 8913-8917.

21. Harvey, P.H., and Purvis, A. (1991). Comparitive methods for explaining adaptations. Nature 351, 619-624.

22. Davies, N.B., Krebs, J.R., and West, S.A. (2012). An Introduction to Behavioural Ecology, Fourth Edition (Oxford: Wiley-Blackwell).

23. Felsenstein, J. (2012). A comparative method for both discrete and continuous characters using the threshold model. Am. Nat. 179, 145-156.

24. Schirrmeister, B.E., Antonelli, A., and Bagheri, H.C. (2011). The origin of multicellularity in cyanobacteria. BMC Evol. Biol. 11, 45.

25. Mayr, E. (1961). Cause and effect in biology. Science 134, 1501-1506.

26. Tinbergen, N. (2010). On aims and methods of Ethology. Z. Tierpsychol. 20, 410-433.

27. Hatchwell, B.J.; Philosophical Transactions of the Royal Society B. (2009). The evolution of cooperative breeding in birds: kinship, dispersal and life history. Philos. Trans. R. Soc. Lond. B Biol. Sci. 364, 3217-3227.

28. Cornwallis, C.K., West, S.A., Davis, K.E., and Griffin, A.S. (2010) Promiscuity and the evolutionary transition to complex societies. Nature 466, 969-972.

29. Lukas, D., and Clutton-Brock, T. (2012). Cooperative breeding and monogamy in mammalian societies. Proc. Biol. Sci. 279, 2151-2156.

30. Hughes, W.O.H., Oldroyd, B.P., Beekman, M., and Ratnieks, F.L.W. (2008). Ancestral monogamy shows kin selection is key to the evolution of eusociality. Science 320, 1213-1216.

31. Griffin, A.S., West, S.A., and Buckling, A. (2004). Cooperation and competition in pathogenic bacteria. Nature 430, 1024-1027.

32. MacLean, R.C., and Gudelj, I. (2006). Resource competition and social conflict in experimental populations of yeast. Nature 441, 498-501.

33. Diggle, S.P., Griffin, A.S., Campbell, G.S., and West, S.A. (2007). Cooperation and conflict in quorum-sensing bacterial populations. Nature 450, 411-414.

34. Crespi, B.J., and Yanega, D. (1995). The definition of eusociality. Behav. Ecol. 6, 109-155. 
Appendix 2: Major evolutionary transitions in individuality 


\title{
Major evolutionary transitions in individuality
}

\author{
Stuart A. West ${ }^{a, b, 1}$, Roberta M. Fisher ${ }^{a}$, Andy Gardner ${ }^{c}$, and E. Toby Kiers ${ }^{d}$

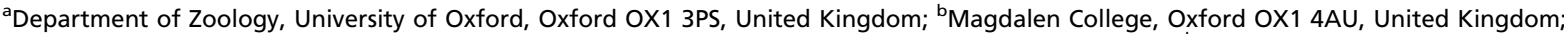 \\ 'School of Biology, University of St. Andrews, Dyers Brae, St. Andrews KY16 9TH, United Kingdom; and Institute of Ecological Sciences, Faculty of \\ Earth and Life Sciences, Vrije Universiteit, 1081 HV, Amsterdam, The Netherlands
}

Edited by John P. McCutcheon, University of Montana, Missoula, MT, and accepted by the Editorial Board March 13, 2015 (received for review December 7, 2014)

The evolution of life on earth has been driven by a small number of major evolutionary transitions. These transitions have been characterized by individuals that could previously replicate independently, cooperating to form a new, more complex life form. For example, archaea and eubacteria formed eukaryotic cells, and cells formed multicellular organisms. However, not all cooperative groups are en route to major transitions. How can we explain why major evolutionary transitions have or haven't taken place on different branches of the tree of life? We break down major transitions into two steps: the formation of a cooperative group and the transformation of that group into an integrated entity. We show how these steps require cooperation, division of labor, communication, mutual dependence, and negligible within-group conflict. We find that certain ecological conditions and the ways in which groups form have played recurrent roles in driving multiple transitions. In contrast, we find that other factors have played relatively minor roles at many key points, such as within-group kin discrimination and mechanisms to actively repress competition. More generally, by identifying the small number of factors that have driven major transitions, we provide a simpler and more unified description of how life on earth has evolved.

cooperation | altruism | signaling | division of labor | conflict

$\mathrm{T}_{\mathrm{p}}^{\mathrm{h}}$ he evolution of life, from simple organic compounds in a primordial soup to the amazing diversity of contemporary organisms, has taken roughly 3.5 billion years. How can we explain the evolution of increasingly complex organisms over this period? A traditional approach has been to consider the succession of taxonomic groups, such as the age of fishes giving rise to the age of amphibians, which gave way to the age of reptiles, and so on. Although this approach has some uses, it is biased toward relatively large plants and animals and lacks a conceptual or predictive framework, in that it suggests we look for different explanations for each succession (1).

Twenty years ago, Maynard Smith and Szathmáry (2) revolutionized our understanding of life on earth by showing how the key steps in the evolution of life on earth had been driven by a small number of "major evolutionary transitions." In each transition, a group of individuals that could previously replicate independently cooperate to form a new, more complex life form. For example, genes cooperated to form genomes, archaea and eubacteria formed eukaryotic cells, and cells cooperated to form multicellular organisms (Table 1).

The major transitions approach provides a conceptual framework that facilitates comparison across pivotal moments in the history of life $(2,3)$. It suggests that the same problem arises at each transition: How are the potentially selfish interests of individuals overcome to form mutually dependent cooperative groups? We can then ask whether there are any similarities across transitions in the answers to this problem. Consequently, rather than looking for different explanations for the succession of different taxonomic groups, we could potentially identify a few key factors that have been important again and again at driving increases in organismal complexity. This approach would both unify and simplify our understanding of the evolution of life on earth.

We define the steps and processes in major transitions and show that the problem of explaining major transitions can be broken down into six questions. We explore what is already known about the factors facilitating transitions, examining the extent to which we can generalize across the different transitions. Ultimately, we are interested in the underlying evolutionary and ecological factors that drive major transitions.

\section{Defining Major Transitions}

A major evolutionary transition has been most broadly defined as a change in the way that heritable information is stored and transmitted (2). We focus on the major transitions that lead to a new form of individual (Table 1), where the same problems arise, in a way that facilitates comparison, and so exclude the evolution of the genetic code, sex and language $(1,2,4,5)$.

A major evolutionary transition in individuality is defined by two conditions $(1,2)$. First, entities that were capable of independent replication before the transition can replicate only as part of a larger unit after it, termed mutual dependence, interdependence, or contingent irreversibility. Second, there is a relative lack of within-group conflict such that the larger unit can be thought of as a fitnessmaximizing individual (or organism) in its own right. For example, it is common to think of the nucleus and organelles of a eukaryotic cell, or the group of cells that makes up a multicellular animal, as a single organism (6).

When these two conditions are met, evolution can lead to a new higher level individual (organism). We have a group that can't be broken up (condition 1), and we can conceptualize the group acting with a single purpose, where the interests of the previously independent individuals are now aligned (condition 2). We provide examples of how this definition can be applied in Table 1. A precise definition matters because a blurring of exactly what is being examined can obscure the relative importance of the underlying selective forces $(7,8)$.

\section{Steps}

Major evolutionary transitions can be divided into two steps: $(i)$ the formation of a cooperative group and (ii) the transformation of the cooperative group into a more cohesive and integrated entity that can be considered a new level of individual (organism) (Fig. 1) (1, 2). The second step typically involves a number of common features, including the following: the individuals in a group evolving to perform different tasks (division of labor); division of labor becoming so specialized that the members of the group become dependent upon each other; and communication to coordinate cooperation at the group level.

Although these two steps may not have clear borders, and can move gradually from one to the next, they can occur in some order (1). For example, in transitions involving members of the same species, we would not expect division of labor to evolve until after cooperative groups have formed. Consequently, the

\footnotetext{
This paper results from the Arthur M. Sackler Colloquium of the National Academy of Sciences, "Symbioses Becoming Permanent: The Origins and Evolutionary Trajectories of Organelles," held October 15-17, 2014 at the Arnold and Mabel Beckman Center of the National Academies of Sciences and Engineering in Irvine, CA. The complete program and video recordings of most presentations are available on the NAS website at www.nasonline.org/Symbioses. Author contributions: S.A.W., R.M.F., A.G., and E.T.K. wrote the paper. The authors declare no conflict of interest.

This article is a PNAS Direct Submission. J.P.M. is a guest editor invited by the Editorial Board.

1To whom correspondence should be addressed. Email: stuart.west@zoo.ox.ac.uk.
} 


\begin{tabular}{|c|c|c|c|c|c|c|}
\hline & & & & & Why $r$ & t? \\
\hline Trans & tion & & Examples & Examples & $\begin{array}{c}\text { Mutual } \\
\text { dependence? }\end{array}$ & $\begin{array}{l}\text { Negligible } \\
\text { conflict? }\end{array}$ \\
\hline Independent replicators & $\rightarrow$ & $\begin{array}{l}\text { Populations of } \\
\text { replicators }\end{array}$ & Genome & - & - & - \\
\hline Separate unicells & $\rightarrow$ & Symbiotic unicell & $\begin{array}{l}\text { Eukaryotic cell*, primary plastid } \\
\text { endosymbiosis*, Paulinella* } \\
\text { Secondary/tertiary plastid } \\
\text { endosymbiosis* }\end{array}$ & - & - & - \\
\hline Unicells & $\rightarrow$ & $\begin{array}{l}\text { Multicellular } \\
\text { organism }\end{array}$ & $\begin{array}{c}\text { Animals, plants, fungi, red } \\
\text { algae, brown algae }\end{array}$ & $\begin{array}{c}\text { Dictyostelid slime molds }{ }^{\dagger} \\
\text { Pseudomonas biofilms }\end{array}$ & $\begin{array}{l}\text { No } \\
\text { No }\end{array}$ & $\begin{array}{l}\text { No } \\
\text { No }\end{array}$ \\
\hline Multicellular organisms & $\rightarrow$ & Eusocial society & $\begin{array}{l}\text { Higher termites, leaf-cutter } \\
\text { ants, honeybee }\end{array}$ & $\begin{array}{c}\text { Social aphids }^{\ddagger} \\
\text { Melipona bees } \\
\text { Cooperative vertebrates } \\
\text { Humans }\end{array}$ & $\begin{array}{l}\text { No } \\
\text { Yes } \\
\text { No } \\
\text { No }\end{array}$ & $\begin{array}{l}\text { Yes } \\
\text { No } \\
\text { No } \\
\text { No }\end{array}$ \\
\hline Multicellular organisms & $\rightarrow$ & $\begin{array}{l}\text { Obligate } \\
\text { interspecific } \\
\text { mutualism }\end{array}$ & $\begin{array}{l}\text { Some obligate endosymbionts } \\
\text { (e.g., Buchnera in aphids*), } \\
\text { leaf-cutter ants and their fungi, } \\
\text { Paracatenula flatworms and their } \\
\text { chemoautrophic symbionts }\end{array}$ & $\begin{array}{c}\text { Legumes-Rhizobia } \\
\text { Squid-Vibrio } \\
\text { Gut microbiota }\end{array}$ & $\begin{array}{l}\text { No } \\
\text { No } \\
\text { No }\end{array}$ & $\begin{array}{l}\text { No } \\
\text { No } \\
\text { No }\end{array}$ \\
\hline
\end{tabular}

*Highly integrated/obligate symbioses, where the hosts cannot survive without their symbionts, and there seems to be a relative lack of conflict, such that the symbionts can best increase their own fitness by helping their hosts reproduce (45).

${ }^{\dagger}$ Facultatively multicellular species, which can remain in their unicellular state for many generations, without the need to form a multicellular fruiting body, which they do only under certain harsh conditions to increase dispersal success (no mutual dependence) (37). Sufficient conflict to select for noncooperative cheats, and within-group kin discrimination $(68,69)$.

${ }^{\ddagger}$ Facultatively eusocial species, where queens are still able to breed if the workers/soldiers are removed (no mutual dependence).

${ }^{\S}$ Hosts are able to grow and replicate without these symbionts (no mutual dependence), and there is sufficient conflict to select for noncooperative cheats (69).

benefits of having different cell types in multicellular organisms may not provide an explanation for why multicellular groups initially formed. In contrast, with transitions between members of different species, individuals can be specialized to perform different tasks before the formation of cooperative groups, and group transformation involves specialization to help each other.

\section{What Are the Big Questions?}

We suggest six questions that are key to understanding the evolutionary and ecological drivers of major transitions:

i) What conditions favor the formation of cooperative groups?

ii) What conditions maintain cooperation during group transformation?

iii) What conditions favor division of labor?

iv) What conditions favor communication that coordinates cooperation at the group level?

v) What conditions lead to negligible conflict within groups?

$v i$ ) What conditions favor mutual dependence?

Question $i$ concerns the first step in a major transition (the formation of a cooperative group) whereas the other five questions concern the second step in a major transition (the transformation of the cooperative group into a new level of individuality) (Fig. 1). We have divided these questions to identify specific research problems. Our division emphasizes that major transitions are not just about cooperation or extreme altruism (sterility); they are about an extreme form of cooperation, involving multiple traits, division of labor, mutual dependence, and a lack of conflict.

\section{What Conditions Favor Cooperation?}

The first step in a major transition is that individuals come together and form a cooperative group. Cooperation then needs to be maintained while group transformation takes place. We consider the factors that may favor the formation and maintenance of cooperative groups together because the same selective forces can be involved. A trait is cooperative if it provides a benefit to another individual and has evolved at least partially because of that benefit (8). The problem of cooperation is that, all else being equal, cooperators could be exploited and outcompeted by noncooperators (cheats), who gain the benefits from the cooperation of others, but avoid the cost of cooperating (9). The evolution of cooperation requires two conditions $(1,10-12)$. First, there is some ecological or efficiency benefit to cooperation. Second, there is some mechanism that leads to the benefits of cooperation being directed back to the cooperator and/or their relatives.

Ecological or Efficiency Benefits. The ecological benefits of cooperation have been well studied in many taxa. Cells form cooperative multicellular clumps to evade predators, make more efficient use of factors that are excreted from individual cells, and form fruiting bodies that aid dispersal $(1,13)$. Cooperation

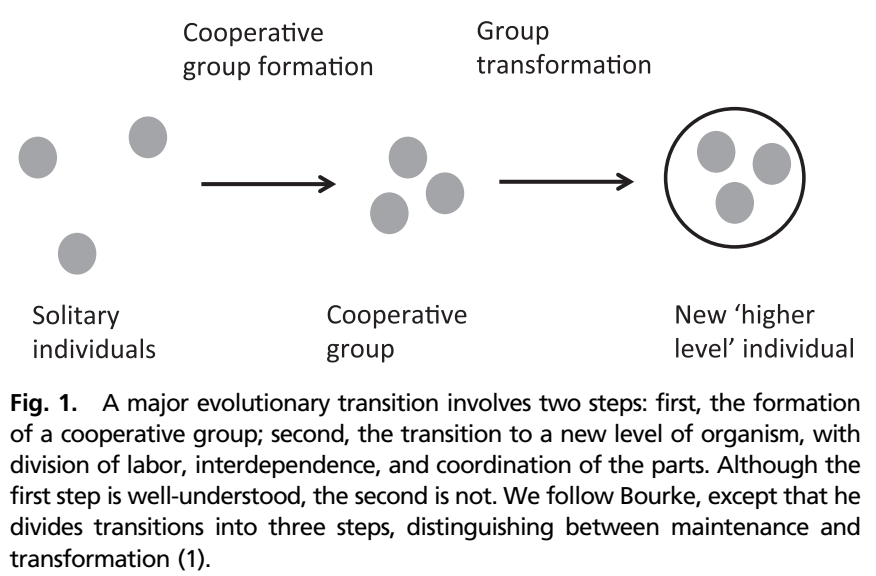




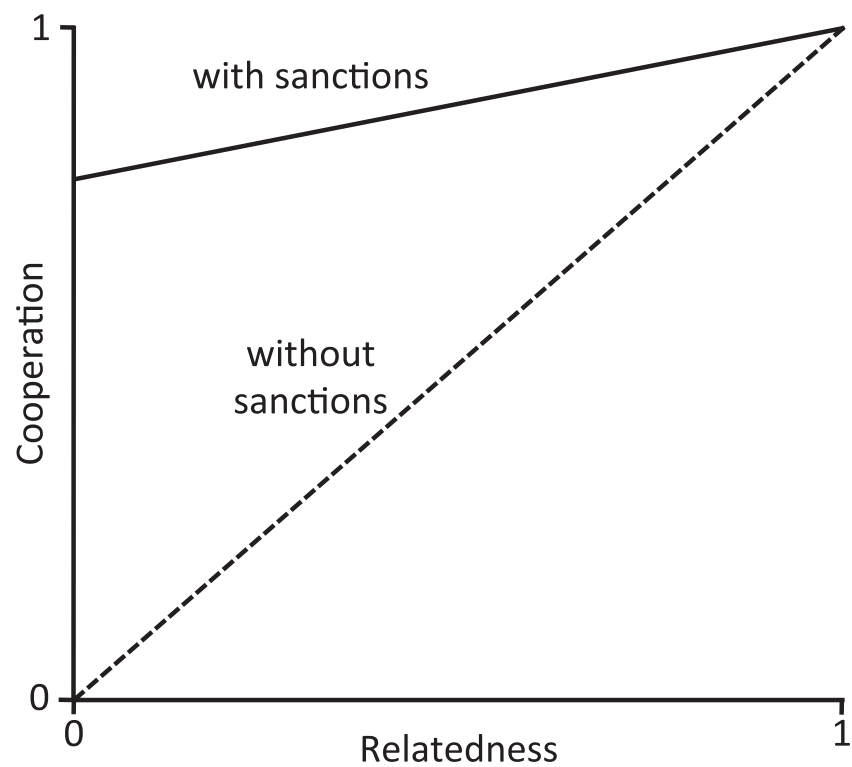

Fig. 2. The hypothetical level of cooperative helping in a symbiont plotted against the relatedness between the symbionts infecting a host. If the hosts sanction uncooperative symbionts, then a high level of cooperation is predicted, relatively independent of relatedness. If the hosts do not carry out sanctions, then the level of cooperation is predicted to depend strongly upon relatedness between symbionts (23).

between different species often involves a symbiont providing a service, such as a resource that allows their host to grow better (14-16). Cooperation is favored in eusocial species to care for young over an extended period where the parent may die, or to defend a valuable resource (17).

Mechanism to Direct Benefits Back (Within Species). Cooperation can be favored if the benefits go to relatives who carry the same genes for cooperation, termed kin selection (18). By helping a close relative reproduce, an individual is still passing on copies of its genes to the next generation, just indirectly. Kin selection is often encapsulated with Hamilton's rule (18), which predicts that altruistic cooperation will be favored when $r B-C>0$, where $C$ is the fitness cost to the altruist, $B$ is the fitness benefit to the beneficiary, and $r$ is their genetic relatedness. Hamilton's rule shows that altruism can be favored when the indirect benefits of helping relatives $(r B)$ outweigh the direct costs $(C)$. There is a large empirical literature supporting the role of relatedness and showing how a high relatedness can arise either through limited dispersal, which keeps relatives together, or kin discrimination, where cooperation is preferentially directed toward relatives $(1,11)$.

Cooperation can be favored between nonrelatives in the same species if it provides a direct benefit to the cooperator: for example, when unrelated ants cooperate to increase their chance of founding a new colony (19). It can also occur via mechanisms, such as reciprocity, that reward cooperators or punish noncooperators (20). In these cases, the costs incurred by cooperating are outweighed by the benefits received in return, and so cooperation is mutually beneficial rather than altruistic.

Mechanism to Direct Benefits Back (Between Species). Cooperation between species also requires mechanisms that lead to the benefits of cooperation being directed back to the cooperator and/or their relatives (21). Broadly speaking, two mechanisms are likely to be important for producing this feedback. The first mechanism is if individuals tend to be associated in such a way that their fitness becomes entwined (partner-fidelity feedback) (12). For example, in a vertically transmitted clonal symbiont, then helping the host could increase the number of host offspring that the symbiont would be transmitted to. Relatedness among the symbionts matters because it determines who receives the benefits of helping the host, and thus whether cheats could exploit the cooperation (22) (Fig. 2). A high relatedness among vertically transmitted symbionts in a host is likely to be important with mitochondria, plastids, and endosymbionts such as Buchnera.

The second mechanism is if the host preferentially rewards more cooperative symbionts and/or punishes less cooperative symbionts (23). For example, legumes provide more resources to more cooperative rhizobia, and squid eliminate symbiotic bacteria that do not luminesce $(24,25)$. Such "sanctions" mechanisms can favor cooperation even when symbiont relatedness is low at the level of the host (Fig. 2).

\section{What Conditions Favor Division of Labor?}

Organisms must perform a number of tasks to survive and reproduce. When will natural selection favor a division of labor, with different individuals performing different tasks? To illustrate this problem with a specific example, consider two tasks, A and $\mathrm{B}$. We assume that investment into these two activities must be traded off against each other because time and energy spent on A cannot be spent on B. Division of labor will tend to be favored when the shape of the relationship between the proportion of resources allocated to each task and the fitness return are accelerating (Fig. 3) (2). The fitness return might be accelerating if a task becomes more efficient as more effort is put into it, or if tasks A and B don't mix well. In contrast, if the fitness returns on tasks are decelerating, then it can be more efficient to have all individuals perform some $\mathrm{A}$ and some B.

There are numerous examples of division of labor. In some volvocine algae, there is a division between large cells that reproduce and small cells who beat their flagella to keep the colony afloat (5). In some cyanobacteria, there is a division between cells that photosynthesize and cells that fix nitrogen into ammonia (heterocysts) - this division seems to be favored because nitrogenase, the enzyme that converts nitrogen gas to ammonia, is rapidly destroyed in the presence of oxygen (26). Symbioses by definition involve a division of labor, with partners providing different services for each other. In some cases, one partner will provide a service that the other partner cannot perform, such as the intracellular chemoautotrophic bacterial symbionts that provide nutrition for marine flatworms lacking a digestive tract (27). Hosts can sometimes harbor multiple symbionts, with different symbionts performing different tasks (28). Mitochondria

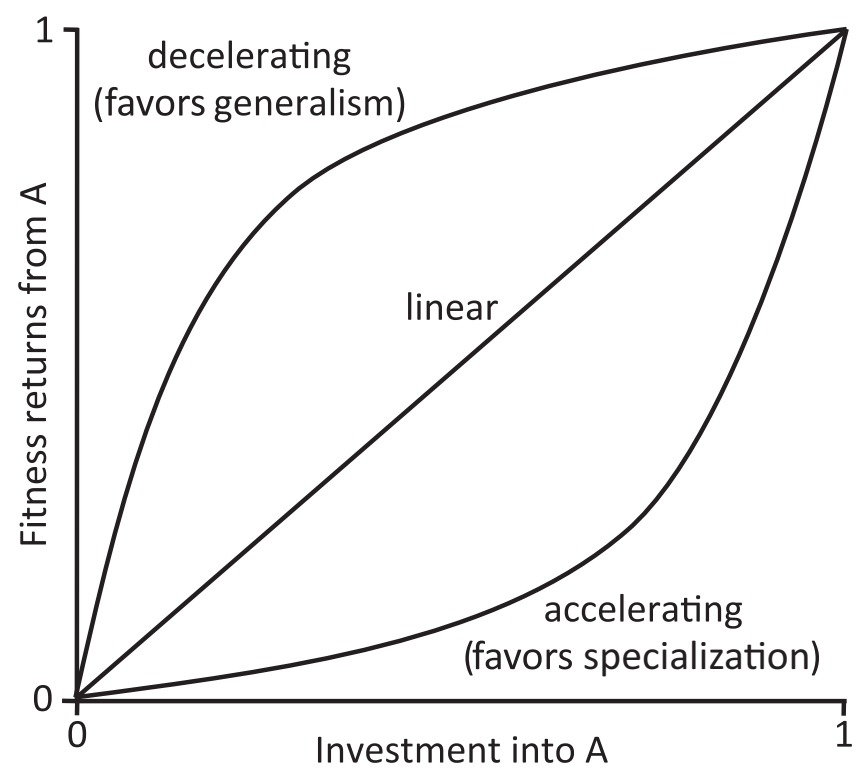

Fig. 3. The relationship between the proportion of resources invested into a trait $(A)$ and the fitness return from that trait. We assume that a proportion of resources $X$ is put intro trait $A$, and the remaining proportion $1-X$ into trait $B$. 
and plastids provide clear examples of extreme specialization in transitions between species.

The general principle is that a division of labor will be favored when there are efficiency benefits to specialization (Fig. 3). There is a lack of research showing why division will be the favored in specific systems. Both theoretical models demonstrating how trade-offs between different traits can lead to benefits of specialization, and empirical studies measuring these trade-offs, are required to address this gap (29). Furthermore, our discussion has ignored many complexities. For example, how would factors such as within-group conflict, variation in relatedness, or different abilities to coordinate at the group level influence selection for division of labor?

\section{What Conditions Favor Communication That Coordinates Cooperation at the Group Level?}

Communication can be key to all stages of a major transition, playing multiple roles in the formation and transformation of cooperative groups: for example, coordinating cooperation and the division of labor at the group level. However, communication systems are potentially exploitable by cheats who fail to respond to signals or who signal dishonestly (30). Individuals could ignore signals to cooperate or could signal in a way to make others cooperate more. What maintains the honesty of the communication systems that help drive major transitions?

Theory has shown that honest signaling requires either that signals can't be faked (indices), that signals are costly to fake (handicaps), and/or that the sender and receiver have a common interest (30). A common interest is particularly relevant in major transitions because the same conditions that favor cooperation can also favor honest communication-high relatedness or aligned reproductive interests. The importance of high relatedness in favoring honest signaling within cooperative groups has been demonstrated experimentally with quorum sensing (QS), the process whereby bacteria use small signal molecules to regulate the production of extracellular factors that aid growth, motility, and biofilm formation. QS is favored at high relatedness and disfavored at low relatedness (31). Common interest presumably explains a range of signals in cases where major transitions have been made, from the waggle dance of the honeybee to among cell coordination in animals. Comparative studies could examine how communication systems vary across species that differ in their degree of conflict.

Symbioses involve many forms of communication, including the acquisition of symbionts from the environment, to the coordination of tasks. Specific cases range from partnerships in which effective communication allows both partners to prosper, to cases in which signals produced from one partner are used to manipulate the other for selfish gain. With luminous bacteria and their squid hosts, there seems to be a mutual interest in coordinating rhythms such that bacterial luminescence peaks when hosts most need the camouflage provided by the bacteria. This coordination involves the host genes' being regulated by the light and molecules produced by the bacteria (32). In other cases, conflict among partners is higher, and signals may be used to manipulate others.

\section{What Conditions Lead to Negligible Conflict Within Groups?}

Even when there are clear benefits to evolving cooperation, conflicts can still arise in cooperative groups. Evolutionary theory predicts that individuals are adapted to maximize their inclusive fitness, even if this maximization comes at a cost to other individuals or the group (18). Inclusive fitness is the sum of an individual's influence on its own reproductive success and that of related individuals. The question here is, as required for a major transition, what conditions are required for individuals maximizing their own inclusive fitness to also be maximizing the fitness of the group (33) (Fig. 4). We focus on inclusive fitness because it is under the full control of the actor, and so represents a measure of fitness that could be maximized (34). It is useful to consider within- and between-species transitions separately.

Within-Species Transitions. What do the empirical data show? Group formation seems to play a key role in whether a major transition to multicellularity or eusociality can take place (Fig. 5)
(1, 35-37). The major transition to obligate multicellularity has taken place only in species where daughter cells stick together after division and so group formation is clonal, such as the animals, fungi, red algae, green plants, volvocine algae, brown algae, some ciliates, and some cyanobacteria $(37,38)$. Although nonclonal group formation occurs via aggregation in many species, such as the cellular slime molds, ciliates, and acrasid slime molds, and can lead to very high relatedness (39), it has led only to facultative multicellularity.

Similarly, the transitions to both facultative and obligate eusociality have taken place only in species where offspring stay to help their parents and there is either strict lifetime monogamy or asexual reproduction $(35,36,40)$. Multiple mating and multiple queens occur in some eusocial species, but they are derived states that evolved after eusociality was fixed. Although cooperation occurs in many species where females mate multiple times, such as the cooperative breeding birds and mammals, no multiply mating species has made a major transition to obligate eusociality (41).

Group Formation. How groups form is important because of its influence on relatedness and conflict. Consider a focal individual who is choosing either $(i)$ to perform a cooperative behavior that helps another individual produce $B$ offspring, to whom the focal actor is related $r_{h}$, or (ii) to produce $C$ of their own offspring to whom they are related by $r_{o}$. In this case, helping is favored if $r_{h} B-r_{o} C>0$, which represents a form of Hamilton's rule. However, we are interested not in whether helping is favored, but whether there is conflict between different individuals over whether to help. Conflict occurs when individuals disagree about the best strategy (6).

What conditions would be required to remove conflict between the helpers (workers) in a social group, such that they could be considered a single maximizing unit? One way to remove this conflict would be if the potential helper is equally related to the offspring that it could help raise and its own offspring $\left(r_{h}=r_{o}\right)(33$, 35-37). In this case, the different offspring are worth equal value to the potential helper, and they are selected to do whatever leads to

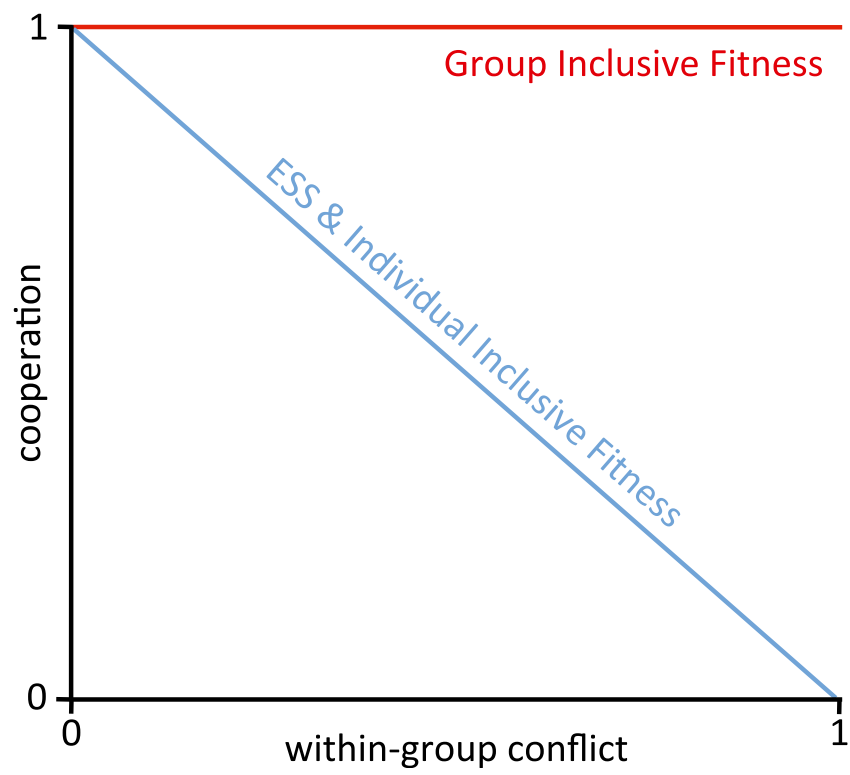

Fig. 4. The individual and the group. The hypothetical level of a cooperative trait, such as the amount of an extracellular factor produced by bacterial cells, plotted against the extent of conflict between interacting individuals. The different lines show the optimal strategy from the perspective of an individual's inclusive fitness (blue line) and group fitness (red line). Natural selection will lead to the evolutionarily stable strategy (ESS), which will be the strategy that maximizes inclusive fitness (i.e., the blue line), irrespective of the consequences at the group level. We would expect natural selection to lead to maximization of group fitness, and thus think of the group as a fitness-maximizing individual, only in extreme cases where there is no within-group conflict. 


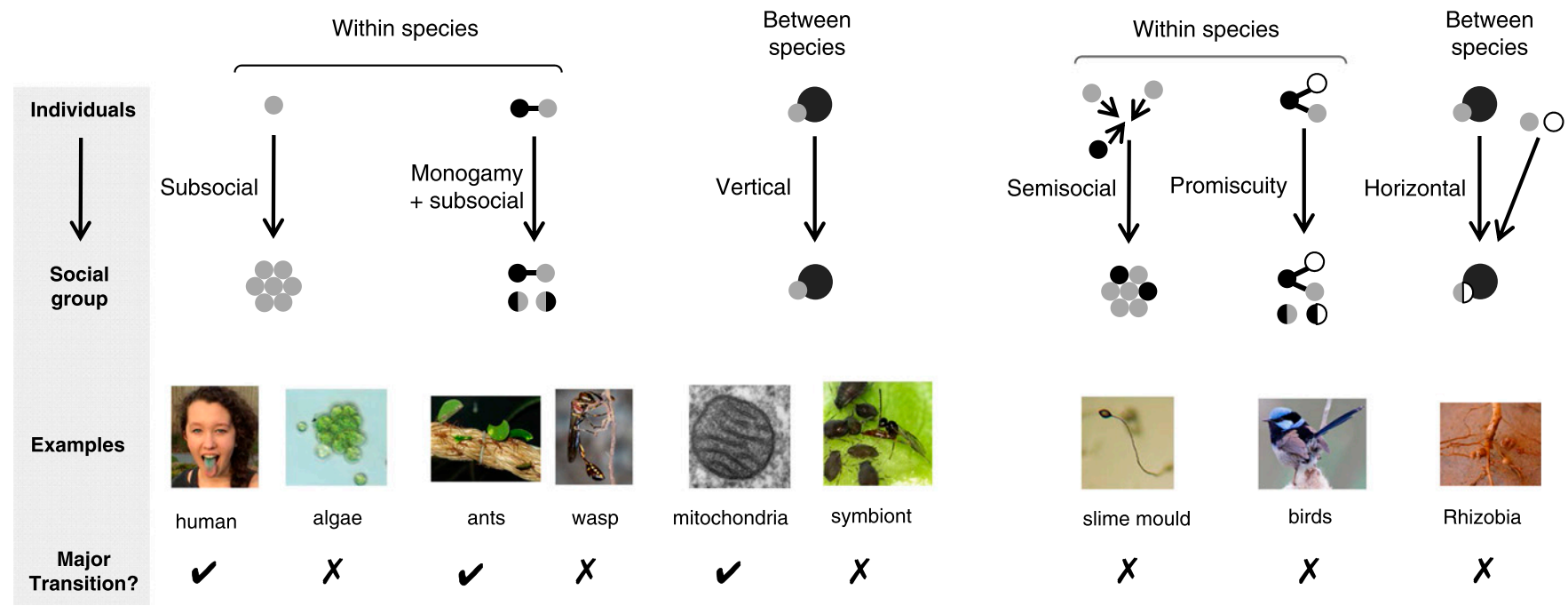

Fig. 5. The way in which groups form is a major determinant of when major transitions have taken place, because it determines relatedness and the potential for within-group conflict. Within-species transitions have taken place only when offspring stay to help their parents (subsocial), and reproduction is either asexual or sexual with lifetime monogamy. Between-species transitions seem to involve similarly restrictive group formation, such as vertical transmission leading to clonal symbionts whose interests are aligned with their hosts. Different colored circles represent either genetically distinct individuals (within-species) or individuals of different species. Larger circles represent hosts with smaller circles representing their symbionts. The images, from left to right show: obligately multicellular human (image courtesy of Stu West), facultatively multicellular Chlamydomonas algae (image courtesy of Will Ratcliff), obligately eusocial Atta ants (image courtesy of Wikimedia commons/Arpingstone), facultatively eusocial Stenogastrine hover wasp (image courtesy of Wikimedia commons/David Baracchi), mitochondrion (image courtesy of Wikimedia commons/Louisa Howard), Hamiltonella defensa symbiont in black bean aphids (image courtesy of Christoph Vorburger), facultatively multicellular Dictyostelium slime mould (image courtesy of Wikimedia commons/Bruno in Columbus), cooperatively breeding superb fairy wren (image courtesy of Wikimedia commons/JJ Harrison), Legume-Rhizobia mutualism (image courtesy of Dave Whitinger, All Things Plants). Negligible conflict is not sufficient for a major transition-the algae, wasp, and symbiont examples have not made a major transition because there is not mutual dependence (see also Table 1).

the production of the most offspring, which will depend upon $B$ and $C$. In a sexual species, this condition is met with strict lifetime monogamy, where potential helpers are aiding their parents, such that they are helping rear full siblings $\left(r_{h}=r_{o}=1 / 2\right)$. In an asexual species, these conditions arise in clonal groups $\left(r_{h}=r_{o}=1\right)$, which also removes conflict between helpers and their parent. Multicellularity and eusociality have evolved only under these conditions.

Our above discussion makes clear that we need to specify whom we are considering potential conflict between, and their behavioral options. We have considered conflict among helpers, and not between helpers and their parents. This assumption is reasonable in large colonies where the workers are effectively in control, but not in smaller colonies or when the reproductives can still wield power over factors such as caste or sex ratio. Consequently, factors such as colony size and caste determination can influence whether a major transition can be made because they will determine the impact of conflict between queens and workers at the colony level.

Conflict can still arise in monogamous species, when the decision a helper faces is not whether to help raise full siblings. In Melipona bees, conflict arises as to whether individuals rear their own offspring, or their niblings (nieces and nephews), resulting in a wasteful 5-14\% of individuals competing to become queens (42). A general issue here is that the Hamilton's rule approach is relatively heuristic, and explicit theory is required to determine when different individuals within a social group will act as a single maximizing agent (33).

Repression. Another way to eliminate within-group conflict is if there is complete repression of competition within groups, such that individuals cannot increase their reproductive success via any form of cheating $(33,43)$. As with the relatedness scenario described above, repression of competition unites the interest of the group. Repression of competition has been important in some social hymenoptera, where workers destroy or "police" the eggs laid by other workers (44). For example, in the honeybee, worker policing is so efficient that workers are effectively selected to not try to reproduce.

Haplodiploidy. Our above discussion of eusociality ignored the complication of haplodiploidy. In diploids, when offspring stay to help their monogamous parents, the helpers are equally related to offspring that they could help raise and their own offspring $\left(r_{o}=r_{h}=1 / 2\right)$. This same condition holds in haplodiploids if helpers cannot differentiate between male and female offspring. However, if we allow for the possibility that males and females can be distinguished from each other, then we find that workers are more related to $(i)$ their own sons $(r=0.5)$ than their brothers $(r=0.25)$, (ii) their sisters $(r=0.75)$ than their daughters $(r=0.5)$, (iii) their daughters $(r=0.5)$ than their brothers $(r=0.25)$, and (iv) their nephews $(r=0.375)$ than their brothers $(r=0.25)$. These relatedness asymmetries can lead to conflicts between workers in haplodiploid species where diploidy would not. Consequently, haplodiploidy hinders major transitions, such that haplodiploid species can require an extra step. Monogamy and sufficient ecological benefit of cooperation can take species to obligate eusociality $(35,40)$ and, in the case of diploids, a major transition. However, then, in haplodiploids, an extra step is required to suppress conflict and complete a major transition: for example, the evolution of multiple mating leading to worker policing (44). This point emphasizes that, in haplodiploids, mating frequency can have different roles at different stages, with monogamy required to make the transition to obligate eusociality, but then multiple mating driving from eusociality to a major transition.

Transitions Between Species. Less attention has been paid to what conditions lead to reduced within-group conflict in betweenspecies transitions. Again, the way in which groups form may be key. There will be no conflict between vertically transmitted clonal symbionts, who could transmit to more individuals only by increasing the reproductive success of their host. Examples that 
seem to lack appreciable conflict include mitochondria, plasmids, the various secondary and tertiary plastid endosymbioses, and possibly the Buchnera bacteria that infect aphids $(14,45)$. In contrast, both the opportunity for horizontal transmission, and within-host symbiont diversity, could lead to conflicts that select for less cooperative symbionts $(22,46)$. Consistent with the predicted role of transmission route, the evolutionary transition from a parasitic to a mutualistic lifestyle in a range of bacterial lineages is associated with the loss of horizontal transmission (47).

Repression of competition could play a role in transitions between species. Sanction mechanisms seem to be more common in partnerships when symbionts transmit horizontally, where we predict that there will be more conflict to resolve, such as in partnerships with root symbionts, luminous symbionts, and pollinator mutualisms $(24,25,48,49)$.

Hosts could structure or transmit their symbionts in a way that better aligns their interests and removes conflict. However, mechanisms that repress competition, such as reducing symbiont diversity, will be favored only if they have an immediate fitness benefit, and not because they will select for more cooperative symbionts in the future (46). For example, leaf-cutter ants actively remove foreign mycelial fragments to prevent incompatibility interactions that can reduce fungal-garden productivity; this removal has the byproduct benefit of more closely aligning the interests of the ants and their fungi over evolutionary time (50). A general problem here is that we lack formal theory for the conditions required to eliminate conflict in between-species transitions.

\section{What Conditions Favor Mutual Dependence?}

In many cases, if a cooperative group is broken up, then individuals are still able to reproduce. In contrast, cases can arise in which different members of the group have lost the capacity for independent replication they once had. For example, mitochondria cannot reproduce without the rest of the eukaryotic cell, and higher termite queens cannot reproduce without the help of their workers. In most cases, mutual dependence seems to arise as a result of extreme division of labor. Individuals become so specialized that they lose the ability to perform other tasks. The question here is what conditions favor the loss of any potential to perform certain tasks that are necessary to replication? Why did higher termite queens lose the ability to feed/rear their offspring, and their workers lose the ability to produce offspring? There is an almost complete lack of work addressing why certain traits are lost, both generally and for specific cases (51).

Presumably, there is some efficiency benefit from becoming irreversibly specialized, which will depend upon a range of biological factors, such as how groups are formed (relatedness), mortality rates, and how those that reproduce (breeders) are replaced. Symbionts offer excellent opportunities for comparative studies on the evolutionary and ecological correlates of mutual dependence. For example, why does the interaction between photosynthetic symbionts and their hosts vary from the obligate symbiosis typified by plastids in plants to ciliates that can "culture" the chloroplasts found in their algal food $(45,52,53)$ ? Are partnerships in which symbionts access and deliver new forms of energy for their hosts more likely to lead to major transitions than cases where the symbionts provide a resource that the host can also obtain directly? Repression of competition can also favor mutual dependence, as demonstrated by the influence of worker policing on whether hymenopteran workers develop ovaries (44).

Genetic drift can play a role in the evolution of mutual dependence. When symbionts have small asexual populations, drift can be a significant factor, leading to the inactivation and eventual deletion of mildly beneficial genes (54). As symbionts lose functionality in traits or structures, such as transporters and cell walls, the host may evolve to take over these roles, coadapting in a way that leads to mutual dependence. Similarly, hosts can lose traits that the symbionts perform. Oligochaete worms have lost the ability to excrete their own waste, relying instead on metabolic pathways introduced by endosymbionts to provide and process nutrients (55), whereas coral hosts (e.g., Acropora sp.) have lost their cysteine synthesis pathway in favor of symbiont-derived sources (56). When multiple symbiont lineages are within a host, different lineages may lose different traits, such that multiple symbionts and the host can become interdependent (28).

\section{Conflict and Maximizing Agents}

A major transition requires that there is a relative lack of withingroup conflict such that a larger unit can be thought of as a fitness-maximizing agent (individual or organism) in its own right. We are interested in when a group can be considered a fitness-maximizing agent for at least three reasons $(6,34)$. First, the maximizing agent analogy informs us when group-level adaptation will occur (33). Almost all interesting traits and behaviors are the construction of multiple entities (genes, cells, etc.), pulling in the same direction cooperatively with minimal conflict. If there is negligible conflict, and we can think of a group as a single maximizing agent, then we can expect adaptation at the group level to maximize the fitness of the group and, thus, substantial increases in organismal complexity. For example, clonal multicellular groups have led to animals and plants whereas nonclonal multicellular groups have led only to things like slime molds.

Second, this analogy facilitates progress at the interface of theory and data in evolutionary biology (34). It allows us to think about a single, individual-level agent, rather than trillions of cellor gene-level agents (57). By black-boxing these lower layers, we can focus on other aspects of biology, such as ecology and behavior, making it easier to develop models and test the robustness of those models to changes in the underlying biological parameters. Nonetheless, the maximizing agent analogy is a heuristic approach that should be used only when the advantages outweigh the disadvantages, which is best judged empirically (58). Third, a focus on maximizing agents makes it easier to identify potential conflicts and determine how they are resolved, both between and within individuals: for example, how conflict can arise over who produces male offspring in haplodiploid social insects, and how conflict is suppressed by worker policing (44).

More generally, we emphasize that the fundamental question being asked with major transitions is one of individuality, and not other issues such as sterility, altruism, complexity, ecological impact, or whether gene transfer has occurred (33). Although traits such as sterility raise important evolutionary questions and can be correlated with whether a major transition has occurred, they are neither necessary nor sufficient for a major transition. Indeed, other examples of complete altruism can be found, which are clearly not major transitions, including bacteria bursting suicidally to release factors that reduce competition (59).

\section{Pragmatism}

Identifying whether a major transition has been made is an empirical problem, where pragmatism can be important. From a theoretical perspective, we can identify the ideal qualities that would unequivocally define individuality and identify when a major transition has been made. For example, we can examine what conditions would lead to no conflict, such as clonality or complete repression of competition (33). In the real world, conflict between genes or cells can still exist. For example, in animals and plants, there is still some opportunity for conflict among genes, and somatic mutation can lead to conflicts among cells $(6,60,61)$. Our aim is to consider when there is so little conflict that the consequences for the group can be effectively ignored.

Whether there is negligible conflict and the group acts as a fitness-maximizing agent can be trait-dependent, even within an organism. For example, although we expect plant traits controlled by nuclear genes to maximize plant fitness, we know that cytoplasmic genes can select for male sterility, to their own selfish benefit. Similarly, in ants such as Formica exsecta, we can assume that the different ants are behaving as a single maximizing unit for traits such as foraging, refuse disposal, etc., whereas there is also clear conflict between the queen and the workers over sex allocation (62). What is key is not to argue whether a certain species has made a major transition, but to use the approach in a way that helps us understand the processes that lead to major transitions: for examining what conditions 
lead to greater mutual dependence or lower conflict. Evolution is a process of continuous change, and so we should expect blurry edges with a mosaic of features (1).

Pragmatism is also required when considering whether the members of a group are mutually dependent. We are not saying that mutual dependence can never be reversed by evolutionmitochondria have been lost in some parasites of humans, and plastids have been lost multiple times $(45,63)$. Instead, our aim is to consider whether the breaking up of a group would prevent replication. Related to this point, all organisms are dependent upon other organisms to be able to reproduce, and we are considering mutual dependence relative to a defined social group. For example, we can ask whether the cells that make up a male lion are mutually dependent upon each other, even though that male is also dependent upon a female lion to mate with, zebras to eat, grass to feed those zebras and so on.

\section{The World Made Simpler}

Maynard Smith and Szathmáry offered a mixture of explanations for the different transitions, suggesting that a diversity of factors were at play (2). We suggest that both theoretical and empirical advances have provided a more unified explanation for the different major transitions. Major transitions require extreme conditions, with certain factors being either consistently important, or consistently unimportant.

First, at a very general level, the same two factors play a key role in answering all six questions that we posed: $(i)$ an ecological benefit to cooperation and (ii) a mechanism to unite the interests of different individuals. Consequently, the same ecological and evolutionary conditions can provide the answers to all six questions. For example, the combination of clonality and the right ecological benefit can remove within-group conflict (question $v$ ) and favor cooperation (questions $i$ and $i i$ ), division of labor (question iii), honest communication (question $i v$ ), and mutual dependence (question $v i$ ).

Second, at a more specific level, both theory and data suggest that how social groups form has played an analogous and fundamental role, across the different major transitions (Fig. 5). The within-species transitions to multicellularity and eusociality have occurred only when $(i)$ the social group passes through a single propagule phase (cell or singly mated female) or (ii) the social group forms by offspring staying to help their parent (subsociality) $(35-37,40)$. We suggest that transitions between species may involve similarly restrictive conditions, such as vertical transmission of clonal symbionts.

Third, the restrictive conditions required for a major transition make it easier to understand where and why major transitions have not taken place: for example, why there have been no major transitions in promiscuous cooperative breeders or multicellular groups formed by aggregation. A major transition is not driven simply by satisfying Hamilton's rule (questions $i$ and $i i$ ); it is about conflict being sufficiently eliminated that the group acts as a single maximizing agent (question $v$ ). Consequently, a lower relatedness $(r)$ cannot be made up for just with a greater ecological benefit to cooperation $(B / C)$. We would not expect major transitions with certain life histories, such as promiscuity or nonclonal aggregation $(33,35)$.

Fourth, there are striking similarities in the ecological benefits to cooperation that have driven different transitions. The eusocial insects can be divided depending upon whether the evolution of eusociality was driven by either the advantage in forming defensive groups (e.g., termites, aphids) or the efficiency benefit gained from cooperating to rear young (e.g., hymenoptera) (17). The ecological benefits to multicellularity seem to divide along analogous lines due to the benefits of forming defensive groups (e.g., algae) or to make certain traits more efficient (e.g., yeast, slime molds) $(13,64)$. Can we make similar generalizations about the transitions between species?

Fifth, within-group kin discrimination seems to have played a limited role in helping within species' major transitions. The transitions to obligate multicellularity and obligate eusociality have taken place only in clonal or monogamous populations $\left(r_{o}=r_{h}\right)$, where there is no or limited potential for kin discrimination.
Consequently, although kin discrimination can be important in facultatively multicellular species or cooperative breeders, this discrimination is only favored when there is appreciable variation in within-group relatedness (65), which also prevents major transitions. Furthermore, some cases of kin discrimination, such as haplodiploid females favoring sons over brothers, increases rather than decreases conflict, and so disfavor rather than favor major transitions. An important exception is the involvement of kin discrimination in the worker policing of the haplodiploid social insects (44).

Sixth, repression of competition seems to have played a limited role in helping within-species major transitions. The transitions to obligate multicellularity and obligate eusociality have taken place only in clonal or monogamous populations $\left(r_{o}=r_{h}\right)$, where there is no or limited competition to be suppressed. Furthermore, theory suggests that it is hard to evolve the complete repression of competition that would be required for a major transition because the marginal benefits of repressing competition will often plateau, such that an intermediate level of repression will be favored (66). As with kin discrimination, an important exception is policing in the haplodiploid social insects (44).

Considering transitions between species, the relative role of repression of competition is less clear but may also be relatively unimportant. In many cases, vertical inheritance of clonal symbionts may mean that there is no competition to repress. With horizontal transmission of symbionts, sanction mechanisms that reward cooperators and/or punish noncooperators can be favored $(23,24)$, but these sanctions may not be able to suppress competition enough for a major transition to occur.

\section{Future Directions}

We conclude by emphasizing that many of the ideas in this paper are tentative. Major theoretical issues remain unanswered, and empirical work has only begun to tackle the issues surrounding the major questions. Insights can be obtained from studies of species that haven't made major transitions, as well as those that have, or by comparing across species that have transitioned to a different extent.

First, although we have a relatively good understanding of cooperation (questions $i$ and $i i$ ), we have a much poorer understanding of group transformation (questions iii-vi). In cases where we have a good understanding, other questions arise: for example, how groups form is important, but how can we explain variation in the way in which groups form?

Second, we have a much better understanding of the withinspecies transitions than the between-species transitions. The within-species transitions have been easier to study because they have happened both more often and usually more recently than transitions such as the evolution of the eukaryote cell or the genome. Advances in genomic methodologies are allowing division of labor and mutual dependence to be much better studied in endosymbionts and organelles $(15,28,45,67)$.

Third, the applicability of the fitness-maximizing agent analogy to certain scenarios remains to be explored. For example, what are the consequences of horizontal gene transfer in bacteria, or cultural evolution (social learning) in humans? There is a lack of formal theory examining the conditions required to remove conflict and thus lead to a group acting as a single maximizing unit, especially for between-species transitions (33).

Fourth, we have focused on determining the ultimate selective forces that have favored major transitions. Mechanistic details can matter if they predispose certain species to making transitions: for example, if bacteria are able to acquire resources required by a potential host, gain entry to and proliferate in the tissues and cells of eukaryotes, and exchange symbiotic loci horizontally. Can generalizations be made about the role of predispositions within or between transitions?

Finally, we have focused on the route to making a major transition. Once a major transition has been made, evolution can lead to scenarios that reintroduce conflict into the group. For example, multicellular groups can become so large that mutation becomes an appreciable problem, and eusocial insects can evolve 
multiple mating or multiple queens $(40,60)$. In addition, mutual dependence can break down, allowing major transitions to be reversed, as has occurred with both mitochondria and plastids. Genomic reduction could lead to endosymbionts becoming so ineffective that they are lost or replaced (54). These points raise at least two more questions:

vii) How are new conflicts of interest suppressed in groups that have already made a major transition?

viii) What conditions favor the breakdown of major transitions?

As when considering questions $i-v i$, the beauty of the majortransition approach is that it facilitates the identification of general patterns. How important is repression of conflict mechanisms for

1. Bourke AFG (2011) Principles of Social Evolution (Oxford Univ Press, Oxford).

2. Maynard Smith J, Szathmáry E (1995) The Major Transitions in Evolution (Oxford Univ Press).

3. Leigh EG, Jr (1991) Genes, bees and ecosystems: The evolution of a common interest among individuals. Trends Ecol Evol 6(8):257-262.

4. Queller DC (2000) Relatedness and the fraternal major transitions. Philos Trans $R$ Soc Lond B Biol Sci 355(1403):1647-1655.

5. Michod RE (2007) Evolution of individuality during the transition from unicellular to multicellular life. Proc Natl Acad Sci USA 104(Suppl 1):8613-8618.

6. Queller DC, Strassmann JE (2009) Beyond society: The evolution of organismality. Philos Trans R Soc Lond B Biol Sci 364(1533):3143-3155.

7. Crespi BJ, Yanega D (1995) The definition of eusociality. Behav Ecol 6:109-115.

8. West SA, Griffin AS, Gardner A (2007) Social semantics: Altruism, cooperation, mutualism, strong reciprocity and group selection. J Evol Biol 20(2):415-432.

9. Ghoul M, Griffin AS, West SA (2014) Toward an evolutionary definition of cheating. Evolution 68(2):318-331.

10. Lehmann L, Keller L (2006) The evolution of cooperation and altruism: A general framework and a classification of models. J Evol Biol 19(5):1365-1376.

11. West SA, Griffin AS, Gardner A (2007) Evolutionary explanations for cooperation. Curr Biol 17(16):R661-R672.

12. Sachs JL, Mueller UG, Wilcox TP, Bull JJ (2004) The evolution of cooperation. Q Rev Biol 79(2):135-160.

13. Koschwanez JH, Foster KR, Murray AW (2013) Improved use of a public good selects for the evolution of undifferentiated multicellularity. eLife 2:e00367.

14. Moran NA (2007) Symbiosis as an adaptive process and source of phenotypic complexity. Proc Natl Acad Sci USA 104(Suppl 1):8627-8633.

15. Douglas AE (2010) The Symbiotic Habit (Princeton Univ Press, Princeton).

16. Henry LM, et al. (2013) Horizontally transmitted symbionts and host colonization of ecological niches. Curr Biol 23(17):1713-1717.

17. Queller DC, Strassmann JE (1998) Kin selection and social insects. Bioscience 48: $165-175$.

18. Hamilton WD (1964) The genetical evolution of social behaviour. I. J Theor Biol $7(1): 1-16$.

19. Bernasconi G, Strassmann JE (1999) Cooperation among unrelated individuals: The ant foundress case. Trends Ecol Evol 14(12):477-482.

20. Trivers RL (1971) The evolution of reciprocal altruism. Q Rev Biol 46:35-57.

21. Foster $K R$, Wenseleers $T$ (2006) A general model for the evolution of mutualisms. J Evol Biol 19(4):1283-1293.

22. Frank SA (1994) Kin selection and virulence in the evolution of protocells and parasites. Proc Biol Sci 258(1352):153-161.

23. West SA, Kiers ET, Simms EL, Denison RF (2002) Sanctions and mutualism stability: Why do rhizobia fix nitrogen? Proc Biol Sci 269(1492):685-694.

24. Kiers ET, Rousseau RA, West SA, Denison RF (2003) Host sanctions and the legumerhizobium mutualism. Nature 425(6953):78-81.

25. Koch EJ, Miyashiro T, McFall-Ngai MJ, Ruby EG (2014) Features governing symbiont persistence in the squid-vibrio association. Mol Ecol 23(6):1624-1634.

26. Rossetti V, Schirrmeister BE, Bernasconi MV, Bagheri HC (2010) The evolutionary path to terminal differentiation and division of labor in cyanobacteria. J Theor Biol 262(1): 23-34.

27. Gruber-Vodicka HR, et al. (2011) Paracatenula, an ancient symbiosis between thiotrophic Alphaproteobacteria and catenulid flatworms. Proc Natl Acad Sci USA 108(29):12078-12083.

28. Van Leuven JT, Meister RC, Simon C, McCutcheon JP (2014) Sympatric speciation in a bacterial endosymbiont results in two genomes with the functionality of one. Cell 158(6):1270-1280.

29. Gavrilets S (2010) Rapid transition towards the Division of Labor via evolution of developmental plasticity. PLOS Comput Biol 6(6):e1000805.

30. Maynard Smith J, Harper D (2003) Animal Signals (Oxford Univ Press, Oxford).

31. Diggle SP, Griffin AS, Campbell GS, West SA (2007) Cooperation and conflict in quorum-sensing bacterial populations. Nature 450(7168):411-414.

32. Heath-Heckman EAC, et al. (2013) Bacterial bioluminescence regulates expression of a host cryptochrome gene in the squid-Vibrio symbiosis. mBio 4(2):e00167-13.

33. Gardner A, Grafen A (2009) Capturing the superorganism: A formal theory of group adaptation. J Evol Biol 22(4):659-671.

34. West SA, Gardner A (2013) Adaptation and inclusive fitness. Curr Bio/ 23(13):R577-R584

35. Boomsma JJ (2007) Kin selection versus sexual selection: Why the ends do not meet. Curr Biol 17(16):R673-R683.

36. Boomsma JJ (2009) Lifetime monogamy and the evolution of eusociality. Philos Trans $R$ Soc Lond B Biol Sci 364(1533):3191-3207. keeping down conflicts that arise after transitions have been made, such as suppressors of selfish genes or cancer $(1,61)$ ? Do major transitions tend to break down because of new conflicts arising, or because the ecological benefits change? A new era of research focused on the commonalities in the major transitions across the tree of life is positioned to tackle these questions.

ACKNOWLEDGMENTS. We thank the organizers of the Sackler Colloquium for inviting this paper and Koos Boomsma, Andrew Bourke, Ellen Clarke, Nicholas Davies, Nicole Dubilier, Stephanie Kapsetaki, Kevin Foster, Sam Levin, Geoff Parker, Dave Queller, and Ned Ruby for useful discussion. We thank the Calleva Research Centre, Magdalen College, the European Research Council, the Natural Environment Research Council, and the Netherlands Organization for Scientific Research for funding.

37. Fisher RM, Cornwallis CK, West SA (2013) Group formation, relatedness, and the evolution of multicellularity. Curr Biol 23(12):1120-1125.

38. Grosberg RK, Strathmann RR (2007) The evolution of multicellularity: A minor major transition? Annu Rev Ecol Evol Syst 38:621-654.

39. Gilbert OM, Foster KR, Mehdiabadi NJ, Strassmann JE, Queller DC (2007) High relatedness maintains multicellular cooperation in a social amoeba by controlling cheater mutants. Proc Natl Acad Sci USA 104(21):8913-8917.

40. Hughes WO, Oldroyd BP, Beekman M, Ratnieks FL (2008) Ancestral monogamy shows kin selection is key to the evolution of eusociality. Science 320(5880):1213-1216.

41. Cornwallis CK, West SA, Davis KE, Griffin AS (2010) Promiscuity and the evolutionary transition to complex societies. Nature 466(7309):969-972.

42. Wenseleers T, Ratnieks FLW (2004) Tragedy of the commons in Melipona bees. Proc Biol Sci 271(Suppl 5):S310-S312.

43. Frank SA (1995) Mutual policing and repression of competition in the evolution of cooperative groups. Nature 377(6549):520-522.

44. Wenseleers T, Ratnieks FL (2006) Enforced altruism in insect societies. Nature 444(7115):50.

45. Keeling PJ (2013) The number, speed, and impact of plastid endosymbioses in eukaryotic evolution. Annu Rev Plant Biol 64:583-607.

46. Frank SA (1996) Host-symbiont conflict over the mixing of symbiotic lineages. Proc Biol Sci 263(1368):339-344.

47. Sachs JL, Skophammer RG, Regus JU (2011) Evolutionary transitions in bacterial symbiosis. 108(Suppl 2):10800-10807.

48. Kiers ET, et al. (2011) Reciprocal rewards stabilize cooperation in the mycorrhizal symbiosis. Science 333(6044):880-882.

49. Jandér KC, Herre EA (2010) Host sanctions and pollinator cheating in the fig tree-fig wasp mutualism. Proc Biol Sci 277(1687):1481-1488.

50. Poulsen M, Boomsma JJ (2005) Mutualistic fungi control crop diversity in fungusgrowing ants. Science 307(5710):741-744.

51. Ellers J, Kiers ET, Currie CR, McDonald BR, Visser B (2012) Ecological interactions drive evolutionary loss of traits. Ecol Lett 15(10):1071-1082.

52. Nowack ECM, Melkonian M (2010) Endosymbiotic associations within protists. Philos Trans $R$ Soc Lond B Biol Sci 365(1541):699-712.

53. McManus GB, Schoener DM, Haberlandt K (2012) Chloroplast symbiosis in a marine ciliate: Ecophysiology and the risks and rewards of hosting foreign organelles. Front Microbio/ 3:321-329.

54. McCutcheon JP, Moran NA (2012) Extreme genome reduction in symbiotic bacteria. Nat Rev Microbiol 10(1):13-26

55. Dubilier N, Bergin C, Lott C (2008) Symbiotic diversity in marine animals: The art of harnessing chemosynthesis. Nat Rev Microbio/ 6(10):725-740.

56. Shinzato C, et al. (2011) Using the Acropora digitifera genome to understand coral responses to environmental change. Nature 476(7360):320-323.

57. Gardner A, Welch JJ (2011) A formal theory of the selfish gene. J Evol Bio/ 24(8):1801-1813.

58. Gardner A (2009) Adaptation as organism design. Biol Lett 5(6):861-864.

59. Ackermann $M$, et al. (2008) Self-destructive cooperation mediated by phenotypic noise. Nature 454(7207):987-990.

60. Roze D, Michod RE (2001) Mutation, multilevel selection, and the evolution of propagule size during the origin of multicellularity. Am Nat 158(6):638-654.

61. Trivers RL (2006) Genes in Conflict (Harvard Univ Press, Cambridge, MA).

62. Sundström L, Chapuisat M, Keller L (1996) Conditional manipulation of sex ratios by ant workers: A test of kin selection theory. Science 274(5289):993-995.

63. van der Giezen M (2009) Hydrogenosomes and mitosomes: Conservation and evolution of functions. J Eukaryot Microbio/ 56(3):221-231.

64. Claessen D, Rozen DE, Kuipers OP, Søgaard-Andersen L, van Wezel GP (2014) Bacterial solutions to multicellularity: A tale of biofilms, filaments and fruiting bodies. Nat Rev Microbiol 12(2):115-124.

65. Cornwallis CK, West SA, Griffin AS (2009) Routes to indirect fitness in cooperatively breeding vertebrates: Kin discrimination and limited dispersal. J Evol Biol 22(12):2445-2457.

66. El Mouden C, West SA, Gardner A (2010) The enforcement of cooperation by policing Evolution 64(7):2139-2152.

67. Moran NA, McCutcheon JP, Nakabachi A (2008) Genomics and evolution of heritable bacterial symbionts. Annu Rev Genet 42:165-190.

68. Strassmann JE, Zhu Y, Queller DC (2000) Altruism and social cheating in the social amoeba Dictyostelium discoideum. Nature 408(6815):965-967.

69. Mehdiabadi NJ, et al. (2006) Social evolution: Kin preference in a social microbe. Nature 442(7105):881-882.

70. Sachs JL, Ehinger MO, Simms EL (2010) Origins of cheating and loss of symbiosis in wild Bradyrhizobium. J Evol Biol 23(5):1075-1089. 